\title{
The Simulation of Plasma Based Semiconductor Processing Using Block Structured Locally Refined Grids
}

\author{
Daniel Dale Wake
}

PhD Thesis

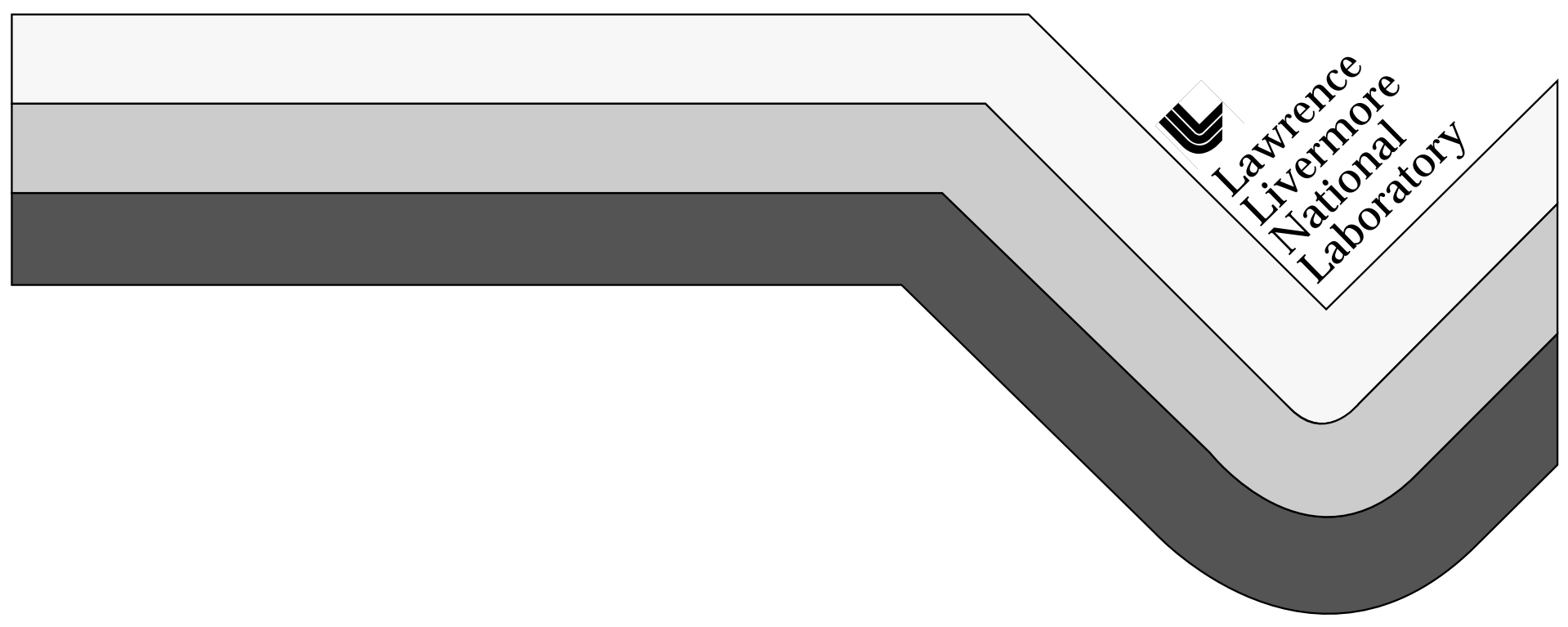




\section{DISCLAIMER}

This document was prepared as an account of work sponsored by an agency of the United States Government. Neither the United States Government nor the University of California nor any of their employees, makes any warranty, express or implied, or assumes any legal liability or responsibility for the accuracy, completeness, or usefulness of any information, apparatus, product, or process disclosed, or represents that its use would not infringe privately owned rights. Reference herein to any specific commercial product, process, or service by trade name, trademark, manufacturer, or otherwise, does not necessarily constitute or imply its endorsement, recommendation, or favoring by the United States Government or the University of California. The views and opinions of authors expressed herein do not necessarily state or reflect those of the United States Government or the University of California, and shall not be used for advertising or product endorsement purposes.

This report has been reproduced directly from the best available copy.

Available to DOE and DOE contractors from the Office of Scientific and Technical Information P.O. Box 62, Oak Ridge, TN 37831

Prices available from (615) 576-8401, FTS 626-8401

Available to the public from the National Technical Information Service

U.S. Department of Commerce 5285 Port Royal Rd., Springfield, VA 22161

Work performed under the auspices of the U.S. Department of Energy by Lawrence Livermore National Laboratory under Contract W-7405-ENG-48. 
UCRL-LR-129296

Distribution Category UC-705

\title{
The Simulation of Plasma Based Semiconductor Processing Using Block Structured Locally Refined Grids
}

\author{
Daniel Dale Wake
}

Doctor of Philosophy

Thesis

Manuscript date: January 1998

LAWRENCE LIVERMORE NATIONAL LABORATORY University of California • Livermore, California • 94551 



\title{
The Simulation of Plasma Based Semiconductor Processing Using Block Structured Locally Refined Grids.
}

\author{
by \\ Daniel Dale Wake \\ B.A. (University of California, Berkeley) 1986 \\ M.S. (University of California, Davis) 1994 \\ A dissertation submitted in partial satisfaction of the \\ requirements for the degree of \\ Doctor of Philosophy \\ in \\ Engineering - Applied Science \\ in the \\ GRADUATE DIVISION \\ of the \\ UNIVERSITY of CALIFORNIA at DAVIS
}

Committee in charge:

Garry H. Rodrigue, PhD, Chair

John S. DeGroot, PhD

Milo R. Dorr, PhD 
The Simulation of Plasma Based Semiconductor Processing Using Block

Structured Locally Refined Grids.

Copyright 1998

by

Daniel Dale Wake 


\begin{abstract}
The Simulation of Plasma Based Semiconductor Processing Using Block Structured Locally Refined Grids.

by

Daniel Dale Wake

Doctor of Philosophy in Engineering - Applied Science

University of California at Davis

Garry H. Rodrigue, PhD, Chair
\end{abstract}

This dissertation describes a numerical method for the solution of plasma fluid equations on blockstructured, locally refined grids. The plasma under consideration is typical of those used for the processing of semiconductors. The governing equations consist of a drift-diffusion model of the electrons and an isothermal model of the ions coupled by Poisson's equation. A discretization of the equations is given for a uniform spatial grid, and a time-split integration scheme is developed. The algorithm is then extended to accommodate locally refined grids. This extension involves the advancement of the discrete system on a hierarchy of levels, each of which represents a degree of refinement, together with synchronization steps to ensure consistency across levels. An analysis of the method is then conducted, both on uniform and on locally refined grids. This analysis examines the accuracy and efficiency of the method while addressing multilevel timestep selection and temporal refinement strategies for acceleration of steady state solutions. Finally, a number of applications are examined. First, the algorithm is used to perform a sheath scale simulation of a hydrogen plasma. Secondly, two problems of practical interest for manufacturing are examined. Power deposition 
effects on ion flux uniformity are examined as well as the effect of substrate RF-bias potential on energy and flux behavior. Both of these suggest the need for high resolution simulations. 


\section{Acknowledgements}

I have many people to thank for their help over these last 4 plus years.

- Foremost among these is my research advisor Milo Dorr. On an almost daily basis he has helped me in my struggle to complete this dissertation and he has provided me with a model for what a professional scientist should be.

- A special word of thanks for my academic advisor Garry Rodrigue. Garry first pointed me toward this research and has been a constant source of ideas and encouragement.

- Thanks to Phil Colella, group leader of ANAG. Much of this work represents implementations of ideas which were Phil's.

- Thanks to all the folks at CCSE in Berkeley, especially John Bell. John's scribbled notes and off-the-cuff comments may not seem like much to him, but they have been invaluable to me.

- Thanks to Peter Vitello for his many helpful discussions regarding plasma modeling. We would not have endevoured to start this work if Peter had not been so successful with his own efforts at plasma modeling.

- Thanks to Steve Ashby for treating this graduate student like any other researcher and for generously providing lots of computer time, without which I'd have lost six months.

- I would like to thank the members of my oral exam and thesis committees, John DeGroot, David Hwang, Richard Christenson and Garry Puckett.

- Thanks to the DAS staff, especially Archietta Johnson, for always knowing what forms needed signatures and when.

- Thanks to my mother, Barbara, for her constant love and for scolding me in third grade for poor scholarship. I've done much better these last 17 years of school, mom. 
- Thanks to my father, Earl, whose love of books and respect for academic achievement rubbed off on his kids.

- Thanks to my brother, Dave, the entire Khoo family and all my friends who kept me sane during this ordeal. 


\section{To Sandy}

Who encouraged me to go back to school, ignored hardship and provided moral support all along the way. As in all things, comfort and convenience were never considerations for her. It was simply something her husband wanted and therefore deserved no further thought. 


\section{Contents}

List of Figures $\quad$ vii

List of Tables $\quad \mathrm{x}$

1 Introduction 1

1.1 Overview ............................... 1

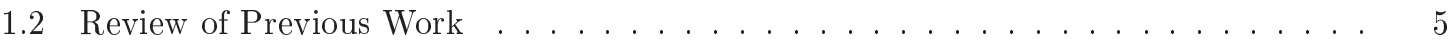

1.2.1 Simulation of Plasmas for Processing Applications . . . . . . . . . . . 5

1.2.2 Block Structured Adaptive Mesh Refinement . . . . . . . . . . . . . 7

2 Plasma Processing $\quad 10$

2.1 Plasma Reactors . . . . . . . . . . . . . . . . . . . . . . . 11

2.2 The Plasma Model . . . . . . . . . . . . . . . . . . . . . . . . . . 13

3 Single Grid Algorithm 16

3.1 Motivation .......................... 16

3.2 Time Splitting Scheme . . . . . . . . . . . . . . . . . . . . . . . . 17

3.2.1 Step 2 : Diffusive Flux Calculation . . . . . . . . . . . . . . . . . 27

3.2.2 Step 3 : Coupled Potential and Electron Drift Flux Calculation . . . . . . . 28

3.2.3 Step 4 : Internal Energy Evolution . . . . . . . . . . . . . . . . . . 29

3.2 .4 Step 5 : Final Update for Ions . . . . . . . . . . . . . . . . . . . 30

3.3 Boundary Conditions . . . . . . . . . . . . . . . . . . . . . . 31

3.4 Timestep Restrictions . . . . . . . . . . . . . . . . . . . . . . 33

4 Extension of the Algorithm to Locally Refined Grids 34

4.1 Nomenclature . . . . . . . . . . . . . . . . . . . . . . . . . 34

4.2 Solution of Poisson's Equation on a Composite Grid . . . . . . . . . . . . . . . 35

4.3 Solution of the Plasma Fluid System on a Composite Grid . . . . . . . . . . . . . . 39

4.3.1 Synchronization of the Charge and Potential . . . . . . . . . . . . . . . . 39

4.3.2 Synchonization of the Electron Internal Energy . . . . . . . . . . . . . . . . 48

4.3.3 Generalization to an Arbitrary Number of Refinement Levels . . . . . . . . . 51

4.3.4 Solution of the Composite Systems _ . . . . . . . . . . . . . . 52

5 Analysis of the Method $\quad 55$

5.1 Uniform Grid Analysis . . . . . . . . . . . . . . . . . . . . . 55

5.1 .1 Comparison to INDUCT . . . . . . . . . . . . . . . . 55

5.1 .2 Convergence of the Single Grid Algorithm . . . . . . . . . . . . . 58

5.2 Locally Refined Grid Analysis . . . . . . . . . . . . . . . . . . . . . . . 60 
5.2.1 Demonstration of Accuracy Using Locally Refined Grids . . . . . . . . . . . . 62

5.2 .2 Effect of Grid Selection on Accuracy . . . . . . . . . . . . . . . . . 65

5.2 .3 Computational Efficiency ..................... 79

5.2 .4 Multilevel Timestep Selection .................... 81

5.2 .5 Adaptivity .......................... 89

6 Applications I : Sheath Scale Hydrogen Plasma Simulation 94

6.1 Ingold Model . . . . . . . . . . . . . . . . . . . . . . . . . . . . . . . . . . . . . . . . . . .

6.2 Comparison with APM . . . . . . . . . . . . . . . . . . . . 97

7 Applications II : Simulations of Inductively Coupled Plasma Reactors 101

7.1 Power Deposition Profile Effects . . . . . . . . . . . . . . . . . . . . . 101

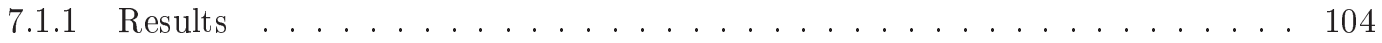

7.2 Wafer Biasing Effects . . . . . . . . . . . . . . . . . . . . . 104

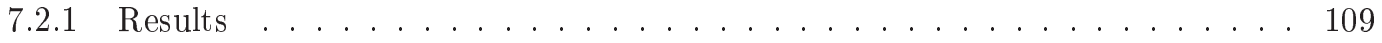

8 Conclusion

115

A Linearized Stability Analysis of the Coupled Electron/Potential Update Scheme117

A.1 Introduction . . . . . . . . . . . . . . . . . . . . 117

A.2 Linearized Model Equations . . . . . . . . . . . . . . . . . . . . . 118

A.3 APM Algorithm ............................. 119

A.3.1 Fourier Analysis . . . . . . . . . . . . . . . . . . . 121

A.4 Simplified Systems . . . . . . . . . . . . . . . . . . . . . 122

A.4.1 Cold Electrons . . . . . . . . . . . . . . . . . . . . . . . . 122

A.4.2 Zero Mean Field . . . . . . . . . . . . . . . . . . . . 124

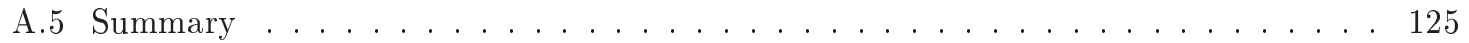

B Derivation of the Fluid Equations from the Boltzmann Equation 126

B.1 Velocity Distribution . . . . . . . . . . . . . . . . . 126

B.2 Moments ............................ 127

Bibliography $r 132$ 


\section{List of Figures}

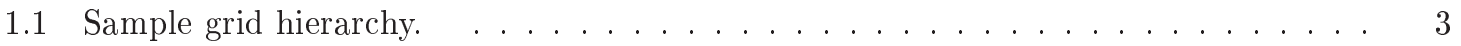

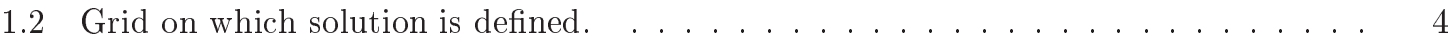

2.1 Generic inductively coupled reactor. . . . . . . . . . . . . . . . . . . 12

3.1 Calculation of left and right states for Riemann problems for edges normal to the $x$ direction. Arrows indicate position for state predictions relative to base of Taylor

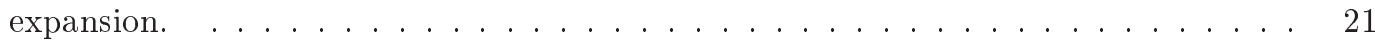

3.2 Calculation of edge electron densities based on drift velocity direction. . . . . . . 28

4.1 Domain definitions for the composite grid. The top figure shows a "side" view of the fine and coarse grids separately, while the bottom figure shows a view from "above" of the composite grid. The composite grid is the union of the fine grid and that portion of the coarse grid not covered by the fine grid. . . . . . . . . . . . .

4.2 (a) At a physical boundary, interior and boundary values (large o's) are used to extrapolate to the ghost cell (०); the ghost value and the other interior values (•'s) are used to construct the Laplacian at (a). (b) Locations of coarse grid boundary conditions (large $\bullet$ ), tangentially interpolated values $(\circ)$, fine grid cells $(\bullet)$, and ghost cells (small $\triangle$ 's and small $\square$ 's). (c) Domain of dependence $(\bullet$ 's and large $\bullet$ 's) of the Laplacian at a fine cell (large $\circ$ ) adjacent to the coarse/fine interface. . . . . . . . . 38

4.3 Multilevel advance and composite synchronization schedule. . . . . . . . . . . . . . 52

5.1 Potential profiles for APM and INDUCT test case. . . . . . . . . . . 56

5.2 Electron density profiles for APM and INDUCT test case. . . . . . . . . . . 57

5.3 Ion flux profiles for APM and INDUCT test case. . . . . . . . . . . . . . 57

5.4 Electron temperature profiles for APM and INDUCT test case. . . . . . . . . 58

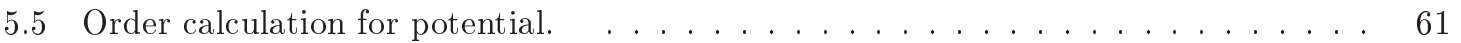

5.6 Order calculation for electron density. . . . . . . . . . . . . . . . . . . . 61

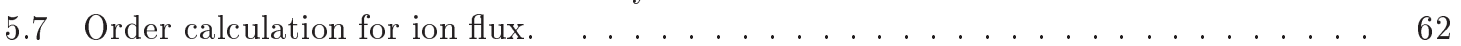

5.8 Potential profile for locally refined, fine and coarse grid calculations. . . . . . . . . 63

5.9 Ion flux profile for locally refined, fine and coarse grid calculations. . . . . . . . . 63

5.10 Electron density profile for locally refined, fine and coarse grid calculations. . . . . . 64

5.11 Electron temperature profile for locally refined, fine and coarse grid calculations. . . 64

5.12 Locally refined, uniform fine and uniform coarse grids used to generate results shown in Figures 5.8 through $5.11 \ldots \ldots \ldots \ldots$. . . . . . . . . . . . . . 65

5.13 Grids used in multilevel study of solution dependence on number of levels and refine-

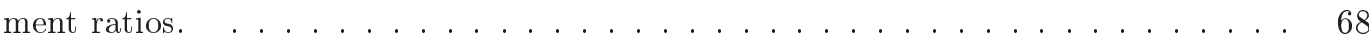


5.14 Relative error in the potential for different grid structures. The first two numbers give the number of cells in the $x$ and $y$ directions, respectively. Subsequent numbers give refinement ratios for finer levels (if they exist). . . . . . . . . . . . .

5.15 Relative error in the net charge for different grid structures. The first two numbers give the number of cells in the $x$ and $y$ directions, respectively. Subsequent numbers give refinement ratios for finer levels (if they exist). . . . . . . . . . . . .

5.16 Relative error in the electron density for different grid structures. The first two numbers give the number of cells in the $x$ and $y$ directions, respectively. Subsequent numbers give refinement ratios for finer levels (if they exist). . . . . . . . . .

5.17 Relative error in the ion density for different grid structures. The first two numbers give the number of cells in the $x$ and $y$ directions, respectively. Subsequent numbers give refinement ratios for finer levels (if they exist). . . . . . . . . . . . .

5.18 Relative error in the electron temperature for different grid structures. The first two numbers give the number of cells in the $x$ and $y$ directions, respectively. Subsequent numbers give refinement ratios for finer levels (if they exist). . . . . . . . . . .

5.19 Relative error in the ion flux for different grid structures. The first two numbers give the number of cells in the $x$ and $y$ directions, respectively. Subsequent numbers give refinement ratios for finer levels (if they exist). . . . . . . . . . . . . .

5.20 Grids used in two level accuracy study of dependence on the number of cells refined.

5.21 Relative error in the potential at $10 \mu$ s for different refined regions. The labels refer to the number of cells refined near the boundary. . . . . . . . . . . . . .

5.22 Relative error in the net charge at $10 \mu$ s for different refined regions. The labels refer to the number of cells refined near the boundary. . . . . . . . . . . .

5.23 Relative error in the electron density at $10 \mu$ s for different refined regions. The labels refer to the number of cells refined near the boundary. . . . . . . . . . . . .

5.24 Relative error in the ion density at $10 \mu \mathrm{s}$ for different refined regions. The labels refer to the number of cells refined near the boundary. . . . . . . . . . . . .

5.25 Relative error in the electron temperature at $10 \mu$ s for different refined regions. The labels refer to the number of cells refined near the boundary. . . . . . . . . . . . .

5.26 Relative error in the ion flux at $10 \mu \mathrm{s}$ for different refined regions. The labels refer to the number of cells refined near the boundary. . . . . . . . . . . . .

5.27 Relative error in the potential at $50 \mu \mathrm{s}$ for different refined regions. The labels refer to the number of cells refined near the boundary. . . . . . . . . . . . .

5.28 Relative error in the net charge at $50 \mu$ s for different refined regions. The labels refer to the number of cells refined near the boundary. . . . . . . . . . . . .

5.29 Relative error in the electron density at $50 \mu$ s for different refined regions. The labels refer to the number of cells refined near the boundary. . . . . . . . . . . .

5.30 Relative error in the ion density at $50 \mu$ s for different refined regions. The labels refer to the number of cells refined near the boundary. . . . . . . . . . . . . .

5.31 Relative error in the electron temperature at $50 \mu \mathrm{s}$ for different refined regions. The labels refer to the number of cells refined near the boundary. . . . . . . . . . .

5.32 Relative error in the ion flux at $50 \mu$ s for different refined regions. The labels refer to the number of cells refined near the boundary. . . . . . . . . . . . . 78

5.33 Ratio of CPU times for uniform and locally refined grid calculations. . . . . . . . 82

5.34 Ratio of memory requirements for uniform and locally refined grid calculations. . . 82

5.35 Sample grid used to calculate time and storage savings shown in Figure 5.33. This grid corresponds to the case with refinement ratios of 2 and 4 refined levels. . . . . 83

5.36 Errors for subcycle study at $10 \mu \mathrm{s} . \ldots \ldots \ldots \ldots \ldots$. . . . . . . . . 86

5.37 Errors for subcycle study at $50 \mu s . \ldots \ldots \ldots \ldots \ldots \ldots \ldots$

5.38 Errors for subcycle study at $100 \mu s . \ldots \ldots \ldots \ldots$. . . . . . . . . 88 
5.39 Order of grid advances to evolve a two level problem with a refinement ratio of 4 to a new, common time. The upper intervals represent the fine grid and lower interval represents the coarse grid. . . . . . . . . . . . . . . . . . 90

6.1 Grid structure for hydrogen sheath calculation. . . . . . . . . . . . . . . . . 95

6.2 Comparison of APM and Ingold sheath model for potential. . . . . . . . . . . . . . 98

6.3 Comparison of APM and Ingold sheath model for flux. . . . . . . . . . . . . . . . 99

6.4 Comparison of APM and Ingold sheath model for electron and ion densities. . . . . . 100

7.1 Computational domain for the locally refined calculations used to study power deposition effects. . . . . . . . . . . . . . . . . . . . . . . . 102

7.2 Coil positions and power density contours for power deposition study (uniform power density case not shown) . . . . . . . . . . . . . . . . . . . . 103

7.3 Coarse, uniform solution for coil placements 2 and $3 \ldots \ldots \ldots \ldots$

7.4 Wafer flux uniformity error for refined and uniform grids. . . . . . . . . . . 106

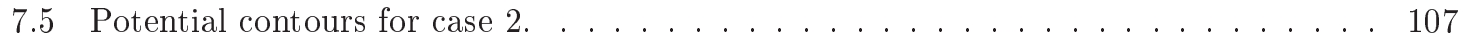

7.6 Electron temperature contours for case $2 \ldots \ldots \ldots \ldots \ldots \ldots$

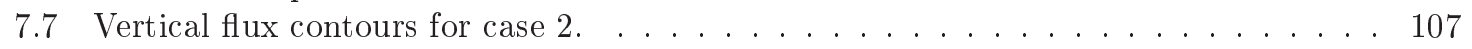

7.8 Potential contours for case $3 . \ldots \ldots \ldots \ldots$. . . . . . . . . . . . . 108

7.9 Electron temperature contours for case $3 \ldots \ldots \ldots \ldots$. . . . . . . . . 108

7.10 Vertical flux contours for case $3 . \ldots \ldots$. . . . . . . . . . . . . . 108

7.11 Time averaged potentials for zero bias (above) and 20 volt RF bias (below) cases. . . 110

7.12 Time averaged electron density for zero bias (above) and 20 volt RF bias (below) cases.111

7.13 Computational domain used in the locally refined calculations as part of the wafer

bias study. . . . . . . . . . . . . . . . . . . . . . . . 112

7.14 Ion normal flux for locally refined grid calculations. . . . . . . . . . . . . . . . 112

7.15 Ion energy per particle for locally refined grid calculations. . . . . . . . . . . . . 113

7.16 Ion normal flux for uniform and locally refined grid calculations. . . . . . . . . . . 113

7.17 Ion energy per particle for uniform and locally refined grid calculations. . . . . . . . 114 


\section{List of Tables}

2.1 Variables used in the governing equations. . . . . . . . . . . . . . .

5.1 Errors relative to high resolution uniform grid calculation for selected quantities at $10 \mu s$ (values are percent unless otherwise stated). . . . . . . . . . . . .

5.2 Errors relative to high resolution uniform grid calculation for selected quantities at $10 \mu s$ (values are percent unless otherwise stated). . . . . . . . . . . . . . 79

5.3 Comparison of computational complexity for different limiting timesteps. . . . . . 80

5.4 Timings for different subcycle strategies. . . . . . . . . . . . . . . . . . . 85

5.5 Comparison of timings for applications calculations using refinement in time with estimates for equivalent static grid calculations. Times are in seconds and are cumulative. 93 



\section{Chapter 1}

\section{Introduction}

This dissertation describes a new numerical method for the simulation of certain plasmas relevant to semiconductor manufacturing. The method is described, an analysis of its performance is undertaken and the method is used to conduct a number of simulations of interest for plasma based semiconductor manufacturing. In addition to an advance to the state of plasma simulation, this work represents an extension of the block structured Adaptive Mesh Refinement (AMR) technique to a new discipline : plasma simulation.

\subsection{Overview}

Many of the process steps performed in the manufacture of very large scale integrated (VLSI) circuits involve plasmas. Inductively coupled plasma (ICP) reactors represent one type of processing tool that utilizes high-density, low-pressure plasmas to satisfy the demanding process criteria resulting from the desire to create increasingly smaller device features on large wafers. Computational simulators of ICP reactors can help equipment manufacturers and process engineers understand the complex relationships among reactor and plasma parameters $(e . g .$, reactor geometry, RF power, gas pressure, gas composition, electrode bias, densities, velocities, etc.) and process 
performance (e.g., etch rate, anisotropy, uniformity, selectivity, damage, etc.).

One of the primary difficulties in the development of computational models of plasma processes is the need to address problems associated with a wide range of temporal and spatial scales. The high-density, low-pressure plasmas employed in ICP reactors are particularly challenging in this regard. Here, the difference between the dielectric relaxation time of the plasma and the reactor transit time for an ion can be many orders of magnitude. Moreover, due to the small Debye length of such plasmas, the sheath and presheath regions over which much of the potential drop occurs can be three or four orders of magnitude smaller than the reactor dimensions. Numerical methods must therefore be designed to accommodate large scale variations.

In this thesis, an algorithm is presented for the solution of a system of plasma fluid equations on locally refined grids. We first focus our attention on the discretization and solution of the fluid system on a grid structure with multiple levels of resolution that are prescribed a priori. This is later extended to grids which change with time. Our approach represents an extension of methodologies developed for neutral flows using block structured Adaptive Mesh Refinement (AMR). The term "adaptive mesh refinement" is rather general, but we will use this term with the specific method developed by Berger and Oliger [10] in mind. This method uses a nested hierarchy of unions of uniform rectangular grids with different resolutions. Figures 1.2 and 1.1 depict this grid structure for a sample problem. In this example we have 3 levels of computational grids which are shown in Figure 1.1, but only consider the solution on a composite grid shown in Figure 1.2. These issues are discussed in detail in Chapter 4. We mention this now in order to contrast these grid structures with those used in other methods such as unstructured grid or structured methods without this grid hierarchy. We consider a static, locally refined grid structure to be a special case of an AMR grid. The motivation for this point of view is that in most respects our algorithm corresponds to the methodologies of the AMR of Berger and Oliger. The major distinction being that we may choose to evolve our grids in time, but it is not an essential part of our algorithm. However, the efficient solution of the equations on locally refined grids is essential to our approach. We will in general 


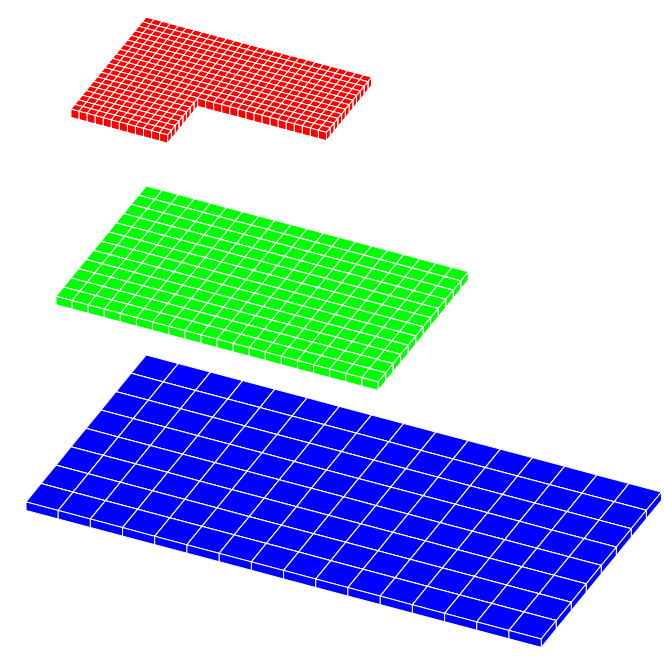

Figure 1.1: Sample grid hierarchy.

use the term "locally refined" for cases where the grids are not moving in time or where the grid movement is not important. We will generally reserve the "adaptive" term for the more general case of grids which evolve with the solution.

Chapter 1 includes this introduction and a review of previous work in the field. This includes computational modeling of plasma based manufacturing equipment and a broad overview of previous work using block structured, adaptive mesh refinement.

Chapter 2 introduces the application and describes the governing equations for our model. A general set of fluid equations is given and a simplified version is developed. The simplifications are discussed and their physical basis is explained.

Chapter 3 describes the algorithm for evolving the solution on a single uniform Cartesian grid. A time splitting scheme is explained and the details of its different components are given. Boundary conditions are described and timestep restrictions are given.

Chapter 4 extends the single grid algorithm to a hierarchy of locally refined grids. The approach for Poisson's equation with two different spatial resolutions is first described and then 


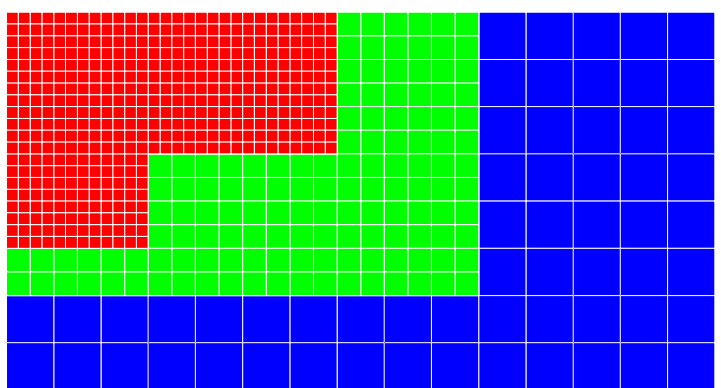

Figure 1.2: Grid on which solution is defined.

generalized for the plasma model. The method is then generalized to an arbitrary number of levels and a multilevel composite synchronization procedure is outlined.

Chapter 5 is devoted to an analysis of the algorithm. A comparison to the INDUCT [63] code is made and convergence studies are presented. We then address the issues of accuracy and efficiency of the method on a hierarchy of locally refined grids. The effects of various grids on accuracy and runtime are shown and the effects of different subcycling schedules are examined. Criteria for refinement are reviewed and we conclude by demonstrating acceleration to steady state through refinement in time.

Chapter 6 contains the results of the methods application to a sheath scale simulation of a hydrogen plasma. An analytic sheath model appropriate to our plasma model is described. The APM solution in the sheath region is shown and compared to the analytic case.

Chapter 7 applies the code to two different problems of engineering interest. First, the questions of RF coil placement and power deposition effects are studied. Second, the effect of a sinusoidal potential bias on the wafer is examined for its effect on ion flux and energy distributions. 
Chapter 8 is a conclusion.

\subsection{Review of Previous Work}

Plasma physics is a mature, well developed subject and computer simulations of plasmas date back to the Second World War. Diverse applications such as fusion energy, space physics and power electronics have driven a great deal of work on computational plasma models. An interesting history of fusion energy can be found in Herman [30] and a bibliography of early computer simulation work was compiled by Van Duzer and Birdsall [59]. Due to the large body of research on computational plasma physics, this review will be limited to simulation work related to plasma based semiconductor manufacturing.

The development of Adaptive Mesh Refinement will be reviewed along with a survey of its many applications. The term "AMR" is rather generic. It can be used to describe any type of algorithm where the grid structure is changing in time. We are specifically refering to algorithms using block structured, Cartesian grids with variable resolution of the type of Berger and Oliger.

\subsubsection{Simulation of Plasmas for Processing Applications}

Though plasma simulations for processing applications have been performed for a number of years, the recent move toward smaller feature sizes and larger wafers (along with the growth of the trillion dollar semiconductor industry) have driven tremendous growth in simulation work. Universities, national laboratories and industry have all participated.

The most extensive effort has been at the University of Illinois under Kushner. They have developed a modular simulation environment using the Hybrid Plasma Equipment Model (HPEM) [45]. The approach used in HPEM is to include a number of different physics modules (electromagnetic, chemistry, fluid, monte carlo collisions, etc.), each communicating with the other modules but acting autonomously, until all modules converge to a common solution [61]. This has the advantage 
of allowing the inclusion of different physical modules, which is clear from the number and variety of investigations which have been conducted with the code suite $[31,32,38,48,50,55,60]$. This iterative approach has been used by others as well [41]. One disadvantage of this methodology is the difficulty examining transient behavior, although some effort has been made in this regard [55].

One of the most widely used simulation tools is the INDUCT $[52,53]$ code. The code is two-dimensional, axi-symmetric and uses a two-fluid plasma model. INDUCT includes electron heating with a self-consistent treatment of the inductive fields generated by the RF coils, though the magnetoinductive fields in the fluid are not included. INDUCT includes a number of other features of practical interest to the semiconductor manufacturing community including neutral flow, multiple ion species, variable ion temperature, and complex chemistry. It has been benchmarked against experiment $[12,65]$ and has been used extensively within the semiconductor industry. Work at the University of California at Berkeley has extended the code to include complex chemistry and the effect of these reactions have been investigated with the code [13].

The difficult problem of RF coil/plasma interaction has been addressed through computer simulation. This work has focused on the inclusion of electromagnetic fields within fluid models. The FMRZ code [23] is similar to INDUCT in that it is two-dimensional and axi-symmetric, but it also includes a magnetoinductive coupling between the plasma and RF source in the third $(\Theta)$ dimension. A similar approach has been used within HPEM [38].

The desire to accurately model reactors in the low pressure regime has motivated simulation work using particle methods. The first particle simulations of ICP reactors used the Direct Simulation Monte Carlo (DSMC) technique [24]. One difference of this implementation of DSMC from standard Particle in Cell (PIC) is the decoupling of the fields and particles. An electron fluid and Poisson solution were self-consistently calculated and then used for the particle transport. The PIC method has also been used extensively at the University of California at Berkeley for plasma simulation and has recently been applied to plasma processing simulation [57]. The DADIPIC method is fully kinetic, utilizing a PIC method for both the ions and electrons, while self-consistently solving 
a reduced set of Maxwell's equations for magnetoinductive fields [27].

Commercial software is only now beginning to appear. An example is an unstructured grid code developed by the CFD corporation, which has been used in ICP investigations [67].

\subsubsection{Block Structured Adaptive Mesh Refinement}

The first description of the block structured AMR technique was given by Berger and Oliger in 1984 [10]. In this work hyperbolic partial differential equations, in one and two dimensions, were solved using finite difference methods on a hierarchy of regular Cartesian grids of variable resolution. The characteristic recursive nature of AMR was first developed here along with other common features such as Richardson extrapolation for error estimation and cell tagging and clustering algorithms. Other early work also focused on one and two dimensional systems of hyperbolic equations, especially those arising from gas dynamics and shock physics [9, 29]. This work on hyperbolic systems was later extended to three dimensions and more sophisticated gridding algorithms were developed [6].

The generalization of AMR for systems other than those which are purely hyperbolic has generated a number of technical challenges. Elliptic, parabolic and mixed systems lack the real characteristic structure and transit time isolation which make hyperbolic AMR straightforward in comparison. Many of these difficulties have to do with the need to solve linear systems and to satisfy elliptic "matching conditions". The multigrid method of Brandt [11] uses a hierarchy of grids with different resolutions, each sharing the same physical domain, in order to solve linear systems. The motivation for using different grids in this case pertains to mode damping properties of smoothing operators defined on different grids. This contrasts with AMR grids in which the reduction in local truncation error drives the use of variable resolution grids. Both techniques, however, use similar grid structures (if for different reasons). This being the case, and since multigrid is among the fastest and most efficient methods for solving linear systems in any case, it is the most widely used method for solving linear problems in AMR applications. The satisfaction of elliptic matching conditions 
for Poisson's equation has been described in [43]. Much of the recent work with AMR has been on non-hyperbolic problems. Some examples are incompressible flows, for which adaptive projection methods have been developed [2], combustion simulation [47], and radiation transport [36].

One of the only applications of AMR to electromagnetic simulations is the work of Steiner et. al. [51] in which an adaptive magnetohydrodynamic (MHD) model was used to efficiently model solar magnetoconvection.

While the vast majority of work with AMR has involved continuum models, some researchers have used particle methods. Almgren [1, 4] used AMR and a discrete set of particles "carrying vorticity" to simulate unsteady, incompressible flows. Current work involves the use of Direct Monte Carlo Simulation (DSMC) on AMR grids to capture kinetic effects [26].

While the computational efficiency of block structured AMR ( as compared to unstructured grid methods) seems clear, the difficulty in including complex geometrical objects within flow fields has been a shortcoming of the method. Consequently, considerable work has been undertaken in order to include geometric capability within the AMR framework. The first progress on this matter was due to Chern and Colella [14]. In this work a fluid redistribution scheme was developed for hyperbolic conservation laws in which internal non-orthogonal boundaries could be included. This method prevents Courant timestep limitations due to small "cut" cells, intersected by the complex body under consideration. The penalty for this capability is the lowering of the local truncation error from second to first order. These ideas have been used in the development of three dimensional AMR algorithms for compressible [46] and incompressible [3] flows. A different approach due to Chesshire and Henshaw utilizes composite overlapping grids. The grids are logically rectangular but are mapped to curvilinear coordinates. Though not strictly an AMR method of the Berger and Oliger type, many of the issues involved in solving elliptic problems with internal boundaries were addressed by workers at Boeing in solving the potential equation of aerodynamics [68].

Sophisticated software libraries have been developed for implementing AMR algorithms $[49,66]$ and domain decomposition strategies for parallel computer architectures have been developed 
$[7,8]$. 


\section{Chapter 2}

\section{Plasma Processing}

The use of plasmas for the manufacturing of semiconductors began in the late 1960's and early 1970's. Chemical Vapor Deposition (CVD) and etching were early applications. One motivation for plasma use in etching was the replacement of harsh chemicals with less volatile gases. These practical engineering and environmental concerns soon gave way to more pressing issues. As semiconductor components decreased in size, the isotropy inherent in chemical etching eliminated its usefulness for many etching applications. The anisotropic etching characteristic of plasmas became the driving force behind the development of plasma based semiconductor manufacturing equipment [42].

A detailed discussion of etching is beyond the scope of this work. However a few basic concepts are useful in order to understand how this work fits in to the application as a whole. The mechanisms for etching may be divided into three types : simple chemical etching, sputtering and ion enhanced (also known as reactive ion) etching. Sputtering removes material by brute force. The kinetic energy of incident ions is responsible for the removal of material from a substrate. In chemical etching a feed gas reacts with the wafer surface and removes material. In ion enhanced etching, impinging ions and a reactive gas combine to anisotropically etch at a rate much faster than either chemical etching or sputtering alone would provide. The exact mechanisms responsible for 
the high etch rates and anisotropy are not well understood. One theory is that the impinging ions deliver kinetic energy wich break chemical bonds or enhances reaction rates in some way. This would allow chemical etching to take place more quickly and anisotropically. Another theory is that the ions serve to keep the surface free of an oxide layer. This oxide layer would otherwise form a barrier between the etchant gas and the substrate. In any event, an understanding of incident ion fluxes on wafer surfaces is clearly a necessary component for a quantitative understanding of sputtering or ion enhanced etching.

\subsection{Plasma Reactors}

Various types of plasma reactors have been developed for etching applications. Some use magnetic field confinement, others such as those of the Electron Cyclotron Resonance (ECR) or helicon type take advantage of wave/plasma resonances for efficient coupling of external power. Many plasma reactors utilize RF heating of the plasma and do not typically involve magnetic confinement. Reactors using RF heating are commonly divided into two types : capacitively coupled and inductively coupled. In capacitively coupled plasmas, the electric fields which are responsible for Joule heating are normal to the electrode surfaces (much like the geometry of a parallel plate capacitor). These devices are conceptual simple, but have the drawback that the power deposition and particle energies at the wafer are tightly coupled. In inductively coupled systems, the fields responsible for plasma heating are parallel to the etching surface. If an independent RF bias is applied to the wafer, independent control of ion flux and RF power deposition is possible. In this thesis, the reactors we consider are of the inductively coupled type.

The idea of using inductive coupling for a plasma source to assist manufacturing processes was developed in the late 1980's and early 1990's [19, 44]. Figure 2.1 shows a generic ICP reactor and the region we consider in our model. Most are approximately cylindrically symmetric, though not all. Many of the components of such devices are not shown including pumping ports, wafer cooling and clamping apparatus, etc. The details of different reactors vary among manufacturers 


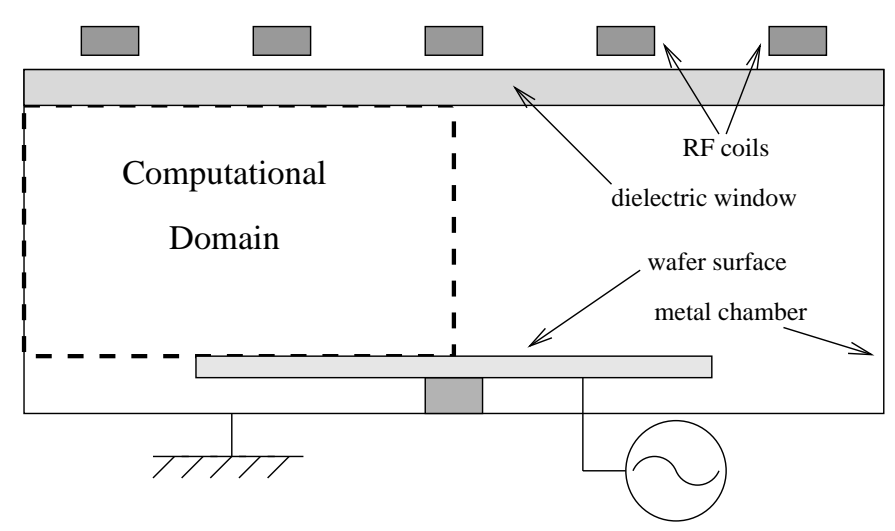

Figure 2.1: Generic inductively coupled reactor.

and models. We do not consider a specific make or model.

The driving coils provide one of the most important controls over the plasma character. Along with the neutral gas density, the input power provides the most important control over plasma density. Different coil placements effect the plasma density and temperature. These in turn are coupled in a complex way to ion fluxes on the wafer surface. The non-linear coupling of the plasma to the coils and driving circuit makes the problem very difficult to understand, though experimental and computational work has been done in this regard [22, 34, 35, 53].

While some discussions of coil/plasma interactions are found in the literature, an extensive literature search found little regarding wafer biasing effects. Some frequency effects have been investigated [42] and the qualitative relationships between the magnitude of the bias and particle flux are known [62].

Neutral gas densities in ICP reactors vary, but the trend is toward lower pressure systems. Higher densities (and hence pressures) provide larger particle fluxes, but they also decrease the mean free path for ion-neutral collisions. Ion-neutral collisions can alter the otherwise normal paths near the wafer surface. Such deviations from the normal direction decrease the desired anisotropy. An examination of the literature suggests that many ICP systems run in the 1-100 mTorr range.

A variety of gases are used in plasma sources and typically different mixtures of gases 
are used for different etching applications. Molecules containing fluorine such as $\mathrm{CF}_{4}$ and $\mathrm{SF}_{6}$ are commonly used for silicon and polychrystalline silicon etching, while chlorine containing molecules such as $\mathrm{CCl}_{4}$ and $\mathrm{SiCl}_{4}$ are used for aluminum etching. Diatomic oxygen is often used for removal of photoresistive material. While argon is used in some gas mixtures, it is not an important gas for etching. It has been widely used in experimental investigations, however. In that complex chemistry is beyond the scope of this work and that argon discharges are well understood, we have almost exclusively considered argon plasmas in this work.

\subsection{The Plasma Model}

The governing equations for a plasma fluid model are based on Maxwell's equations along with moments of the Boltzmann equation. The plasma components are assumed to have a drifting, near-Maxwellian velocity distribution. The infinite set of moment equations is truncated and we retain only the first three. These moments correspond to the conservation of mass, momentum and energy for each species. This is the starting point for our model. The derivation of the fluid equations from the Boltzmann equation is given in Appendix B and a detailed development can be found in [28].

We wish to apply these equations to a two-species plasma of electrons and singly ionized ions. We first make some additional approximations. For the electrons, we make the drift-diffusion approximation for the electron directed velocity. This approximation is sometimes referred to as the zero mass approximation, but actually it is just the elimination of inertial terms which are negligible provided that phenomena on the time scale of plasma oscillations are not resolved. The result is the replacement of the electron momentum equation with an equation of state for the electron flux determined from the balance of forces

$$
n_{e} u_{e}=, d_{\text {dift }}+, \text { diff }
$$


where

$$
\begin{gathered}
\text { drift }=-\mu n_{e} E,, \text { diff }=-\eta \nabla\left(n_{e} k T_{e}\right) \\
\mu=e / m_{e} \nu_{e n}, \eta=1 / m_{e} \nu_{e n}
\end{gathered}
$$

where, drift is the drift flux and, diff is the diffusive flux. These and the remaining variables are listed in Table (2.1). For convenience, we use the electron continuity equation and (2.1) to replace the electron energy equation by an equation for the internal energy $\frac{3}{2} n_{e} k T_{e}$.

We assume that the ions are isothermal and have the same temperature as a uniform isothermal background gas. We therefore do not need to retain the ion energy equation. The assumption of isothermal ions is made to simplify the model, but is not essential to the algorithm and implementation described here.

The coupling of external power to the plasma is via a deposition source term in the electron energy equation. We assume that this source term is known a priori rather than include a full electromagnetic model. For ICP applications, the high thermal conductivity of the plasma along with the skin depth limited penetration of electromagnetic fields make the decoupling of the source and plasma a reasonable approximation provided the region of interest is more than a few skin depths away from the coils (typically a few centimeters). With this simplification and the absence of external magnets, we may neglect magnetic fields. The electromagnetic behavior is then described by a time-varying Poisson's equation.

With these modifications, we are left with 6 time-dependent scalar equations in two spatial dimensions. They are the electron continuity and internal energy equations

$$
\begin{aligned}
\frac{\partial n_{e}}{\partial t}+\nabla \cdot\left(u_{e} n_{e}\right)= & \nu_{i z} n_{e} \\
\frac{\partial\left(n_{e} \frac{3}{2} k T_{e}\right)}{\partial t}+\nabla \cdot\left(n_{e} \frac{5}{2} k T_{e} u_{e}\right)= & -e n_{e} u_{e} \cdot E+\nabla \cdot\left(\frac{5 \eta}{2}\left(n_{e} k T_{e}\right) \nabla k T_{e}\right) \\
& +P_{\text {ind }}-\left(\nu_{i z} \epsilon_{i z}+\nu_{\text {elas }} \epsilon_{\text {elas }}+\frac{1}{2} m_{e} u_{e} \cdot u_{e} \nu_{i z}\right) n_{e}
\end{aligned}
$$

the ion continuity and momentum equations

$$
\frac{\partial n_{i}}{\partial t}+\nabla \cdot\left(u_{i} n_{i}\right)=\nu_{i z} n_{e}
$$




\begin{tabular}{|c|l|}
\hline$n_{e}, n_{i}$ & densities \\
$u_{e}, u_{i}$ & velocities \\
$\phi$ & potential \\
$E$ & electric field \\
$T_{e}, T_{i}$ & temperatures \\
$P_{i n d}$ & input power density \\
$,_{i},,_{e}$ & particle flux \\
$d_{\text {drft }},,_{\text {diff }}$ & electron drift and diffusive flux \\
$\mu_{,} \eta$ & transport coefficients \\
$m_{e}, m_{i}$ & masses \\
$q_{e}, q_{i}$ & charges \\
$\nu_{i z}, \nu_{e l a s}$ & ionization and elastic collision frequencies \\
$\epsilon_{i z}, \epsilon_{\text {elas }}$ & energy loss for ionization and elastic collision \\
$\nu_{e n}, \nu_{i n}$ & particle-neutral collision frequencies \\
$e$ & elementary charge \\
$\epsilon_{0}$ & permittivity of free space \\
$k$ & Boltzmann's constant \\
\hline
\end{tabular}

Table 2.1: Variables used in the governing equations.

$$
\frac{\partial\left(n_{i} u_{i}\right)}{\partial t}+\nabla \cdot\left(n_{i} u_{i} u_{i}\right)+\frac{k T_{i}}{m_{i}} \nabla n_{i}-\frac{q_{i} n_{i}}{m_{i}} E=u_{i}\left(\nu_{i z} n_{e}-\nu_{i N} n_{i}\right)
$$

and Poisson's equation

$$
\frac{\epsilon_{0}}{e} \nabla^{2} \phi=n_{e}-n_{i}, \quad E=-\nabla \phi
$$




\section{Chapter 3}

\section{Single Grid Algorithm}

\subsection{Motivation}

Before describing the single grid algorithm, we consider the plasma characteristics and mathematical difficulties which affect the choice of an algorithm.

The relatively slow motion of the ions allows some flexibility in the manner in which they are advanced. We do, however, want to retain high spatial accuracy since the ion flux is an important quantity for processing applications. If we explicitly treat the low order terms in (2.6) and (2.7), the ion equations form a nonlinear, vector conservation law. Such equations are well understood and stable, second-order advection schemes have been developed to solve them [17].

The electron density and potential update is the most challenging part of the algorithm. Solving Poisson's equation with explicitly advected electrons would force the use of a prohibitively small timestep due to the dielectric relaxation limit [5, 52]. A tight coupling of the electron density and potential is assured by including the drift flux with the Laplacian operator, forming a modified Poisson equation. This implicit treatment eliminates problems associated with dielectric relaxation timestep constraint, but does not result in an unconditionally stable algorithm.

The drift flux within the modified Laplacian operator depends on the electron density as 
well as the potential. The diffusive flux term appearing on the right hand side of the modified Poisson equation depends on the electron density. To avoid simultaneously solving for the electron density and the potential, we approximate the density used for these terms. For the drift flux, the electron density is approximated at $t^{n+1 / 2}$ on cell edges using a slope-limited, upwind Taylor-series expansion relative to the lagged drift velocity. This density approximation is denoted by $n_{e}^{n+1 / 2}$. For the diffusive flux, we implicitly solve for an electron density approximation $\widehat{n}_{e}^{n+1}$ at the new time. Because the maximum electron thermal velocity is much less than the drift velocity, which determines the timestep, the temperature variation is slow and the temperature can be lagged in the diffusive flux term. The electron flux term is then approximated by

$$
n_{e} u_{e} \approx, \text { drift }+, \text { diff }
$$

where

$$
\text { , drift } \equiv-\mu^{n} n_{e}^{n+1 / 2} E^{n+1}, \text { diff } \equiv-\eta^{n} \nabla \widehat{n}_{e}^{n+1} k T_{e}^{n}
$$

The internal energy equation involves a stiff heat flux term and is treated implicitly as well.

\subsection{Time Splitting Scheme}

The time splitting scheme contains 5 steps which are summarized here and detailed below.

1. Explicitly calculate the flux divergence needed to update the ion data. Defer the final calculation of the ion data until the electrons and potential have been advanced.

2. Predict an edge and time centered electron density as well as an implicitly calculated cell centered electron density at the new time. These will be used for the electron flux approximation (3.1).

3. Solve a modified Poisson equation for the new potential. Use the new electric field to update the electron density and flux. 
4. Update the electron internal energy using the new electron data and electric field.

5. Perform a final update on the ion data using a temporally second-order accurate source term.

Our spatial discretization begins with the cell averaging of each of the continuous equations on an underlying uniform grid. The divergence operator then becomes a surface integral operator

$$
\nabla \cdot F \equiv \frac{1}{h_{x} h_{y}} \oint F \cdot d S
$$

The usual differential notation is retained for convenience, though the dependent variables now represent cell averages and the divergence operation has the above interpretation. This conservative finite difference approach allows the evolution of cell averaged quantities to be related to fluxes through cell edges during a timestep. This facilitates the extension of the algorithm to locally refined grids, as will be discussed in later chapters.

We can now describe the single grid algorithm. Given known values for all quantities at time $t_{n}$, we wish to integrate $(2.4)-(2.8)$ up to a new time $t_{n+1}=t_{n}+\Delta t$. The timestep $\Delta t$ is chosen to satisfy the CFL conditions imposed by the explicit steps in the algorithm as described in Section 3.4. We proceed as follows:

\section{Step 1 : Explicit Ion Advection}

The update formula for the ion continuity equation is

$$
\frac{n_{i}^{n+1}-n_{i}^{n}}{\Delta t}=-\nabla \cdot\left(n_{i} u_{i}\right)^{n+1 / 2}+\frac{\nu_{i z}^{n}}{2}\left(n_{e}^{n+1}+n_{e}^{n}\right) .
$$

In the explicit method used to compute the flux divergence $\left(n_{i} u_{i}\right)^{n+1 / 2}$ the lagged field $E^{n}$ and ionization source $\nu_{i z}^{n} n_{e}^{n}$ are used. Since the time-centered ionization source represented by the last term in the right-hand side of (3.3) cannot be computed until $n_{e}^{n+1}$ is known, the final computation of $n_{i}^{n+1}$ is postponed until $n_{e}^{n+1}$ has been computed. We may defer the calculation of $n_{i}^{n+1}$ because the ion density flux term,$\nabla \cdot\left(n_{i} u_{i}\right)^{n+1 / 2}$, is the only part of the ion advance required by the rest of the algorithm. This allows the source term contribution to be temporally second order accurate while eliminating additional couplings to the remaining parts of the time splitting. 
To perform the integration, we notice that the system of ion equations (2.6)-(2.7) can be written as a single vector equation

$$
\frac{\partial U}{\partial t}+\frac{\partial}{\partial x} F(U)+\frac{\partial}{\partial y} G(U)=H\left(U, \phi, n_{e}\right)
$$

where we have the following definitions:

$$
\begin{aligned}
& U=\left[\begin{array}{c}
n_{i} \\
,_{x} \\
,_{y}
\end{array}\right], \quad,{ }_{i}=\left[\begin{array}{l}
{ }_{x} \\
,_{y}
\end{array}\right], \quad,{ }_{x}=n_{i} v_{x}, \quad,{ }_{y}=n_{i} v_{y} \\
& c^{2}=\frac{k T_{i}+k T_{e}}{m_{i}} \\
& F(U)=\left[\begin{array}{c}
,_{x} \\
\frac{x^{2}}{n_{i}}+c^{2} n_{i} \\
\frac{, x, y}{n_{i}}
\end{array}\right] \quad G(U)=\left[\begin{array}{c}
,_{y} \\
\frac{, x y}{n_{i}} \\
\frac{y^{2}}{n_{i}}+c^{2} n_{i}
\end{array}\right] \\
& H\left(U, E, n_{e}\right)=\left[\begin{array}{c}
\nu_{i z} n_{e} \\
\frac{k T_{e}}{m_{i}} \frac{\partial n_{i}}{\partial x}+\frac{e}{m_{i}} n_{i} E_{x}-\nu_{i n},{ }_{x}+\nu_{i z} n_{e},{ }_{x} / n_{i} \\
\frac{k T_{e}}{m_{i}} \frac{\partial n_{i}}{\partial y}+\frac{e}{m_{i}} n_{i} E_{y}-\nu_{i n}, y+\nu_{i z} n_{e},{ }_{y} / n_{i}
\end{array}\right]
\end{aligned}
$$

Note that $v_{x}$ and $v_{y}$ are used in this section to denote velocities while $u$ is reserved for the vector of dependent variables.

We have added a term, $\left(k T_{e} / m_{i}\right) \nabla n_{i}$, to both sides of the momentum equation (2.7). The reason for this is that the characteristic velocity for signal propogation in the medium is the ion acoustic velocity. By adding this term, we may treat the system as in the gas dynamics case. An analogous treatment without this additional term would leave the isothermal sound speed $k T_{i} / m_{i}$ as the characteristic speed in the medium. By adding this term, we may treat the system as an inhomogeneous version of the well understood Euler equations. An analogous treatment using the isothermal sound speed $k T_{i} / m_{i}$ results in incorrect wave speeds and improper upwinding. Adding 
this term gives an additional benefit as well. For ambipolar flow, this term added to the source tends to cancel the other field term (and does so exactly if the ion diffusivity and mobility are negligible compared to that of the electrons). This reduces the magnitude of the source which is treated explicitly, reducing a possible source of stability problems.

We use a second order accurate, unsplit Godunov method [17], which is described below, to solve this system.

\section{The Godunov Procedure}

To cell average (3.4) we apply to each term :

$$
\frac{1}{\Delta t \Delta x \Delta y} \int_{t_{n}}^{t_{n+1}} d t \int_{x_{i-\frac{1}{2}}}^{x_{i+\frac{1}{2}}} d x \int_{y_{j-\frac{1}{2}}}^{y_{j+\frac{1}{2}}} d y
$$

The first term in (3.4) becomes :

$$
\frac{U_{i, j}^{n+1}-U_{i, j}^{n}}{\Delta t}
$$

Where $U_{i, j}^{n}$ is a vector quantity representing the average value in cell $i, j$, at time $t^{n}$, of the pointwise vector quantity $u$.

The second and third terms represent time averaged flux values through cell faces within a timestep. These flux values are obtained by evaluating the flux functions $F(U)$ and $G(U)$ using the solution to local Riemann problems at the cell interfaces (as in [39]). The states used to calculate the Riemann solution are detailed below, but it is important to point out that rather than using cell centered values from the previous time step for these states, we use Taylor series expansions in time and space about the cell centered values for better accuracy. These edge state values are depicted in Figure 3.1 for the case of edges normal to the $x$ direction. Using these states increases the spatial and temporal accuracy to second order (at least in the case of linear advection). If we represent 


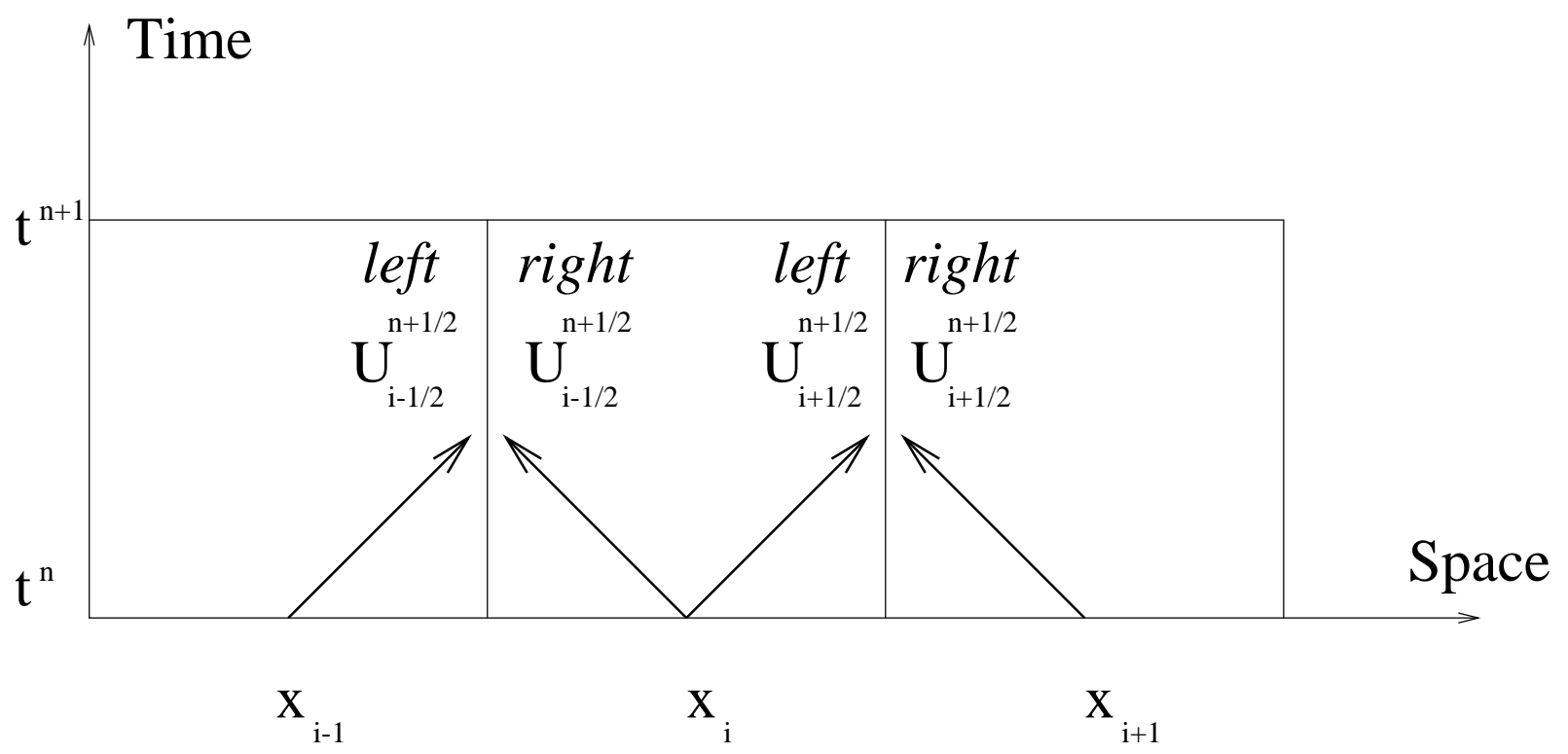

Figure 3.1: Calculation of left and right states for Riemann problems for edges normal to the $x$ direction. Arrows indicate position for state predictions relative to base of Taylor expansion.

these Riemann solutions by $\tilde{u}_{i, j}^{n}$, the second and third terms can be written as :

$$
\begin{aligned}
& \frac{1}{\Delta x}\left[F\left(\tilde{u}_{i+\frac{1}{2}, j}^{n}\right)-F\left(\tilde{u}_{i-\frac{1}{2}, j}^{n}\right)\right] \\
& \frac{1}{\Delta y}\left[G\left(\tilde{u}_{i, j+\frac{1}{2}}^{n}\right)-G\left(\tilde{u}_{i, j-\frac{1}{2}}^{n}\right)\right]
\end{aligned}
$$

The source term $H\left(U, E, n_{e}\right)$ is calculated in two steps. First, the values of the state variables at $t^{n}$ are used to calculate a provisional update at time $t^{n+1}, u_{i, j}^{* n+1}$. The update is recalculated using this value for the source term $H\left(u_{i, j}^{* n+1}, E^{n+1}, n_{e}^{n+1}\right)$. In this way, a more accurate description of the source evolution during the step is obtained. This gives for the fourth term :

$$
\frac{1}{2}\left[H\left(u_{i, j}^{n}, E^{n}, n_{e}^{n}\right)+H\left(u_{i, j}^{* n+1}, E^{n+1}, n_{e}^{n+1}\right)\right]
$$

Rearranging gives the update scheme for the cell averages :

$$
\begin{aligned}
U_{i, j}^{n+1}=U_{i, j}^{n}- & \frac{\Delta t}{\Delta x}\left[F\left(\tilde{u}_{i+\frac{1}{2}, j}^{n}\right)-F\left(\tilde{u}_{i-\frac{1}{2}, j}^{n}\right)\right]-\frac{\Delta t}{\Delta y}\left[G\left(\tilde{u}_{i, j+\frac{1}{2}}^{n}\right)-G\left(\tilde{u}_{i, j-\frac{1}{2}}^{n}\right)\right]+ \\
& +\frac{\Delta t}{2}\left[H\left(u_{i, j}^{n}, E^{n}, n_{e}^{n}\right)+H\left(u_{i, j}^{* n+1}, E^{n+1}, n_{e}^{n+1}\right)\right]
\end{aligned}
$$




\section{The Riemann Solver}

Central to the Godunov update scheme is the solution of Riemann problems at cell boundaries. The solution to the Riemann problem for the ions can be accurately approximated. This is due to the similarity of the equations to the isothermal Euler equations, which have been extensively studied and for which nearly analytic Riemann solutions exist [39].

The Riemann solver returns a solution at a cell edge given the states on both sides. The solver calculates admissible shock speeds and intermediate state solutions from the Rankine-Hugoniot

condition. As in [18] one of the states is chosen if on a shock connection, or a linearly interpolated value is calculated if on a rarefaction connection. The solver is locally one dimensional in that the transverse behavior is included only as a source term in the calculation of the left and right states. The term unsplit is in contrast to methods using Strang splitting [54]. Rather than alternating between directions on subsequent steps, as in a split method, each edge (normal to $x$ or $y$ ) is treated independently and on an equal footing each step.

\section{Half Step Predictions}

In order to calculate the edge fluxes, a Riemann problem must be solved at each cell boundary. If lagged cell centered data is used as input to the Riemann solver, the method is first order (in the case of linear advection). Higher order interpolation in space alone can be used. However this requires twice as many Riemann solves for comparable accuracy [39].

The approach we have chosen is to Taylor expand in space and time about the cell centered value at $t^{n}$ to get a better approximation to the edge states at $t^{n+\frac{1}{2}}$. This allows the Riemann solves to be done, operationally, as in the first order case, but with second order accuracy (this can be shown analytically for the case of linear advection and computationally for Burgers' equation). The difference is that we are using more accurate (spatially and temporally) values for the left and right states in the Riemann solve.

So, the left and right state values for the boundary bordering cells $(i, j)$ and $(i+1, j)$, 
depicted in Figure (3.1), and the bottom and top states for the boundary bordering cells $(i, j)$ and $(i, j+1)$ will be :

$$
\begin{aligned}
& \left.U_{i+\frac{1}{2}, j}^{n+\frac{1}{2}}\right|^{L}=U_{i, j}^{n}+\left.\frac{\Delta t}{2} \frac{\partial U}{\partial t}\right|_{i, j} ^{n}+\left.\frac{\Delta x}{2} \frac{\partial U}{\partial x}\right|_{i, j} ^{n} \\
& U_{i+\frac{1}{2}, j}^{n+\frac{1}{2},\left.\right|^{R}}=U_{i+1, j}^{n}+\left.\frac{\Delta t}{2} \frac{\partial U}{\partial t}\right|_{i+1, j} ^{n}-\left.\frac{\Delta x}{2} \frac{\partial U}{\partial x}\right|_{i+1, j} ^{n} \\
& \left.U_{i, j+\frac{1}{2}}^{n+\frac{1}{2}}\right|^{B}=U_{i, j}^{n}+\left.\frac{\Delta t}{2} \frac{\partial U}{\partial t}\right|_{i, j} ^{n}+\left.\frac{\Delta y}{2} \frac{\partial U}{\partial y}\right|_{i, j} ^{n} \\
& \left.U_{i, j+\frac{1}{2}}^{n+\frac{1}{2}}\right|^{T}=U_{i, j+1}^{n}+\left.\frac{\Delta t}{2} \frac{\partial U}{\partial t}\right|_{i, j+1} ^{n}-\left.\frac{\Delta y}{2} \frac{\partial U}{\partial y}\right|_{i, j+1} ^{n}
\end{aligned}
$$

The time derivatives are then replaced with spatial derivatives using the conservation law (3.4) in quasi-linear form, to obtain

$$
\begin{aligned}
& \left.U_{i+\frac{1}{2}, j}^{n+\frac{1}{2}}\right|^{L}=U_{i, j}^{n}+\left.\frac{\Delta x}{2} \frac{\partial U}{\partial x}\left[I-\frac{\Delta t}{\Delta x} \partial F\right]\right|_{i, j} ^{n}-\left.\frac{\Delta t}{2} G_{y}\right|_{i, j} ^{n}+\left.\frac{\Delta t}{2} H\right|_{i, j} ^{n} \\
& \left.U_{i+\frac{1}{2}, j}^{n+\frac{1}{2}}\right|^{R}=U_{i+1, j}^{n}-\left.\frac{\Delta x}{2} \frac{\partial U}{\partial x}\left[I+\frac{\Delta t}{\Delta x} \partial F\right]\right|_{i+1, j} ^{n}-\left.\frac{\Delta t}{2} G_{y}\right|_{i+1, j} ^{n}+\left.\frac{\Delta t}{2} H\right|_{i+1, j} ^{n} \\
& \left.U_{i, j+\frac{1}{2}}^{n+\frac{1}{2}}\right|^{B}=U_{i, j}^{n}+\left.\frac{\Delta y}{2} \frac{\partial U}{\partial y}\left[I-\frac{\Delta t}{\Delta y} \partial G\right]\right|_{i, j} ^{n}-\left.\frac{\Delta t}{2} F_{x}\right|_{i, j} ^{n}+\left.\frac{\Delta t}{2} H\right|_{i, j} ^{n} \\
& \left.U_{i, j+\frac{1}{2}}^{n+\frac{1}{2}}\right|^{T}=U_{i, j+1}^{n}-\left.\frac{\Delta y}{2} \frac{\partial U}{\partial y}\left[I+\frac{\Delta t}{\Delta y} \partial G\right]\right|_{i, j+1} ^{n}-\left.\frac{\Delta t}{2} F_{x}\right|_{i, j+1} ^{n}+\left.\frac{\Delta t}{2} H\right|_{i, j+1} ^{n}
\end{aligned}
$$

where $\partial F$ and $\partial G$ are the Jacobians of the flux functions $F$ and $G$, respectively, and are given by :

$$
\begin{gathered}
\partial F=\left[\begin{array}{ccc}
0 & 1 & 0 \\
-v_{x}^{2}+c^{2} & 2 v_{x} & 0 \\
-v_{x} v_{y} & v_{y} & v_{x}
\end{array}\right] \\
\partial G=\left[\begin{array}{ccc}
0 & 0 & 1 \\
-v_{x} v_{y} & v_{y} & v_{x} \\
-v_{y}^{2}+c^{2} & 0 & 2 v_{y}
\end{array}\right]
\end{gathered}
$$




\section{Stability}

We wish to further modify these state predictions in order to enhance stability. We first want to use slope limiting to prevent oscillations which could feed into non-linear instabilities. Secondly, we wish to take advantage of local characteristic information and remove portions of the Taylor series expansion which do not come from the "upwind" direction. The first of these objectives is accomplished through the use of the slope limiting operator, $S L$. The upwinding is done with two projection operators $P_{+}$and $P_{-}$. These are described below.

\section{Flux Limiting}

It is well known that some modification must be made to high order fluid methods, near discontinuities or other sharp gradients, in order to prevent catastrophic oscillations [64]. These modifications usually take the form of an artificial viscosity or of slope or flux limiters. Below we describe our slope limiting approach [18] which locally reduces the method to first order in regions where the second order method introduces local extrema.

In the one dimensional case, the VanLeer [58] slope limiter chooses a discretization for spatial derivatives which will not introduce local extrema into the Taylor series predictions. Without loss of generality, we consider the $x$-direction (the $y$-direction follows analogously). For some scalar quantity, $s$, we calculate the limited derivative as follows :

$$
S L_{\text {scalar }}\left(\frac{\partial s}{\partial x}\right)= \begin{cases}\operatorname{sign}\left(\alpha_{c}\right) \min \left(\alpha_{l}, \alpha_{c}, \alpha_{r}\right) & ; \quad \alpha_{l} \alpha_{r}>0 \\ 0 & ; \quad \alpha_{l} \alpha_{r} \leq 0\end{cases}
$$

where

$$
\begin{aligned}
\alpha_{l} & =\left(s_{i}-s_{i-1}\right) / \Delta x \\
\alpha_{c} & =\left(s_{i+1}-s_{i-1}\right) /(2 \Delta x) \\
\alpha_{r} & =\left(s_{i+1}-s_{i}\right) / \Delta x .
\end{aligned}
$$


If the left and right slope calculations differ in sign, this indicates some oscillation is present in the solution. The derivative is therefore set to zero, which drops the state prediction to first order as intended.

In our case, we want to calculate a slope limited derivative of a vector, $\partial U / \partial x$, rather than a scalar. So some generalization must be made in order to use the slope limiter (3.12). This is accomplished by considering an eigenvector expansion for the derivative with slope limiting on the individual coefficients [18]. The eigenvalues and eigenvectors correspond to the Jacobian matrices $\partial F$ in the $x$-direction and $\partial G$ in the $y$-direction. Let $\lambda_{1}, \lambda_{2}$ and $\lambda_{3}$ be the eigenvalues, $r_{1}, r_{2}$ and $r_{3}$ be the right eigenvectors and $l_{1}^{T}, l_{2}^{T}$, and $l_{3}^{T}$ be the left eigenvectors of $\partial F$. Further, require that the eigenvector pairs satisfy the orthonormality condition :

$$
l_{i}^{T} \cdot r_{j}=\delta_{i, j}
$$

The limited derivative is then calculated as follows

$$
S L\left(\frac{\partial U}{\partial x}\right)=\sum_{k=1}^{3} \alpha_{k} r_{k}
$$

where the individual $\alpha^{\prime} s$ in each cell $j$ are given by a new slope limiter generalized as follows

$$
\alpha_{k}= \begin{cases}\operatorname{sign}\left(\alpha_{c}\right) \min \left(\alpha_{l}, \alpha_{c}, \alpha_{r}\right) & ; \alpha_{l} \alpha_{r}>0 \\ 0 & ; \alpha_{l} \alpha_{r} \leq 0\end{cases}
$$

where

$$
\begin{aligned}
\alpha_{k}^{l} & =l_{k}^{T} \cdot\left(U_{j}-U_{j-1}\right) / \Delta x \\
\alpha_{k}^{c} & =l_{k}^{T} \cdot\left(U_{j+1}-U_{j-1}\right) /(2 \Delta x) \\
\alpha_{k}^{r} & =l_{k}^{T} \cdot\left(U_{j+1}-U_{j}\right) / \Delta x
\end{aligned}
$$




\section{Characteristic Projections}

In addition to the limiting described in the previous section, we wish to use "upwinding" principles to improve the state predictions (3.9) as is detailed in [18]. We want to do better than first order Godunov, which just uses the old values for the Riemann inputs, but we do not want to do this at the expense of stability. One way to attain this goal is to only use part of the Taylor series expansion to improve our edge prediction. This is done by forming the correction as an eigenvector expansion and only including the terms which correspond to waves moving in the proper direction. This selection of the upwind component is facilitated with the use of the projection operators $P_{+}$ and $P_{-}$which select the right going waves and left going waves, respectively. They are defined, in each cell, for some vector argument $W$ as

$$
\begin{aligned}
& P_{+}(W)=\sum_{k \mid \lambda_{k}>0}\left(l_{k}^{T} \cdot W\right) r_{k} \\
& P_{-}(W)=\sum_{k \mid \lambda_{k}<0}\left(l_{k}^{T} \cdot W\right) r_{k}
\end{aligned}
$$

Including the slope limiting and characteristic projection operators, we have our final representation of the left/right and top/bottom state values used for the Riemann solution

$$
\begin{aligned}
& \left.U_{i+\frac{1}{2}, j}^{n+\frac{1}{2}}\right|^{L}=U_{i, j}^{n} \quad+\left.\frac{\Delta x}{2} P_{+}\left\{S L\left(\frac{\partial U}{\partial x}\right)\left[I-\frac{\Delta t}{\Delta x} \partial F\right]\right\}\right|_{i, j} ^{n}-\left.\frac{\Delta t}{2} G_{y}\right|_{i, j} ^{n}+\left.\frac{\Delta t}{2} H\right|_{i, j} ^{n} \\
& \left.U_{i+\frac{1}{2}, j}^{n+\frac{1}{2}}\right|^{R}=U_{i+1, j}^{n}-\left.\frac{\Delta x}{2} P_{-}\left\{S L\left(\frac{\partial U}{\partial x}\right)\left[I+\frac{\Delta t}{\Delta x} \partial F\right]\right\}\right|_{i+1, j} ^{n}-\left.\frac{\Delta t}{2} G_{y}\right|_{i+1, j} ^{n}+\left.\frac{\Delta t}{2} H\right|_{i+1, j} ^{n} \\
& \left.U_{i, j+\frac{1}{2}}^{n+\frac{1}{2}}\right|^{B}=U_{i, j}^{n}+\left.\frac{\Delta y}{2} P_{+}\left\{S L\left(\frac{\partial U}{\partial y}\right)\left[I-\frac{\Delta t}{\Delta y} \partial G\right]\right\}\right|_{i, j} ^{n}-\left.\frac{\Delta t}{2} F_{x}\right|_{i, j} ^{n}+\left.\frac{\Delta t}{2} H\right|_{i, j} ^{n} \\
& \left.U_{i, j+\frac{1}{2}}^{n+\frac{1}{2}}\right|^{T}=U_{i, j+1}^{n}-\left.\frac{\Delta y}{2} P_{-}\left\{S L\left(\frac{\partial U}{\partial y}\right)\left[I+\frac{\Delta t}{\Delta y} \partial G\right]\right\}\right|_{i, j+1} ^{n}-\left.\frac{\Delta t}{2} F_{x}\right|_{i, j+1} ^{n}+\left.\frac{\Delta t}{2} H\right|_{i, j+1} ^{n}
\end{aligned}
$$

\section{Two Pass Flux Calculation}

In order to calculate these state predictions, the cell centered transverse flux must be known. For example, the predictions of the left state requires the value of $\left.G_{y}\right|_{i, j} ^{n}$. In order to have 
approximations for these transverse flux terms, a two pass scheme is used.

In the first pass, these transverse flux terms are approximated by ignoring the normal direction and any source terms. So, if we calculate the fluxes associated with the conservation laws :

$$
\begin{aligned}
& \frac{\partial u}{\partial t}+\frac{\partial}{\partial x} F(u)=0 \\
& \frac{\partial u}{\partial t}+\frac{\partial}{\partial y} G(u)=0
\end{aligned}
$$

then we will be able to approximate $\left.G_{y}\right|_{i, j} ^{n}$ and $\left.F_{x}\right|_{i, j} ^{n}$ as :

$$
\begin{aligned}
\left.F_{x}\right|_{i, j} ^{n} & \approx \frac{1}{\Delta x}\left(F\left(\tilde{u}_{i+\frac{1}{2}, j}^{n}\right)-F\left(\tilde{u}_{i-\frac{1}{2}, j}^{n}\right)\right) \\
\left.G_{y}\right|_{i, j} ^{n} & \approx \frac{1}{\Delta y}\left(G\left(\tilde{u}_{i, j+\frac{1}{2}}^{n}\right)-G\left(\tilde{u}_{i, j-\frac{1}{2}}^{n}\right)\right)
\end{aligned}
$$

With these transverse values, we can then calculate the state predictions in (3.24) in a second pass.

\subsubsection{Step 2 : Diffusive Flux Calculation}

To begin the electron advance, we substitute (2.1) into (2.4) to obtain

$$
\frac{\partial n_{e}}{\partial t}=-\nabla \cdot\left(n_{e} \bar{u}_{e}\right)+\nabla \cdot \eta \nabla\left(n_{e} k T_{e}\right)+\nu_{i z} n_{e}
$$

where $\bar{u}_{e} \equiv-\mu E$ represents the lagged electron drift velocity. We then calculate $n_{e}^{n+1 / 2}$, which is a slope-limited, Taylor-series prediction of the edge electron density at $t_{n+1 / 2}$ upwinded relative to the drift velocity $\bar{u}_{e}^{n}$. This upwinding is depicted in Figure 3.2. Only the $x$-direction is shown, the $y$-direction follows analogously. The edge electron densities are then given by

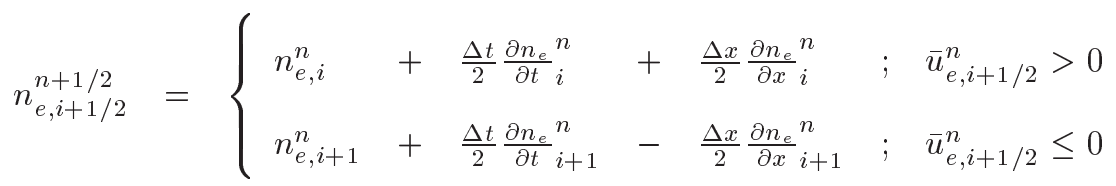




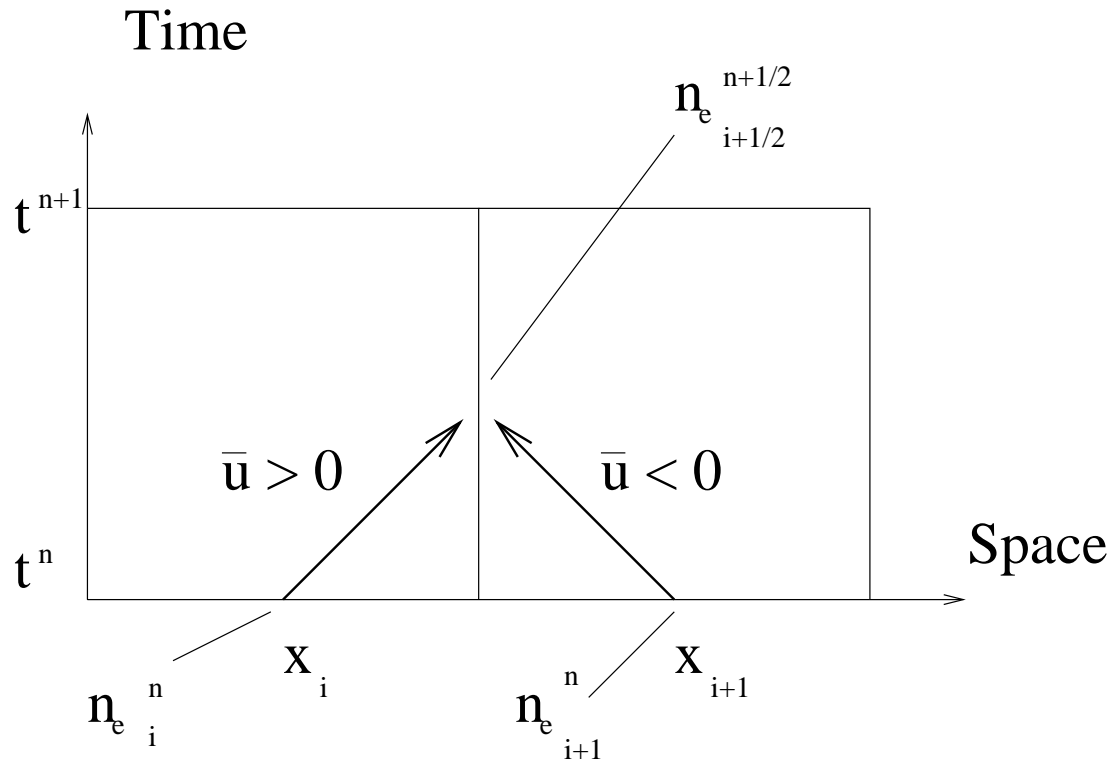

Figure 3.2: Calculation of edge electron densities based on drift velocity direction.

where, as in the ion equations, the temporal derivatives are replaced using the quasi-linear form of the continuity equation and the spatial derivative $\partial n_{e} / \partial x$, which is slope limited.

We then substitute the flux approximation (3.1) into the continuity equation to obtain an equation for $\widehat{n}_{e}^{n+1}$. This is discretized as follows :

$$
\begin{aligned}
& \frac{\widehat{n}_{e}^{n+1}-n_{e}^{n}}{\Delta t}=-\nabla \cdot\left(n_{e}^{n+1 / 2} \bar{u}_{e}^{n}\right) \\
& \quad+\quad \nabla \cdot \eta^{n} \nabla\left(\widehat{n}_{e}^{n+1} k T_{e}^{n}\right)+\frac{\nu_{i z}^{n}}{2}\left(\widehat{n}_{e}^{n+1}+n_{e}^{n}\right)
\end{aligned}
$$

Collecting terms involving $\widehat{n}_{e}^{n+1}$ on the left-hand side yields a linear system that can be solved for this quantity.

\subsubsection{Step 3 : Coupled Potential and Electron Drift Flux Calculation}

Now that we have predictions for the electron density, we can form the modified Poisson equation. Difference the constraint (2.8) to obtain

$$
\frac{\epsilon_{0}}{e} \frac{\nabla^{2} \phi^{n+1}-\nabla^{2} \phi^{n}}{\Delta t}=\frac{n_{e}^{n+1}-n_{e}^{n}}{\Delta t}-\frac{n_{i}^{n+1}-n_{i}^{n}}{\Delta t}
$$


Use the continuity equation to replace the first term on the right-hand side by

$$
\begin{aligned}
& \frac{n_{e}^{n+1}-n_{e}^{n}}{\Delta t}=-\nabla \cdot\left(n_{e}^{n+1 / 2} \bar{u}_{e}^{n+1}\right) \\
& \quad+\nabla \cdot \eta^{n} \nabla\left(\widehat{n}_{e}^{n+1} k T_{e}^{n}\right)+\frac{\nu_{i z}^{n}}{2}\left(n_{e}^{n+1}+n_{e}^{n}\right)
\end{aligned}
$$

where

$$
\bar{u}_{e}^{n+1} \equiv-\mu^{n} E^{n+1}=\mu^{n} \nabla \phi^{n+1} .
$$

Replace the second term on the right-hand side of (3.28) by (3.3). Collecting all terms involving $\phi^{n+1}$ on the left-hand side and multiplying by $\Delta t$, we obtain a modified Poisson equation

$$
\begin{aligned}
\nabla \cdot & {\left[\left(\frac{\epsilon_{0}}{e}+\mu^{n} \Delta t n_{e}^{n+1 / 2}\right) \nabla \phi^{n+1}\right]=} \\
& n_{e}^{n}-n_{i}^{n}+\Delta t \nabla \cdot\left(n_{i} u_{i}\right)^{n+1 / 2}+\nabla \cdot\left(\eta^{n} \Delta t\right) \nabla\left(\widehat{n}_{e}^{n+1} k T_{e}^{n}\right)
\end{aligned}
$$

which is an elliptic problem that can be solved for $\phi^{n+1}$. Using (3.29), the value of $n_{e}^{n+1}$ is then obtained via the formula

$$
\begin{aligned}
n_{e}^{n+1} & =\left(1-\frac{\nu_{i z}^{n} \Delta t}{2}\right)^{-1}\left\{-\nabla \cdot\left(n_{e}^{n+1 / 2} \Delta t \bar{u}_{e}^{n+1}\right)\right. \\
& \left.+\quad \nabla \cdot\left(\eta^{n} \Delta t\right) \nabla\left(\widehat{n}_{e}^{n+1} k T_{e}^{n}\right)+\left(1+\frac{\nu_{i z}^{n} \Delta t}{2}\right) n_{e}^{n}\right\}
\end{aligned}
$$

\subsubsection{Step 4 : Internal Energy Evolution}

We discretize (2.6) as

$$
\begin{gathered}
\frac{3}{2} \frac{n_{e}^{n+1} k T_{e}^{n+1}-n_{e}^{n} k T_{e}^{n}}{\Delta t}+\nabla \cdot\left(\frac{5}{2},{ }_{e}^{n+1} k T_{e}^{n+1 / 2}\right)=-e,{ }_{e}^{n+1} \cdot E^{n+1} \\
+\frac{5}{2} \nabla \cdot\left(\eta^{n+1 / 2}\left(n_{e}^{n+1 / 2} k T_{e}^{n+1 / 2}\right) \nabla\left(k T_{e}^{n+1}\right)\right) \\
+P_{\text {ind }}-\left(\nu_{i z}^{n} \epsilon_{i z}+\nu_{\text {elas }}^{n} \epsilon_{\text {elas }}+\frac{1}{2} m_{e} u_{e}^{n} \cdot u_{e}^{n} \nu_{i z}^{n}\right) n_{e, c c}^{n+1 / 2}
\end{gathered}
$$

Where $n_{e, c c}$ is a cell centered, time centered electron density and $T_{e}^{n+1 / 2}$ is a slope-limited, Taylorseries prediction of the edge temperature at $t_{n+1 / 2}$ upwinded relative to the velocity $u_{e}^{n}$ (similarly 
to 3.26$)$ where

$$
\begin{aligned}
E^{n+1 / 2} & =-\nabla\left(\phi^{n+1}+\phi^{n}\right) / 2 \\
{ }_{e}^{n+1} & =-n_{e}^{n+1 / 2} \mu^{n} E^{n+1}-\eta^{n} \nabla\left(n_{e}^{n+1} k T_{e}\right)
\end{aligned}
$$

Collecting all terms involving $T_{e}^{n+1}$ on the left-hand side, we obtain a linear system that can be solved for this quantity. Multiplication by $n_{e}^{n+1}$ and Boltzmann's constant then gives the new internal energy.

\subsubsection{Step 5 : Final Update for Ions}

At this point, all that remains is the final update of the ion data. To do this, we must evaluate the source term $H\left(U, E, n_{e}\right)$ in equation (3.4). At the start of the timestep we have ion data

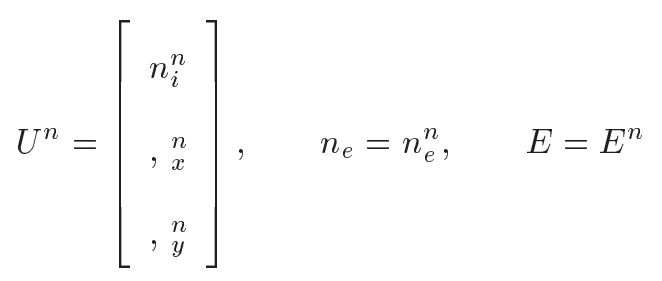

At the end of Step 3, we have provisional values for the ion data and final values for the electron density and electric field

$$
\widehat{U}^{n+1}=\left[\begin{array}{c}
\widehat{n}_{i}^{n+1} \\
\widehat{,}_{x}^{n+1} \\
\widehat{,}_{y}^{n+1}
\end{array}\right], \quad n_{e}=n_{e}^{n+1}, \quad E=E^{n+1}
$$

We then average the source term evaluated with these two states. This is much like Heun's method [21] and is second order accurate in time. With this source term we can calculate the new solution to the conservation law $(3.4)$

$$
\begin{gathered}
U^{n+1}=U^{n}-\frac{\Delta t}{\Delta x}\left[F\left(\tilde{U}_{R}^{n+1 / 2}\right)-F\left(\tilde{U}_{L}^{n+1 / 2}\right)\right]-\frac{\Delta t}{\Delta y}\left[G\left(\tilde{U}_{T}^{n+1 / 2}\right)-G\left(\tilde{U}_{B}^{n+1 / 2}\right)\right]+ \\
+\frac{\Delta t}{2}\left[H\left(U^{n}, E^{n}, n_{e}^{n}\right)+H\left(\widehat{U}^{n+1}, E^{n+1}, n_{e}^{n+1}\right)\right]
\end{gathered}
$$


where $\tilde{U}_{R}^{n+1 / 2}, \tilde{U}_{L}^{n+1 / 2}, \tilde{U}_{T}^{n+1 / 2}$ and $\tilde{U}_{B}^{n+1 / 2}$ are Riemann solutions for the evaluation of time centered fluxes on the right, left, top and bottom cell edges, respectively.

\subsection{Boundary Conditions}

For the potential $\phi$, Dirichlet boundary conditions are imposed corresponding to an applied voltage at the reactor walls.

For the electron number density $n_{e}$ and temperature $T_{e}$, boundary conditions are required for the electron flux $n_{e} u_{e}$. Consider a spatial cell with an edge on the problem boundary. Let ${ }_{b}$ denote the outward normal component of $n_{e} u_{e}$ at the center of the boundary edge, and let $\phi_{b}$ denote the applied voltage there. Let $\phi, n_{e}$ and $T_{e}$ denote the potential, electron number density and electron temperature at the cell center, respectively. Of all the electrons at the cell center, only those moving toward the boundary with kinetic energies greater than $e\left|\phi_{b}-\phi\right|$ can overcome the potential barrier. Using a Maxwellian distribution

$$
f(w)=\left(\frac{m_{e}}{2 \pi k T_{e}}\right)^{\frac{1}{2}} \exp \left(\frac{-m_{e} w^{2}}{2 k T_{e}}\right)
$$

we therefore propose that

$$
{ }_{b}{ }_{b}=n_{e} \bar{w}_{e}\left(k T_{e}, \phi\right)
$$

where $\bar{w}_{e}$ is the average velocity of electrons reaching the boundary and is given by :

$$
\begin{aligned}
\bar{w}_{e}\left(k T_{e}, \phi\right) & \equiv \int_{\left(\frac{2 e\left|\phi_{b}-\phi\right|}{m_{e}}\right)^{\frac{1}{2}}}^{\infty} w f(w) d w \\
& =\sqrt{\frac{k T_{e}}{2 \pi m_{e}}} \exp \left(-\frac{e\left|\phi_{b}-\phi\right|}{k T_{e}}\right) \\
& =\frac{\bar{v}\left(k T_{e}\right)}{4} \exp \left(-\frac{e\left|\phi_{b}-\phi\right|}{k T_{e}}\right)
\end{aligned}
$$

where $\bar{v}\left(k T_{e}\right)=\sqrt{8 k T_{e} / \pi m_{e}}$ is the average speed for a Maxwellian distribution. Let $n_{e}^{n+1 / 2}$ again denote the Taylor-series-predicted density at the boundary. We can then require that at the problem boundary

$$
n_{e}^{n+1 / 2} \bar{u}_{e}^{k}-\eta^{n} \nabla\left(n_{e}^{k} k T_{e}^{n}\right)=n_{e}^{k} \bar{w}_{e}\left(k T_{e}^{n}, \phi^{k}\right) \quad k=n, n+1
$$


which provides boundary conditions for the corresponding terms in (3.27), (3.32) and (3.33). It should be noted that this relation for the total flux does not give the drift and diffusive components separately, but only their sum. This presents a difficulty in that the total flux appears in both the diffusive flux calculation 3.27 and the modified potential equation 3.32. If we want to keep these solutions decoupled, we cannot implicitely calculate the drift and diffusive flux at the boundary simultaneously. We choose to use the lagged value for the total electron flux at the boundary in solving 3.27. The potential dependent total flux 3.42 is then calculated self-consistently in the solution to 3.32 .

The solution of the linear system represented by (3.33) requires a boundary condition for the total energy flux, $Q_{b}$. The total energy is calculated by arguments similar to those in (3.41). A derivation for the total energy flux is shown in [40] and is given by

$$
Q_{b}=\frac{5}{2} n_{e} k T_{e} u_{e}-\frac{5 \eta}{2} n_{e} k T_{e} \nabla k T_{e}=2 k T_{e}, b
$$

For the ions, we use Godunov's method which requires the solution of Riemann problems at all cell edges during a timestep. These Riemann solutions give the nonlinear equivalent of upwind solutions which are used to evaluate the flux functions $F$ and $G$ in (3.4). On interior edges, predictions are obtained on both sides of each edge for these Riemann problems. Instead of solving such a problem at physical boundaries, we instead assume that the prediction coming from the interior of the domain is the solution to the Riemann problem.

This has an interesting physical interpretation. Provided the ions are flowing outward at a velocity greater than the ion acoustic velocity, $\sqrt{k T_{i} / m_{i}+k T_{e} / m_{i}}$, then the choice of the interior prediction as the Riemann solution is correct. Since the ion velocity at the wall exceeds the Bohm velocity, $\sqrt{k T_{e} / m_{i}}$, and $T_{i} \ll T_{e}$ this should be an accurate boundary condition. 


\subsection{Timestep Restrictions}

The coupling of the electron drift flux with the potential in the solution of (3.32) allows us to exceed the very restrictive dielectric relaxation timestep limit. The dielectric relaxation time $\tau_{\text {dielectric }}$ is given by

$$
\tau_{\text {dielectric }}=\nu_{e n} / \omega_{p}^{2}
$$

where $\nu_{e n}$ is the electron neutral collision frequency and $\omega_{p}$ is the plasma frequency. For our parameters, this can be as small as $10^{-15} \mathrm{~s}$. The explicit nature of the electron density in our time splitting is only conditionally stable, however. We still have a timestep limitation. It was our expectation that we would be limited by the Courant condition based on the electron drift velocity

$$
\Delta t \leq \min \left(\Delta x / v_{x}^{d r i f t}, \Delta y / v_{y}^{d r i f t}\right)
$$

where

$$
\begin{aligned}
& v_{x}^{d r i f t}=-\mu E_{x} \\
& v_{y}^{d r i f t}=-\mu E_{y}
\end{aligned}
$$

Both experience with our code and a linearized stability analysis have confirmed this expectation. The stability analysis for our algorithm (and some variations), as well as a derivation of the dielectric relaxation time, is described in detail in Appendix A. 


\section{Chapter 4}

\section{Extension of the Algorithm to}

\section{Locally Refined Grids}

We have now constructed a time dependent algorithm for a single Cartesian grid. In this chapter we discuss the extension of the algorithm to a locally refined grid of the type shown in Figures 1.2 and 1.1. We begin with some nomenclature describing the grid structure. Important concepts are introduced by first looking at Poisson's equation on two levels. The solution method for the full plasma system is then described, also for two levels. Emphasis is placed on two synchronization steps, one for the coupled electron/potential equations and one for the electron internal energy. We then generalize this description to an arbitrary number of levels and discuss the details of the solution method for the composite synchronizations.

\subsection{Nomenclature}

Let $\Omega_{c}$ denote a union of disjoint, uniform, rectangular grids. Let $\Omega_{f}$ also denote a union of disjoint, uniform, rectangular grids, each of which is obtained by refining a rectangular subgrid of $\Omega_{c}$ by a factor $n_{r e f}$. Let $P\left(\Omega_{f}\right)$ denote the projection of the fine grid $\Omega_{f}$ onto the coarse grid $\Omega_{c}$. 

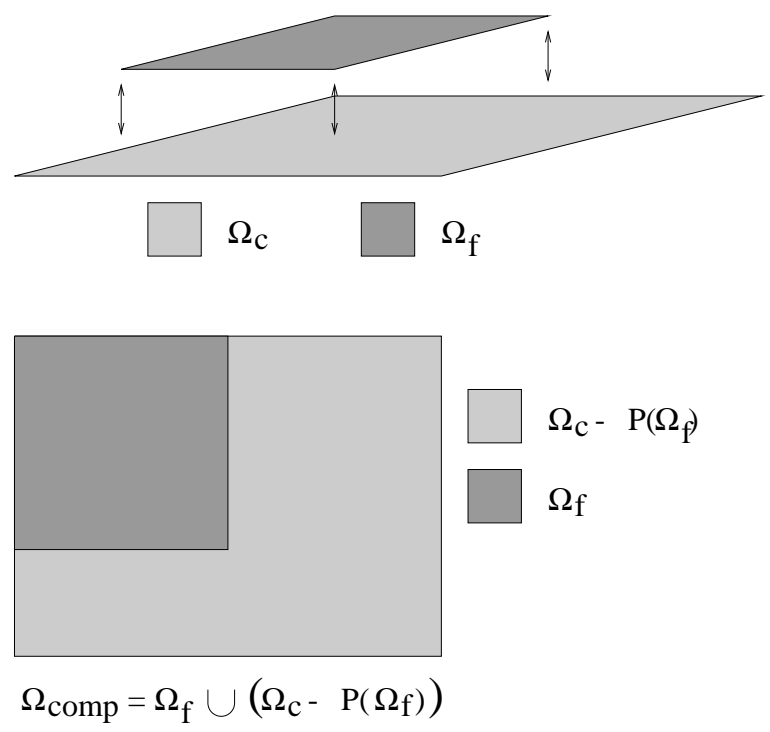

Figure 4.1: Domain definitions for the composite grid. The top figure shows a "side" view of the fine and coarse grids separately, while the bottom figure shows a view from "above" of the composite grid. The composite grid is the union of the fine grid and that portion of the coarse grid not covered by the fine grid.

We define this projection as the region of the coarse grid sharing the same physical space as the fine grid but containing larger cells. Define the composite grid by

$$
\Omega_{c o m p} \equiv \Omega_{f} \cup\left(\Omega_{c}-P\left(\Omega_{f}\right)\right)
$$

and let $I$ denote the interface between the coarse grid component $\Omega_{c}-P\left(\Omega_{f}\right)$ and the fine grid component $\Omega_{f}$ of $\Omega_{c o m p}$. These domains are represented in Figure 4.1. The union of rectangular grids sharing the same cell dimensions is termed a level. Then $\Omega_{f}$ is a level as is $\Omega_{c}$. We refer to $\Omega_{c}$ as the base level and $\Omega_{f}$ as a refined level.

\subsection{Solution of Poisson's Equation on a Composite Grid}

We now want to extend the algorithm described in the preceding section for use on the composite grid (4.1). A key requirement is the enforcement of Poisson's equation

$$
-\epsilon_{0} \Delta \phi=\rho
$$


at each time step, so we begin by describing what it means to solve (4.2) on (4.1). As in the single-grid case, we will not be solving (4.2) directly during the integration of the fluid system on the composite grid, except for initialization or diagnostic purposes. Nevertheless, the separate consideration of (4.2) here will introduce some important concepts and notation.

On $\Omega_{f}$ or $\Omega_{c}$, the standard 5-point cell-centered discretization of (4.2) can be expressed in terms of edge-centered fields as

$$
\epsilon_{0}\left(-E_{i-1 / 2, j}+E_{i+1 / 2, j}-E_{i, j-1 / 2}+E_{i, j+1 / 2}\right)=h \rho_{i, j}
$$

where, e.g.,

$$
E_{i+1 / 2, j} \equiv-\left(\phi_{i+1, j}-\phi_{i, j}\right) / h,
$$

$\phi_{i, j}$ and $\rho_{i, j}$ are the potential and charge at cell center $(i, j)$, respectively, and $h$ is the grid size. Dirichlet conditions are imposed on the physical boundary by extrapolating interior potential values and the prescribed boundary value to a "ghost" cell exterior to the problem, as is depicted in Figure $4.2(\mathrm{a})$.

A composite discretization of (4.2) on (4.1) is obtained by applying (4.3) separately on $\Omega_{f}$ and $\Omega_{c}-P\left(\Omega_{f}\right)$ with the definition of the edge-centered fields on $I$ suitably modified to enforce continuity of the potential and field across the interface. Since each coarse grid edge on $I$ is the union of $n_{r e f}$ fine grid edges, field continuity requires that the coarse fields at such edges be obtained as the average of the fields on the corresponding fine edges.

To obtain the fine grid fields on $I$, we require potentials on $\Omega_{c}-P\left(\Omega_{f}\right)$ interpolated to the fine grid resolution and centered in a single layer of ghost cells surrounding $\Omega_{f}$, which then enables (4.4) to also be applied on fine grid edges contained in $I$. We employ an interpolation scheme whose stencil is depicted in Figure 4.2(b) for a sample grid structure with $n_{r e f}=4$. The potentials at coarse cell centers (large filled circles) are first quadratically interpolated tangentially to $I$ to obtain values at the locations depicted by the unfilled circles. These values are then linearly interpolated normally to $I$ using the potential values at the centers of the fine cells abutting $I$ (small filled circles) 
to obtain potentials at the ghost cell centers depicted by the triangles and squares. As suggested by the overlapping triangle and rectangle in the upper right-hand corner of the coarse grid region in Figure 4.2(b), the potentials interpolated in this way may not be unique, with the value denoted by the triangle being used to compute the field on the vertical fine edge to the right, and the value denoted by the square being used to compute the field on the horizonal fine edge immediately above.

The resulting discretization of (4.2) on $\Omega_{\text {comp }}$ is second-order accurate. The corresponding discrete Laplacian operator is not symmetric, however, as can be deduced from Figure 4.2(c), which displays the operator stencil based at the circled fine grid cell abutting $I$. For example, the coarse cell labeled "a" participates in the stencil based at the circled fine grid cell, but the converse is not the case. Effective multilevel algorithms can nevertheless be employed to solve the discrete system, as will be described in Section 4.3 .4 below. These multilevel algorithms rely heavily upon the observation that the solution $\phi$ of the composite Poisson equation is also obtainable as the solution of a coupled pair of Poisson equations on $\Omega_{f}$ and $\Omega_{c}$

$$
\begin{aligned}
& -\epsilon_{0} \Delta \phi_{f}=\rho_{f} \equiv \rho \quad \text { on } \Omega_{f}, \quad \phi_{f}=Q\left(\phi_{f}, \phi_{c}\right) \text { at } I \\
& -\epsilon_{0} \Delta \phi_{c}= \begin{cases}\rho-\epsilon_{0} \nabla \cdot \delta E & \text { on } \Omega_{c}-P\left(\Omega_{f}\right) \\
\left\langle\rho_{f}\right\rangle_{P\left(\Omega_{f}\right)} & \text { on } P\left(\Omega_{f}\right)\end{cases}
\end{aligned}
$$

by setting

$$
\phi= \begin{cases}\phi_{c} & \text { on } \Omega_{c}-P\left(\Omega_{f}\right) \\ \phi_{f} & \text { on } \Omega_{f}\end{cases}
$$

Here, $Q$ denotes the linear boundary operator at $I$ implicitly defined by the previously described high-order tangential interpolation of coarse grid potentials $\phi_{c}$ combined with normal interpolation of fine grid potentials $\phi_{f}$. $\left\langle\rho_{f}\right\rangle_{P\left(\Omega_{f}\right)}$ denotes the volume-weighted average of $\rho$ onto $P\left(\Omega_{f}\right)$. In (4.6),

$$
\delta E \equiv-\left\langle\nabla \phi_{f}\right\rangle_{I}+\nabla \phi_{c}
$$

where $\langle\cdot\rangle_{I}$ denotes the arithmetic averaging of fine edge data to coarse edge data on $I$. Here, and in the remainder of this section, we adopt the convention that $\delta F$ denotes the difference on coarse 


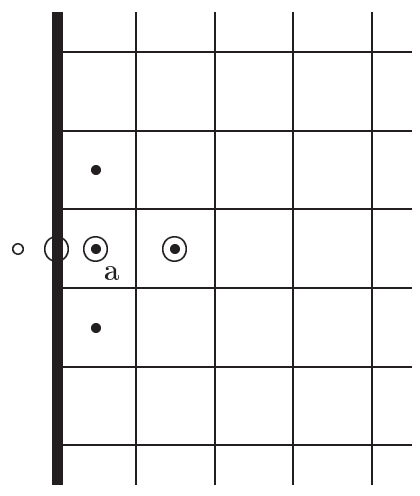

(a)

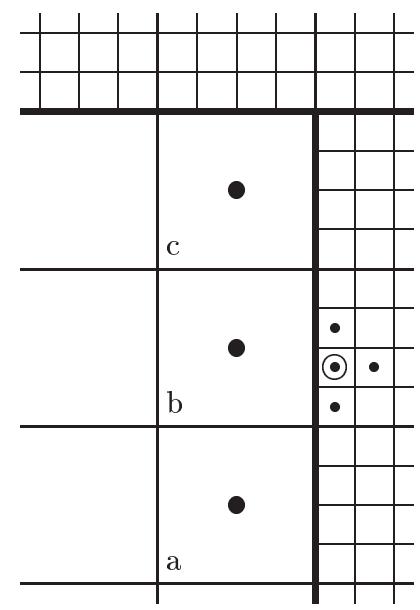

(c)

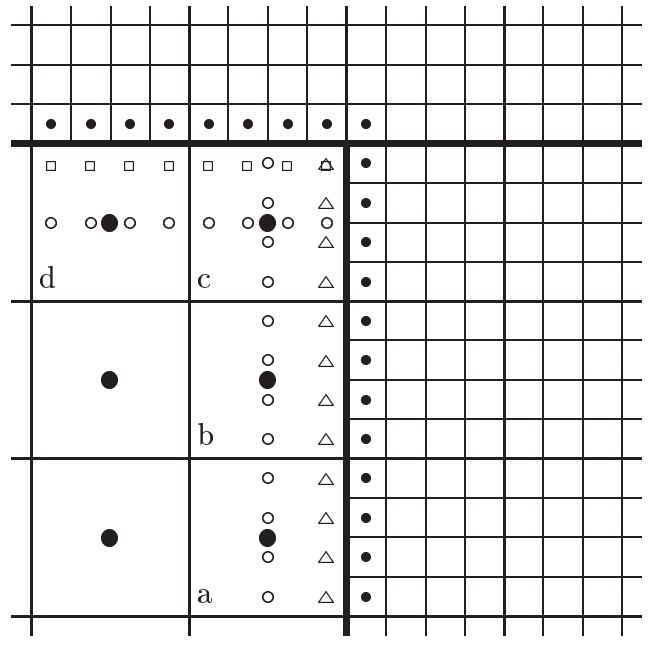

(b)

Figure 4.2: (a) At a physical boundary, interior and boundary values (large o's) are used to extrapolate to the ghost cell (०); the ghost value and the other interior values ( $\bullet$ 's) are used to construct the Laplacian at (a). (b) Locations of coarse grid boundary conditions (large $\bullet$ ), tangentially interpolated values (०), fine grid cells $(\bullet)$, and ghost cells (small $\triangle$ 's and small $\square$ 's). (c) Domain of dependence ( $\bullet$ 's and large $\bullet$ 's) of the Laplacian at a fine cell (large $\circ$ ) adjacent to the coarse/fine interface. 
edges in $I$ of a generic quantity $F$ computed on the coarse grid and fine grids. For such quantities, it is also convenient to define the divergence $\nabla \cdot \delta F$ as the divergence of the extension by zero of $\delta F$ to the remaining edges of $\Omega_{c}$. The term $\epsilon_{0} \nabla \cdot \delta E$ can be regarded as an integrated surface charge, but in fact results solely from the change of grid resolution at $I$. In the solution of (4.6), this "coarse-fine" surface charge causes a jump in the coarse grid field at $I$ such that continuity of the composite field is obtained.

Because only the coarse grid potential $\phi_{c}$ on $\Omega_{c}-P\left(\Omega_{f}\right)$ is used in constructing the composite solution (4.7), it might at first seem inefficient to be solving (4.6) on the entire coarse grid $\Omega_{c}$ versus solving just on $\Omega_{c}-P\left(\Omega_{f}\right)$ and $\Omega_{f}$ with matching conditions at $I$. This is not the case, however, since we intend to solve (4.5)-(4.6) with a multilevel method in which the approximate solutions of (4.6) in residual-correction form computed on $P\left(\Omega_{f}\right)$ represent coarse grid corrections to smoothed approximate solutions of (4.5). This results in a very effective iterative solution method. This approach also avoids the need to compute solutions only on $\Omega_{c}-P\left(\Omega_{f}\right)$, which may be far more geometrically complex than the either of the grids $\Omega_{c}$ or $\Omega_{f}$.

\subsection{Solution of the Plasma Fluid System on a Composite Grid}

\subsubsection{Synchronization of the Charge and Potential}

With the composite solution of Poisson's equation as background, let us now consider the integration of the plasma fluid system. As in the single grid case, discrete time evolution equations on the composite grid can be obtained by combining a first-order difference of Poisson's equation

over a timestep $\Delta t=t^{n+1}-t^{n}$ with the continuity equations for the ions and electrons. Again treating the electron drift flux implicitly, this yields

$$
n_{e}^{n+1}=n_{e}^{n}-\Delta t \nabla \cdot\left(, d_{i f t}\left(n_{e}^{n+1 / 2}, \phi^{n+1}\right)+, d_{i f f}\right)+\Delta t S^{n}
$$




$$
\begin{gathered}
n_{i}^{n+1}=n_{i}^{n}-\Delta t \nabla \cdot,{ }_{i}+\Delta t S^{n} \\
\nabla \cdot\left(\frac{\epsilon_{0}}{e} \nabla \phi^{n+1}+\Delta t,{ }_{\text {drift }}\left(n_{e}^{n+1 / 2}, \phi^{n+1}\right)\right)=n_{e}^{n}-n_{i}^{n}+\Delta t\left(\nabla \cdot,{ }_{i}-\nabla \cdot, \text { diff }^{n}\right)
\end{gathered}
$$

in addition to evolution equations for the ion momentum and electron temperature, which we omit for now. In (4.9) and (4.10), $S^{n}$ denotes the ionization source.

Implied in (4.9)-(4.11) are matching conditions at $I$ which couple the dependent variables on $\Omega_{f}$ and $\Omega_{c}-P\left(\Omega_{f}\right)$. Conservation of mass (i.e., hyperbolic matching conditions) requires that

$$
,_{i, c}=\left\langle{ }_{i, f}\right\rangle_{I},,_{e, c}=\langle, e, f\rangle_{I} .
$$

We further require that the individual components of electron flux (drift and diffusive) are conserved

$$
{ }_{\text {drift }, c}=\left\langle{ }_{\text {drift }, f}\right\rangle_{I},{ }_{\text {diff }, c}=\left\langle, \text { diff }, f_{I} .\right.
$$

Continuity of the potential and field (i.e., second-order elliptic operator matching conditions) is achieved by enforcing

$$
\begin{aligned}
\phi_{f}^{n+1} & =Q\left(\phi_{f}^{n+1}, \phi_{c}^{n+1}\right) \\
n \cdot \nabla \phi_{c}^{n+1} & =\left\langle n \cdot \nabla \phi_{f}^{n+1}\right\rangle_{I}
\end{aligned}
$$

on $I$, where $n$ is a unit normal to $I$.

Although the composite system could be integrated directly using (4.9)-(4.11) and (4.12)(4.15), such an approach would be penalized by the need to use a single timestep for both spatial grid resolutions. In particular, given comparable electron drift velocities, the timestep will be limited by the stability requirements on $\Omega_{f}$, which might represent only a very small fraction of the physical domain. To avoid the resulting unnecessary additional work on $\Omega_{c}-P\left(\Omega_{f}\right)$, we instead employ a predictor-corrector approach comprised of separate integrations on the coarse and fine grids coupled by a composite sychronization step. Specifically, we use the single grid algorithm of Chapter 3 to 
integrate the plasma fluid system on $\Omega_{c}$ over a timestep $\Delta t_{c}=t^{n+1}-t^{n}$. We then integrate the system on $\Omega_{f}$ over the same time interval $t^{n}$ to $t^{n+1}$ using $n_{\text {cycle }}$ uniform time steps $\Delta t_{f} \equiv \Delta t_{c} / n_{\text {cycle }}$, with boundary values interpolated spatially and temporally from $\Omega_{c}-P\left(\Omega_{f}\right)$. We then solve for a potential and electron density correction on the composite grid to restore matching conditions at the coarse-fine interface $I$ that were violated during the separate coarse and fine integrations. The corrected coarse and fine predictions are then combined to obtain a composite solution that closely approximates, with mild assumptions, the solution one would have obtained by integrating the composite system using the fine grid timesteps everywhere.

\section{Desired Composite Solution}

Letting $m=m(n), \ldots, m(n+1)$ index the time steps taken on the fine grid, the solution we would obtain if we solved only the composite problem is given by

$$
\begin{gathered}
n_{e}^{m(n+1)}=n_{e}^{m(n)}-\sum_{m} \Delta t_{f}\left[\nabla \cdot\left(,{ }_{\text {drift }}\left(n_{e}^{m+1 / 2}, \phi^{m+1}\right)+,{ }_{\text {diff }}^{m+1 / 2}\right)-S^{m}\right] \\
n_{i}^{m(n+1)}=n_{i}^{m(n)}-\sum_{m} \Delta t_{f}\left(\nabla \cdot{ }_{i}^{m+1 / 2}-S^{m}\right) \\
\nabla \cdot\left(\frac{\epsilon_{0}}{e} \nabla \phi^{m(n+1)}+\sum_{m} \Delta t_{f}, d_{\text {drift }}\left(n_{e}^{m+1 / 2}, \phi^{m+1}\right)\right) \\
=n_{e}^{m(n)}-n_{i}^{m(n)}+\sum_{m} \Delta t_{f}\left(\nabla \cdot,_{i}^{m+1 / 2}-\nabla \cdot, \begin{array}{l}
m+1 / 2 \\
\text { diff }
\end{array}\right)
\end{gathered}
$$

with the conditions analogous to (4.12)-(4.15) on $I$.

\section{Calculated Coarse Level Solution}

We now consider the system we actually advance and how it differs from 4.16-4.18.

On $\Omega_{c}$, select a timestep $\Delta t_{c}=t^{n+1}-t^{n}$ and perform the integration

$$
\begin{aligned}
& n_{e, c}^{n+1}=n_{e, c}^{n}-\Delta t_{c} \nabla \cdot\left(,{ }_{\text {drift }}\left(n_{e, c}^{n+1 / 2}, \phi_{c}^{n+1}\right)+, \text { diff }, c\right)+\Delta t_{c} S_{c}^{n} \\
& n_{i, c}^{n+1}=n_{i, c}^{n}-\Delta t_{c} \nabla \cdot,{ }_{i, c}+\Delta t_{c} S_{c}^{n}
\end{aligned}
$$




$$
\begin{aligned}
\nabla \cdot\left(\frac{\epsilon_{0}}{e} \nabla \phi_{c}^{n+1}\right. & \left.+\Delta t_{c}, \text { drift }\left(n_{e, c}^{n+1 / 2}, \phi_{c}^{n+1}\right)\right)=\frac{\epsilon_{0}}{e} \nabla \cdot \delta E^{n} \\
& \quad+\Delta t_{c} \nabla \cdot\left(n_{e, c}^{n+1 / 2} \mu^{n} \delta E^{n}\right)+n_{e}^{n}-n_{i}^{n}+\Delta t_{c}\left(\nabla \cdot,_{i, c}-\nabla \cdot, \text { diff }, c\right)
\end{aligned}
$$

This is the same single-level integration described in Chapter 3 except for the first two terms in the right-hand side of (4.21) involving $\delta E^{n}$, which is computed as in (4.8) using $\phi_{f}^{n}$ and $\phi_{c}^{n}$. We include the $\delta E^{n}$ terms in (4.21) as approximations of the analogous quantities at the new time $t^{n+1}$ to anticipate better the effect of the subsequent fine grid integrations. As will be seen later, this modification of the coarse grid prediction reduces the magnitude of the correction required in the composite synchronization step.

\section{Calculated Fine Level Solution}

Next, we integrate the system on $\Omega_{f}$ over the same time interval $t^{n}$ to $t^{n+1}$ using $n_{\text {cycle }}$ uniform time steps $\Delta t_{f} \equiv \Delta t_{c} / n_{\text {cycle }}$. Let $m=m(n), \ldots, m(n+1)$ index the time steps taken on the fine grid. We have

$$
\begin{gathered}
n_{e, f}^{m(n+1)}=n_{e, f}^{m(n)}-\sum_{m} \Delta t_{f}\left[\nabla \cdot\left(,{ }_{\text {drift }}\left(n_{e, f}^{m+1 / 2}, \phi_{f}^{m+1}\right)+, \begin{array}{l}
m+1 / 2 \\
\text { diff }, f
\end{array}\right)-S_{f}^{m}\right] \\
n_{i, f}^{m(n+1)}=n_{i, f}^{m(n)}-\sum_{m} \Delta t_{f}\left(\nabla \cdot,{ }_{i, f}^{m+1 / 2}-S_{f}^{m}\right) \\
\nabla \cdot\left(\frac{\epsilon_{0}}{e} \nabla \phi_{f}^{m(n+1)}+\sum_{m} \Delta t_{f}, d_{\text {drift }}\left(n_{e, f}^{m+1 / 2}, \phi_{f}^{m+1}\right)\right) \\
=n_{e}^{m(n)}-n_{i}^{m(n)}+\sum_{m} \Delta t_{f}\left(\nabla \cdot,_{i, f}^{m+1 / 2}-\nabla \cdot \begin{array}{l}
m+1 / 2 \\
\text { diff } f, f
\end{array}\right)
\end{gathered}
$$

In performing this integration, boundary values are needed at the coarse-fine interface $I$. These values are obtained from the corresponding values on $\Omega_{c}-P\left(\Omega_{f}\right)$ by linear interpolation in time and appropriate spatial interpolations. Specifically, potential boundary values at $I$ are given by

$$
\phi_{f}^{m}=Q\left(\phi_{f}^{m}, \frac{m(n+1)-m}{n_{\text {cycle }}} \phi_{c}^{n}+\frac{m-m(n)}{n_{\text {cycle }}} \phi_{c}^{n+1}\right), \quad m=m(n)+1, \ldots, m(n+1)
$$

Boundary values for the fine grid electron and ion data at $I$ are obtained from conservative spatial interpolation of their corresponding values on $\Omega_{c}-P\left(\Omega_{f}\right)$, linearly interpolated in time, to a layer of ghost cells surrounding $\Omega_{f}$. 


\section{Approximating the Composite Solution from the Calculated Coarse and Fine Solutions}

Having integrated the fluid system on both $\Omega_{f}$ and $\Omega_{c}-P\left(\Omega_{f}\right)$, we would like to compose a solution of (4.16)-(4.18) from the integrated solutions obtained from (4.19)-(4.21) and (4.22)-(4.24). However, it is not possible to do this immediately for two reasons. First, the interpolated Dirichlet boundary condition (4.25) only ensures that the potential is continuous at $I$, but nothing has been done to enforce field continuity there. In other words, Poisson's equation is not yet satisfied since the composite potential still needs to be adjusted to satisfy also the interface conditions (4.15). Secondly, since different fluxes were used on $I$ during the integration of the coarse and fine grids, (4.12) does not necessarily hold and conservation must be restored by an appropriate "refluxing" of ions and electrons. Such a redistribution of charge again implies a modification of the composite potential.

One solution to this dilema would be to iterate the coarse and fine grid integrations until we obtain a composite solution which satisfies the hyperbolic and elliptic matching conditions. This is clearly computationally unattractive. Another alternative would be to determine corrections to the fine and coarse solutions in order to obtain a solution satisfying the matching conditions. If we were to formally subtract the coarse and fine solutions from the composite solution, we would hope to obtain an equation for these corrections. With very mild approximations, this is what we indeed obtain. The approximations we make are based on three principles.

The first principle is that the inertia of the ions makes them insensitive to changes in the potential over the relatively small timesteps, which are determined by the significantly higher electron mobility. The ion density and momenta will not be immediately affected by small changes in the field. Consequently, we consider charge redistribution due to composite potential corrections as entirely due to a change in the electron density. It is also sufficient to include field corrections in the momentum source term (3.5) in subsequent timesteps.

Secondly, for quasi-neutral plasmas, perturbations to the electron density (for a single coarse step) are very important as they effect the space charge, but are not important for quantities 
involving the density alone. For example, a change of one part in one thousand for the electron density will have little effect on the calculated pressure gradient, but may drastically change the space charge. This observation is used in three approximations. First, the edge centered electron densities used in the fine and coarse modified Poisson solves are approximately equal to those we would have if we were integrating the composite system itself. The same can be said regarding the diffusive flux terms used for the fine and coarse integrations. Thirdly, the change in ion and electron density due to ionization does not affect the net charge and therefore does not require correction after the separate integration of the coarse and fine grid systems.

Our final observation is that, as described in Chapter 3, the electron temperature and the dependent rate coefficients $\nu_{e n}$ and $\nu_{i z}$ change slowly over a timestep. We may therefore neglect changes in the rate coefficients due to the composite corrections.

With these assumptions, we proceed in deriving equations for the potential and electron density corrections which allow us to satisfy the governing equations along with the hyperbolic and elliptic matching conditions.

We seek a potential correction $\phi^{*}=\left(\phi_{f}^{*}, \phi_{c}^{*}\right)$ satisfying the composite system obtained by substituting

$$
\phi^{m} \equiv\left\{\begin{array}{ll}
\phi_{f}^{m}+\phi_{f}^{*} & \text { on } \Omega_{f} \\
\phi_{c}^{n+1}+\phi_{c}^{*} & \text { on } \Omega_{c}-P\left(\Omega_{f}\right)
\end{array} \quad m=m(n)+1, \ldots, m(n+1)\right.
$$

into (4.18), then subtracting (4.21) on $\Omega_{c}-P\left(\Omega_{f}\right)$ and (4.24) on $\Omega_{f}$. The correction must also satisfy the continuity requirement

$$
\phi_{f}^{*}=Q\left(\phi_{f}^{*}, \phi_{c}^{*}\right)
$$

at the coarse-fine boundary $I$. Let us now determine the equation to be satisfied by $\phi^{*}$ on $\Omega_{c}-P\left(\Omega_{f}\right)$. On edges of $\Omega_{c}-P\left(\Omega_{f}\right)$ contained in $I$, define

$$
\begin{aligned}
\delta,_{i} & \equiv\left\langle\frac{1}{\Delta t_{c}} \sum_{m} \Delta t_{f}, \begin{array}{l}
m+1 / 2 \\
i, f
\end{array}\right\rangle_{I}-,_{i, c} \\
\delta,,_{\text {diff }} & \equiv\left\langle\frac{1}{\Delta t_{c}} \sum_{m} \Delta t_{f}, \begin{array}{l}
m+1 / 2 \\
\text { diff }, f
\end{array}\right\rangle_{I}-, \text { diff } f, c
\end{aligned}
$$


Substituting (4.26) into (4.18), with $\Delta t=\Delta t_{c}$, then subtracting (4.21) on $\Omega_{c}-P\left(\Omega_{f}\right)$, we obtain that

$$
\begin{aligned}
\nabla & \cdot\left[\frac{\epsilon_{0}}{e} \nabla\left(\phi^{m(n+1)}-\phi_{c}^{n+1}\right)+\Delta t,{ }_{\text {drift }}^{*}\right] \\
& =-\frac{\epsilon_{0}}{e} \nabla \cdot \delta E^{n}-\Delta t \nabla \cdot\left(n_{e, c}^{n+1 / 2} \mu^{n} \delta E^{n}\right)+\Delta t \nabla \cdot\left(\delta,{ }_{i}-\delta, \text { diff }\right)
\end{aligned}
$$

where

$$
{ }_{d r i f t}^{*} \equiv\left\{\begin{array}{c}
\left\langle\frac{1}{\Delta t_{c}} \sum_{m} \Delta t_{f}, d_{\text {rift }}\left(n_{e}^{m+1 / 2}, \phi^{m+1}\right)\right\rangle_{I}-,{ }_{d r i f t}\left(n_{e, c}^{n+1 / 2}, \phi_{c}^{n+1}\right) \\
\text { on edges of } \Omega_{c}-P\left(\Omega_{f}\right) \text { contained in } I \\
\frac{1}{\Delta t_{c}} \sum_{m} \Delta t_{f}, d r i f t\left(n_{e}^{m+1 / 2}, \phi^{m+1}\right)-, d r i f t\left(n_{e, c}^{n+1 / 2}, \phi_{c}^{n+1}\right) \\
\text { on edges of } \Omega_{c}-P\left(\Omega_{f}\right) \text { not contained in } I
\end{array}\right.
$$

In the first term of (4.30), we observe that

$$
\nabla\left(\phi^{m(n+1)}-\phi_{c}^{n+1}\right)= \begin{cases}\nabla \phi_{c}^{*} & \text { on edges of } \Omega_{c}-P\left(\Omega_{f}\right) \text { not contained in } I \\ \left\langle\nabla \phi_{f}^{*}\right\rangle_{I} & \text { on edges of } \Omega_{c}-P\left(\Omega_{f}\right) \text { contained in } I\end{cases}
$$

On edges of $\Omega_{c}-P\left(\Omega_{f}\right)$ not contained in $I$ and not part of the physical boundary, (4.31) yields

$$
,_{d r i f t}^{*}=n_{e, c}^{n+1 / 2} \mu^{n} \nabla \cdot \phi_{c}^{*}
$$

Using (4.31), on the edges of $\Omega_{c}-P\left(\Omega_{f}\right)$ contained in $I$, we have

$$
\begin{aligned}
,_{\text {drift }}^{*}= & \left\langle\frac{1}{\Delta t_{c}} \sum_{m} \Delta t_{f} n_{e}^{m+1 / 2} \mu^{m}\left(\nabla \phi_{f}^{m+1}+\nabla \phi^{*}\right)\right\rangle_{I}-n_{e, c}^{n+1 / 2} \mu^{n} \nabla \phi_{c}^{n+1} \\
\approx & \left\langle\frac{1}{\Delta t_{c}} \sum_{m} \Delta t_{f}, d_{\text {rift }}\left(n_{e, f}^{m+1 / 2}, \phi_{f}^{m+1}\right)\right\rangle_{I}-, \text { drift }\left(n_{e, c}^{n+1 / 2}, \phi_{c}^{n+1}\right) \\
& +\left\langle n_{e}^{m(n+1)-1 / 2} \mu^{m(n+1)-1}\right\rangle_{I}\left\langle\nabla \phi^{*}\right\rangle_{I}
\end{aligned}
$$

in which we make the approximation

$$
\left\langle n_{e}^{m+1 / 2} \mu^{m} \nabla \phi^{*}\right\rangle_{I} \approx\left\langle n_{e}^{m+1 / 2} \mu^{m}\right\rangle_{I}\left\langle\nabla \phi^{*}\right\rangle_{I}
$$


Therefore, letting

$$
\operatorname{drift}\left(\phi_{c}^{*}\right) \equiv \begin{cases}n_{e}^{n+1 / 2} \mu^{n} \nabla \phi_{c}^{*} & \text { on edges of } \Omega_{c}-P\left(\Omega_{f}\right) \text { not contained in } \\ \left\langle n_{e, f}^{m(n+1)-1 / 2} \mu^{m(n+1)-1}\right\rangle_{I} \nabla \phi_{c}^{*} & \text { on edges of } \Omega_{c}-P\left(\Omega_{f}\right) \text { contained in } I\end{cases}
$$

on the edges of $\Omega_{c}-P\left(\Omega_{f}\right)$ not contained in the physical boundary, and defining

$$
\begin{aligned}
\delta E^{n+1} \equiv & -\left\langle\nabla \phi_{f}^{n+1}\right\rangle_{I}+\nabla \phi_{c}^{n+1} \\
\delta,_{e} \equiv & \left\langle\frac{1}{\Delta t_{c}} \sum_{m} \Delta t_{f}, \text { drift }\left(n_{e, f}^{m+1 / 2}, \phi_{f}^{m+1}\right)\right\rangle_{I}-,_{\text {drift }}\left(n_{e, c}^{n+1 / 2}, \phi_{c}^{n+1}\right) \\
& +n_{e, c}^{n+1 / 2} \mu^{n} \delta E^{n}+\delta, \text { diff }
\end{aligned}
$$

on the edges of $\Omega_{c}-P\left(\Omega_{f}\right)$ contained in $I,(4.30)$ becomes

$$
\begin{aligned}
& \nabla \cdot\left(\frac{\epsilon_{0}}{e} \nabla \phi_{c}^{*}+\Delta t,\right.\text { drift } \left.\left(\phi_{c}^{*}\right)\right)= \\
&- \nabla \cdot\left(\left(\frac{\epsilon_{0}}{e}+\left\langle\Delta t n_{e, f}^{m(n+1)-1 / 2} \mu^{m(n+1)-1}\right\rangle_{I}\right)\left(\left\langle\nabla \phi_{f}^{*}\right\rangle_{I}-\nabla \phi_{c}^{*}\right)\right) \\
&+ \frac{\epsilon_{0}}{e} \nabla \cdot\left(\delta E^{n+1}-\delta E^{n}\right)+\Delta t \nabla \cdot\left(\delta,{ }_{i}-\delta,{ }_{e}\right) \\
& \phi_{c}^{*}=0 \quad \text { on the physical boundary }
\end{aligned}
$$

To obtain the equation determining $\phi^{*}$ on $\Omega_{f}$, we subtract (4.24) from (4.18), which yields

$$
\nabla \cdot\left(\frac{\epsilon_{0}}{e} \nabla \phi_{f}^{*}+\Delta t,{ }_{d r i f t}^{*}\right)=0, \quad \phi_{f}^{*}= \begin{cases}Q\left(\phi_{f}^{*}, \phi_{c}^{*}\right) & \text { at } I, \\ 0 & \text { on the physical boundary }\end{cases}
$$

where

$$
{ }_{d r i f t}^{*} \equiv \frac{1}{\Delta t_{c}} \sum_{m} \Delta t_{f}\left[{ }_{d r i f t}\left(n_{e}^{m+1 / 2}, \phi^{m+1}\right)-,{ }_{d r i f t}\left(n_{e, f}^{m+1 / 2}, \phi_{f}^{m+1}\right)\right]
$$

On the non-boundary edges of $\Omega_{f},{ }_{d r i f t}^{*}$ is approximated by

$$
,{ }_{d r i f t}\left(\phi_{f}^{*}\right) \equiv n_{e, f}^{m(n+1)-1 / 2} \mu^{m(n+1)-1} \nabla \phi_{f}^{*}
$$


Hence, on $\Omega_{f}$,

$$
\nabla \cdot\left(\frac{\epsilon_{0}}{e} \nabla \phi_{f}^{*}+\Delta t, \text { drift }_{f}\left(\phi_{f}^{*}\right)\right)=0, \quad \phi_{f}^{*}=\left\{\begin{array}{ll}
Q\left(\phi_{f}^{*}, \phi_{c}^{*}\right) & \text { at } I, \\
0 & \text { on the physical boundary }
\end{array} .\right.
$$

The pair of equations (4.40) and (4.44) thus form the system to be solved for the composite potential correction $\phi^{*}$. It only remains to specify the modifications to be made on physical boundary edges due to (3.40). On the edges of $\Omega_{c}-P\left(\Omega_{f}\right)$ on the physical boundary, (4.31) implies that

$$
\begin{aligned}
& n \cdot{ }_{d \text { drift }}^{*} \\
& \qquad=\frac{1}{\Delta t_{c}} \sum_{m} \Delta t_{f} n_{e, c}^{m+1 / 2} \frac{\bar{v}_{e}^{m}}{4} \exp \left[-\frac{e\left(\phi^{m+1}-\phi_{b}^{m+1}\right)}{k T_{e}^{m}}\right]-n_{e, c}^{n+1 / 2} \frac{\bar{v}_{e}^{n}}{4} \exp \left[-\frac{e\left(\phi_{c}^{n+1}-\phi_{b}^{m+1}\right)}{k T_{e}^{n}}\right] \\
& \quad \approx n_{e, c}^{n+1 / 2} \frac{\bar{v}_{e}^{n}}{4}\left\{\frac{1}{\Delta t_{c}} \sum_{m} \Delta t_{f} \exp \left[-\frac{e\left(\phi^{m+1}-\phi_{b}^{m+1}\right)}{k T_{e}^{n}}\right]-\exp \left[-\frac{e\left(\phi_{c}^{n+1}-\phi_{b}^{m+1}\right)}{k T_{e}^{n}}\right]\right\} \\
& \quad=n_{e, c}^{n+1 / 2} \frac{\bar{v}_{e}^{n}}{4} \exp \left[-\frac{e\left(\phi_{c}^{n+1}-\phi_{b}^{m+1}\right)}{k T_{e}^{n}}\right]\left[\exp \left(-\frac{e \phi^{*}}{k T_{e}^{n}}\right)-1\right] \\
& \quad=n \cdot,{ }_{\text {drift }}\left(n_{e, c}^{n+1 / 2}, \phi_{c}^{n+1}\right)\left[\exp \left(-\frac{e \phi^{*}}{k T_{e}^{n}}\right)-1\right]
\end{aligned}
$$

and similarly on the boundary edges in $\Omega_{f}$. Thus, (4.37) and (4.43) are supplemented by

$$
\begin{aligned}
n \cdot, \operatorname{drift}_{t}\left(\phi_{c}^{*}\right) & \equiv n \cdot, \operatorname{drift}_{t}\left(n_{e, c}^{n+1 / 2}, \phi_{c}^{n+1}\right)\left[\exp \left(-\frac{e}{k T_{e}^{n}} \phi_{c}^{*}\right)-1\right] \\
n \cdot, \operatorname{drift}\left(\phi_{f}^{*}\right) & \equiv n \cdot, \operatorname{drift}\left(n_{e, f}^{m(n+1)-1 / 2}, \phi_{f}^{m(n+1)}\right)\left[\exp \left(-\frac{e}{k T_{e}^{n}} \phi^{*}\right)-1\right]
\end{aligned}
$$

respectively.

Similar subtractions of the ion and electron continuity equations yield that

$$
\begin{aligned}
& n_{i}^{m(n+1)} \equiv \begin{cases}n_{i, f}^{m(n+1)} & \text { on } \Omega_{f} \\
n_{i, c}^{n+1}-\Delta t \nabla \cdot \delta, i & \text { on } \Omega_{c}-P\left(\Omega_{f}\right)\end{cases} \\
& n_{e}^{m(n+1)} \equiv \begin{cases}n_{e, f}^{m(n+1)}-\Delta t \nabla \cdot,{ }_{\text {drift }}\left(\phi^{*}\right) & \text { on } \Omega_{f} \\
n_{e, c}^{n+1}-\Delta t \nabla \cdot\left(,{ }_{\text {drift }}\left(\phi^{*}\right)+\delta,{ }_{e}\right) & \text { on } \Omega_{c}-P\left(\Omega_{f}\right)\end{cases}
\end{aligned}
$$

satisfy (4.16)-(4.17).

In the single level integration algorithm, the electron diffusive flux, diff is computed using a prediction $\widehat{n}_{e}^{n+1}$ of the electron density obtained by solving (3.27). In solving this linear system, 
it is convenient to regard $\widehat{n}_{e}^{n+1} k T_{e}^{n}$ as the dependent variable. We therefore require a boundary value for this quantity for the corresponding linear solves occuring during the fine grid integration (4.22)-(4.24). For this, we again employ linear interpolation in time and the high-order spatial interpolation operator $Q$, i.e.,

$$
\widehat{n}_{e, f}^{m+1} k T_{e, f}^{m}=Q\left(\widehat{n}_{e, f}^{m+1} k T_{e, f}^{m}, \frac{m(n+1)-m}{n_{c y c l e}} n_{e, c}^{n} k T_{e, c}^{n+1}+\frac{m-m(n)}{n_{c y c l e}} n_{e, c}^{n+1} k T_{e, c}^{n+1}\right)
$$

for $m=m(n)+1, \ldots, m(n+1)$. Note that, analogous to the single level integration, we do not correct the diffusive flux following the composite correction step (4.49).

Restoration of ion momentum conservation is handled similarly to (4.48). Specifically, momentum flux differences are accumulated on $I$ during the coarse and fine level integrations, then used to correct the coarse level ion momenta. This correction is independent of all other composite synchronization steps.

\subsubsection{Synchonization of the Electron Internal Energy}

The composite synchronization of the coarse and fine level integrated electron temperatures is performed in a manner similar to the potential correction.

\section{Desired Composite Solution}

The composite solution we desire is given by

$$
\begin{aligned}
\frac{3}{2} n_{e}^{m(n+1)} k T_{e}^{m(n+1)}-\frac{3}{2} n_{e}^{m(n)} k T_{e}^{m(n)}+\sum_{m} \Delta t_{f} \nabla \cdot\left(\frac{5}{2},{ }_{e}^{m+1 / 2} k T_{e}^{m+1 / 2}\right) \\
=\sum_{m} \Delta t_{f}\left[\nabla \cdot\left(\frac{5 \eta^{m}}{2} n_{e}^{m+1 / 2} k T_{e}^{m+1 / 2} \nabla k T_{e}^{m+1}\right)+P_{n e t}^{m}\right]
\end{aligned}
$$

where

$$
P_{\text {net }}^{m} \equiv-e,{ }^{m} \cdot E^{m}+P_{\text {ind }}^{m}-\left(\nu_{i z}^{m} \epsilon_{i z}+\nu_{\text {elas }}^{m} \epsilon_{\text {elas }}+\frac{1}{2} m_{e} u_{e}^{m} \cdot u_{e}^{m} \nu_{i z}^{m}\right) n_{e}^{m}
$$




\section{Calculated Coarse Level Solution}

After the integration (4.19)-(4.21) of the potential and electron density over $\Delta t=\Delta t_{c}$ on $\Omega_{c}$, the temperature equation is also integrated to obtain

$$
\begin{aligned}
\frac{3}{2} n_{e, c}^{n+1} k T_{e, c}^{n+1}-\frac{3}{2} n_{e}^{n} k T_{e}^{n} & +\Delta t \nabla \cdot\left(\frac{5}{2},{ }_{e, c}^{n+1 / 2} k T_{e, c}^{n+1 / 2}\right) \\
=\Delta t & {\left[\nabla \cdot\left(\frac{5 \eta_{c}^{n}}{2} n_{e, c}^{n+1 / 2} k T_{e, c}^{n+1 / 2} \nabla k T_{e, c}^{n+1}\right)+P_{n e t}^{n}\right] }
\end{aligned}
$$

where

$$
P_{\text {net }}^{n} \equiv-e,{ }_{e}^{n} \cdot E^{n}+P_{\text {ind }}^{n}-\left(\nu_{i z}^{n} \epsilon_{i z}+\nu_{\text {elas }}^{n} \epsilon_{\text {elas }}+\frac{1}{2} m_{e} u_{e}^{n} \cdot u_{e}^{n} \nu_{i z}^{n}\right) n_{e}^{n}
$$

\section{Calculated Fine Level Solution}

After the integration (4.22)-(4.24) of the potential and electron density on $\Omega_{f}$, the temperature equation is integrated to obtain

$$
\begin{aligned}
\frac{3}{2} n_{e, f}^{m(n+1)} k T_{e, f}^{m(n+1)}-\frac{3}{2} n_{e}^{m(n)} k T_{e}^{m(n)}+\sum_{m} \Delta t_{f} \nabla \cdot\left(\frac{5}{2},{ }_{e, f}^{m+1 / 2} k T_{e, f}^{m+1 / 2}\right) \\
=\sum_{m} \Delta t_{f}\left[\nabla \cdot\left(\frac{5 \eta_{f}^{m}}{2} n_{e}^{m+1 / 2} k T_{e, f}^{m+1 / 2} \nabla k T_{e, f}^{m+1}\right)+P_{n e t}^{m}\right]
\end{aligned}
$$

where

$$
P_{\text {net }}^{m} \equiv-e,{ }_{e, f}^{m} \cdot E_{f}^{m}+P_{i n d}^{m}-\left(\nu_{i z}^{m} \epsilon_{i z}+\nu_{\text {elas }}^{m} \epsilon_{\text {elas }}+\frac{1}{2} m_{e} u_{e}^{m} \cdot u_{e}^{m} \nu_{i z}^{m}\right) n_{e, f}^{m}
$$

with boundary values at $I$ interpolated from the fine grid for $m=m(n)+1, \ldots, m(n+1)$ :

$$
T_{e, f}^{m}=Q\left(T_{e, f}^{m}, \frac{m(n+1)-m}{n_{\text {cycle }}} T_{e, c}^{n}+\frac{m-m(n)}{n_{\text {cycle }}} T_{e, c}^{n+1}\right)
$$

\section{Approximating the Composite Solution from the Calculated Coarse and Fine Solutions}

The potential and electron density are then corrected by solving (4.40) and (4.44) and performing the updates (4.26) and (4.49). Let $n_{e}^{*}$ and,${ }_{e}^{*}$ denote the corrections made to the electron density and flux, respectively, during this step $\left(e . g ., n_{e, c}^{*}=n_{e}^{n+1}-n_{e, c}^{n+1}\right)$. We then seek a composite 
temperature correction $T_{e}^{*}=\left(T_{e, f}^{*}, T_{e, c}^{*}\right)$ such that

$$
T_{e}^{m} \equiv\left\{\begin{array}{ll}
T_{e, f}^{m}+T_{e, f}^{*} & \text { on } \Omega_{f} \\
T_{e, c}^{n+1}+T_{e, c}^{*} & \text { on } \Omega_{c}-P\left(\Omega_{f}\right)
\end{array} \quad m=m(n)+1, \ldots, m(n+1)\right.
$$

with

$$
\begin{aligned}
T_{e, f}^{*} & =Q\left(T_{e, f}^{*}, T_{e, c}^{*}\right) \text { at } I \\
\nabla T_{e}^{*} & =0 \text { at the physical boundary }
\end{aligned}
$$

approximates the solution of the composite system integrated over the same fine grid time steps.

Using the fact that

$$
\begin{aligned}
n_{e}^{n+1} T_{e}^{n+1}-n_{e, c}^{n+1} T_{e, c}^{n+1} & =n_{e}^{n+1} T_{e}^{*}+n_{e}^{n+1} T_{e, c}^{n+1}-n_{e, c}^{n+1} T_{e, c}^{n+1} \\
& =n_{e}^{n+1} T_{e}^{*}+n_{e}^{*} T_{e, c}^{n+1},
\end{aligned}
$$

and making approximations similar to those used in computing the potential correction, subtraction of (4.53) from (4.51) on $\Omega_{c}-P\left(\Omega_{f}\right)$ yields

$$
\begin{aligned}
\frac{3}{2} n_{e}^{n+1} k T_{e, c}^{*}-\Delta t \nabla \cdot\left(\frac{5 \eta^{n}}{2} n_{e}^{n+1 / 2} k T_{e}^{n+1 / 2} \nabla k T_{e, c}^{*}\right)=-\frac{3}{2} n_{e}^{*} k T_{e, c}^{n+1} \\
+\Delta t \nabla \cdot\left[\left\langle\frac{5 \eta^{n}}{2} n_{e}^{n+1 / 2} k T_{e}^{n+1 / 2}\right\rangle_{I}\left(\left\langle\nabla k T_{e, f}^{*}\right\rangle_{I}-\nabla k T_{e, c}^{*}\right)-\frac{5}{2},{ }_{e}^{*} k T_{e}^{n+1 / 2}+\delta Q\right]
\end{aligned}
$$

where

$$
\begin{aligned}
\delta Q & =\left\langle\frac{1}{\Delta t_{c}} \sum_{m} \Delta t_{f}\left[\nabla \cdot\left(\frac{5}{2},{ }_{e, f}^{m+1 / 2} k T_{e, f}^{m+1 / 2}\right)-\frac{5 \eta_{f}^{m}}{2} n_{e, f}^{m+1 / 2} k T_{e, f}^{m+1 / 2} \nabla k T_{e, f}^{m+1}\right]\right\rangle_{I} \\
& -\nabla \cdot\left(\frac{5}{2},{ }_{e, c}^{n+1 / 2} k T_{e, c}^{n+1 / 2}\right)+\frac{5 \eta_{c}^{n}}{2} n_{e, c}^{n+1 / 2} k T_{e, c}^{n+1 / 2} \nabla k T_{e, c}^{n+1},
\end{aligned}
$$

Furthermore, the subtraction of (4.55) from (4.51) on $\Omega_{f}$ yields

$$
\begin{aligned}
\frac{3}{2} n_{e}^{m(n+1)} k T_{e, f}^{*}-\Delta t \nabla & \cdot\left(\frac{5 \eta^{m(n+1)-1}}{2} n_{e}^{m(n+1)-1 / 2} k T_{e}^{m(n+1)-1 / 2} \nabla k T_{e, f}^{*}\right)= \\
& -\frac{3}{2} n_{e}^{*} k T_{e, f}^{m(n+1)}-\Delta t \nabla \cdot\left(\frac{5}{2},{ }_{e}^{*} k T_{e}^{m(n+1)-1 / 2}\right)
\end{aligned}
$$

The pair (4.61) and (4.63) together with (4.59) is therefore the composite system to be solved for the correction $T_{e}^{*}$. 


\subsubsection{Generalization to an Arbitrary Number of Refinement Levels}

The algorithm just described for two grid levels can be generalized to an arbitrary number of levels. Let $\left\{\Omega_{\ell}, \ell=0, \ldots, \ell_{\max }\right\}$ denote a refinement hierarchy with successive levels related as in the two-level case by

$$
P\left(\Omega_{\ell}\right) \subset \Omega_{\ell-1}, \quad \ell=1, \ldots, \ell_{\max }
$$

where $P$ is a projection operator. Each $\Omega_{\ell}$ is again the union of disjoint, rectangular grids obtained by refining rectangular subgrids of $\Omega_{\ell-1}$ by a factor $n_{r e f, \ell}$. For this general case, we also require the proper nesting of levels. Specifically, we stipulate that $P\left(\Omega_{\ell}\right)$ be properly contained in $\Omega_{\ell-1}$ for $\ell=1, \ldots, \ell_{\max }$, except perhaps at the physical domain boundary.

Our two-level algorithm consisted of the integration of the coarse grid equations over a timestep, followed by $n_{\text {cycle }}$ fine grid integrations over the same coarse timestep and a composite synchronization of the two levels, For the general case, this can be implemented recursively, with the composite potential and temperature corrections occurring at the end of timesteps at each level (except the finest level) and involving all finer levels. Figure 4.3 schematically depicts the timestep advance and composite synchronization schedule for a 4-level problem with $n_{r e f, 0}=n_{r e f, 1}=2$ and $n_{r e f, 2}=4$. The horizontal arrows denote single-level time advance steps, while the vertical arrows denote synchronization of the composite grid.

A perhaps subtle detail in our description of the two-level algorithm was the use of approximations such as

$$
\frac{1}{\Delta t_{c}} \sum_{m} \Delta t_{f} n_{e}^{m+1 / 2} \mu^{m} \approx n_{e}^{m(n+1)-1 / 2} \mu^{m(n+1)-1}
$$

which replace the average of fine edge data over the coarse timestep with the data at the last fine timestep. If such approximations are not made, then in the case of more than two grid levels the composite synchronizations would require the similar accumulation and averaging of fine edge data on a given level $\ell$ over all coarser timesteps $\Delta t_{\ell^{\prime}}, \ell^{\prime}=0, \ldots, \ell-1$, since level $\ell$ will eventually participate in a composite synchronization with each of these coarser levels. Although it is possible 


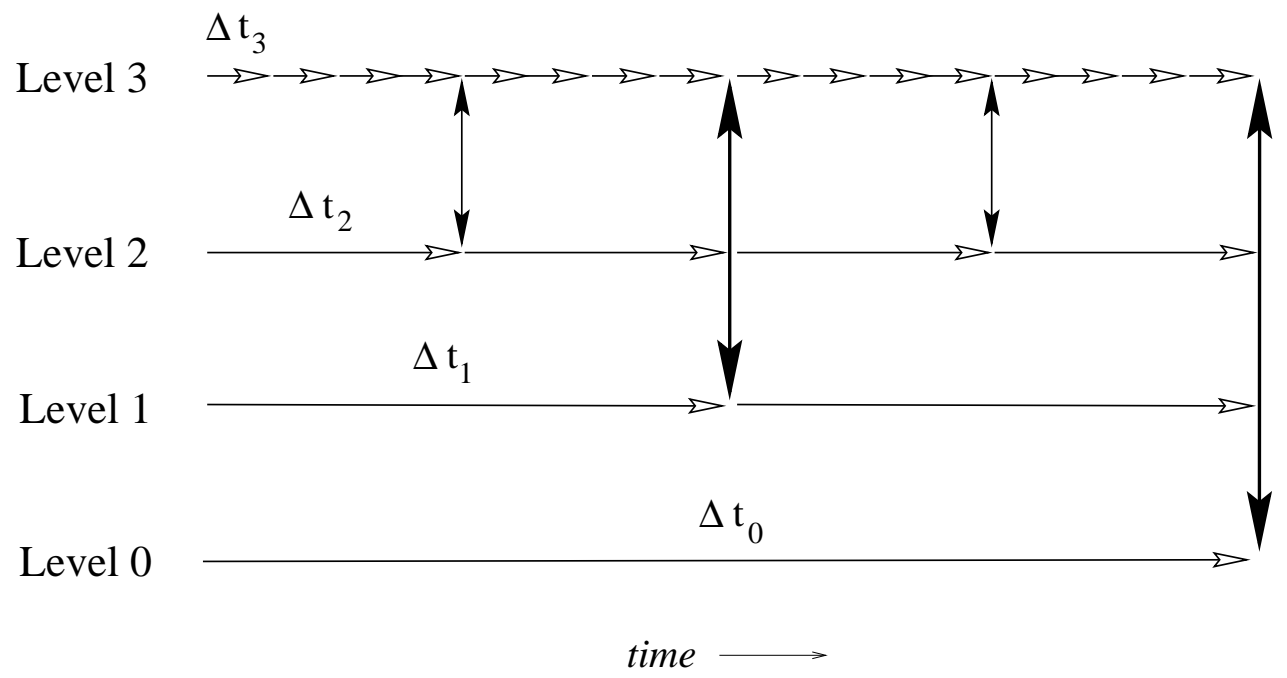

Figure 4.3: Multilevel advance and composite synchronization schedule.

to do this, we have determined empirically that approximations such as (4.65), which enable a totally recursive implementation, do not harm the accuracy of the method.

\subsubsection{Solution of the Composite Systems}

The synchronization of potential and electron temperature lead to the composite systems (4.40)-(4.44) and (4.61)-(4.63), respectively. In this section, we discuss the solution of these systems.

The system (4.40)-(4.44) is nonlinear due to the boundary condition (3.40) on the electron flux. We therefore employ a composite Newton iteration. In each Newton iteration, we must solve a composite Jacobian system with the composite nonlinear residual as the right-hand side, where on each refinement level $\Omega_{\ell}$ the Jacobian has the form of a second-order, symmetric, elliptic operator

$$
L_{\ell}^{n f}\left(\phi_{\ell}\right) \equiv-\nabla \cdot\left(b_{\ell} \nabla \phi_{\ell}\right)+a_{\ell} \phi_{\ell}
$$

Since the level operators of the composite temperature system (4.61)-(4.63) are also of the form (4.66), it suffices to consider the solution of composite systems with operators $L$ of this general form on each level. We generalize the approach described in [43] for Poisson's equation to these variable coefficient cases. This is a multilevel algorithm that iterates over a grid hierarchy $\left\{\Omega_{\ell}, \ell=\right.$ 
$\left.\ell_{\text {base }}, \ldots, \ell_{\max }\right\}$ in a multigrid-like fashion, taking care to enforce the required matching conditions at the boundaries between successive levels. Letting $f$ denote the composite system right-hand side and $u$ denote the desired composite solution, the algorithm can be summarized as

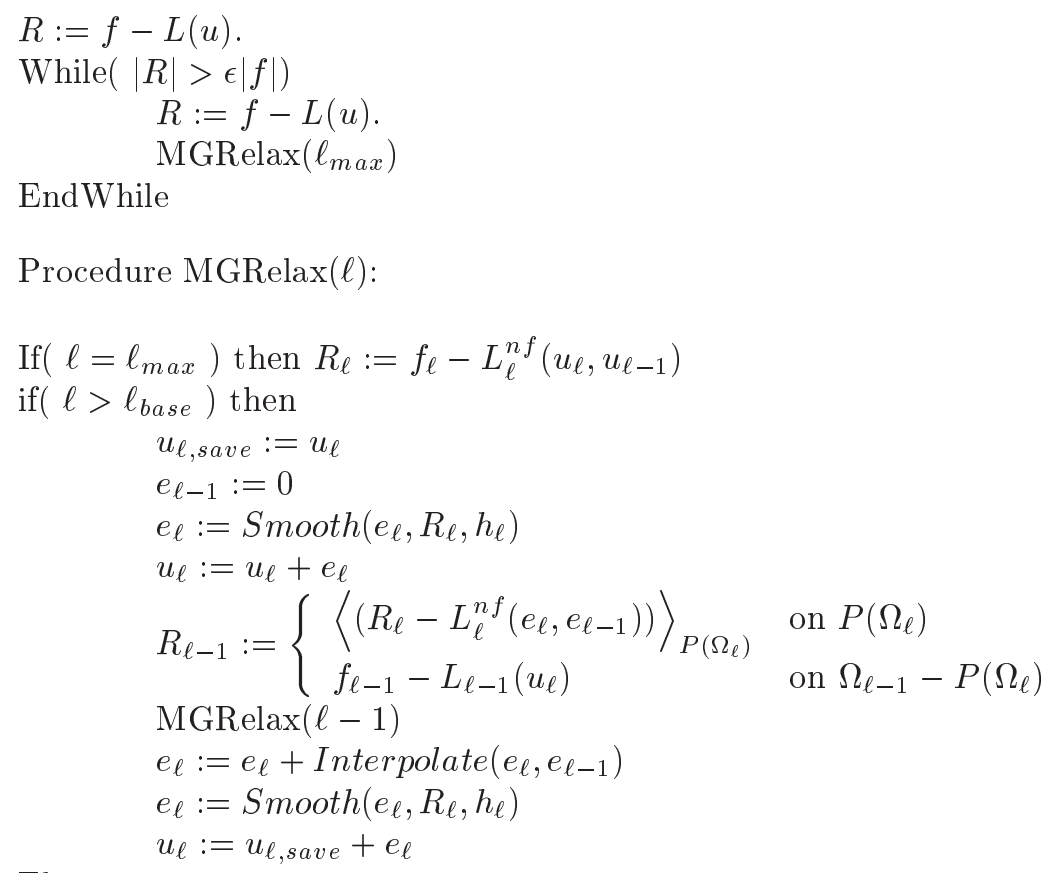

Else

EndIf

$$
\begin{aligned}
& \text { Smooth } L_{\ell_{\text {base }}}\left(e_{\ell_{\text {base }}}\right)=R_{\ell_{\text {base }}} \\
& u_{\ell_{\text {base }}}:=u_{\ell_{\text {base }}}+e_{0}
\end{aligned}
$$

Here, $L_{\ell}$ denotes the level operator $L_{\ell}^{n f}$ restricted to $\Omega_{\ell}-P\left(\Omega_{\ell+1}\right)$ and augmented with the appropriate refluxing terms to impose the level $\ell+1$ fluxes on $I$ (the $n f$ in the superscript of the operator defined by (4.66) means "no fine", implying that there is no dependence on fine grid information). For the Smooth() function, we use a single red-black Gauss-Seidel relaxation sweep, and for the Interpolate() step we use piecewise-constant interpolation, enforcing the boundary condition $e_{\ell}=Q\left(e_{\ell}, e_{\ell-1}\right)$ at the interface between levels $\ell$ and $\ell-1$.

Using this multilevel composite solution algorithm, we anticipate effective convergence rates typical of multigrid methods for elliptic problems. Our definition of "effective" in this context is nominally an order of magnitude reduction in the residual for each cycle over the level hierarchy. The convergence of the algorithm depends significantly upon the behavior of the level operator coefficients 
$a_{\ell}$ and $b_{\ell}$ in (4.66), and especially the relationship of these coefficients on $P\left(\Omega_{\ell+1}\right)$ to $a_{\ell+1}$ and $b_{\ell+1}$. If these coefficients are smoothly varying over the problem domain, a simple arithmetic averaging of $a_{\ell+1}$ and $b_{\ell+1}$ to obtain coarsened $a_{\ell}$ and $b_{\ell}$ on $P\left(\Omega_{\ell+1}\right)$ is sufficient to obtain an effective convergence rate. This is indeed the case for the solution of the composite temperature synchronization system (4.61)-(4.63). However, in the composite Newton solution of the potential synchronization system (4.40)-(4.44), the linearization of the boundary condition (3.40) results in Jacobian coefficients $a_{\ell}$ and $b_{\ell}$ that vary by a few orders of magnitude at the physical boundary. For such coefficients, arithmetic averaging of the $b_{\ell}$ coefficients fails to yield an adequate coarsening. If, instead, the coefficients $b_{\ell}$ are harmonically averaged in the coordinate directions normal to their respective cell edges, we again obtain good convergence rates. We have also found it generally advantageous to "W-cycle" the multilevel algorithm, i.e., visit each refinement level twice before returning to the next finer level. This biases the computational work towards the coarser levels, which tends to improve both the robustness and convergence rate of the iteration, although the work per cycle is increased.

The cost of the composite synchronization steps relative to the sum of the integration and sychronization times depends upon a number of factors. For problems like those for which results are reported in Chapters 6 and 7, this ratio is typically about 20-30\% in our current software implementation. 


\section{Chapter 5}

\section{Analysis of the Method}

The algorithm described above has been implemented in the Adaptive Plasma Model (APM) computer code. APM is a hybrid $\mathrm{C}++$ /FORTRAN code built upon an object oriented adaptive mesh refinement framework [20]. The code currently runs on a DEC Alpha computer.

\subsection{Uniform Grid Analysis}

This section presents computational and analytic results which help assess the performance of the single grid algorithm in terms of accuracy and efficiency. First, the single grid algorithm is compared to the INDUCT [63] code and differences are discussed. Numerical results which assess the spatial order of accuracy, for the single grid case, are presented. Analysis of the adaptive grid algorithm is discussed in the next section.

\subsubsection{Comparison to INDUCT}

The INDUCT code has been used in the semiconductor processing industry and has been benchmarked against experiment, $[12,63,65]$. The governing equations used by APM are very similar to those used by INDUCT. INDUCT includes many additional features and capabilities 


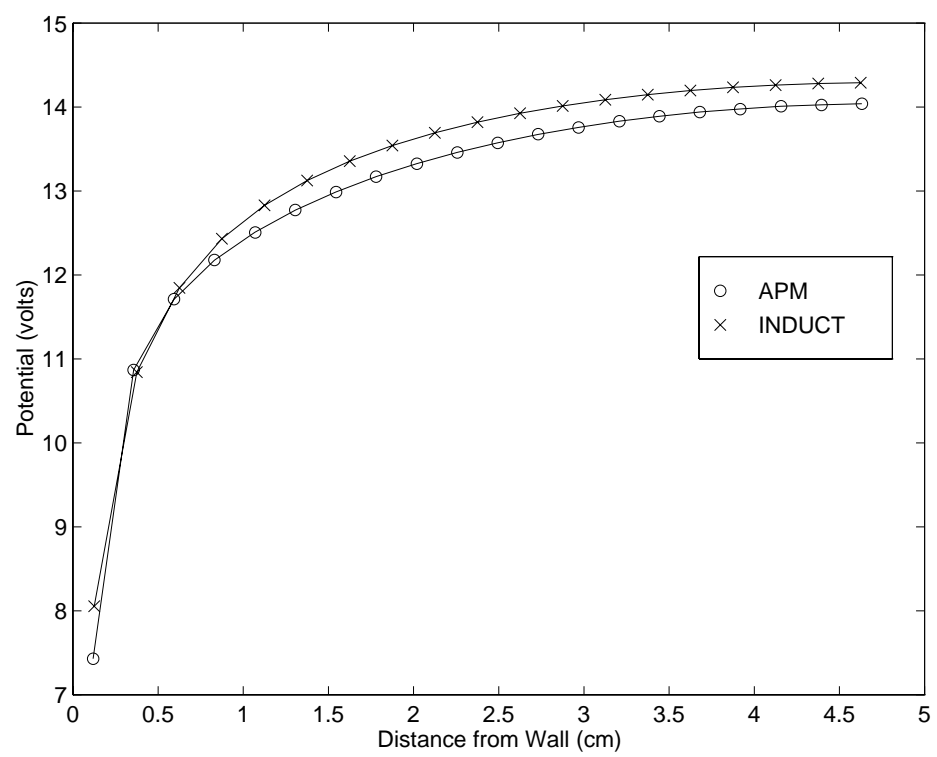

Figure 5.1: Potential profiles for APM and INDUCT test case.

such as neutral flow, an RF coil model coupled to the plasma, multiple ion species with variable temperature, and complex chemistry. For this comparison, these features were not used.

A difficulty in comparing the codes is the individual coordinate systems. APM and INDUCT are both two-dimensional codes but INDUCT uses cylindrical coordinates and APM uses Cartesian coordinates. A one-dimensional comparison can be made, however, if the cylindrical case has a very large aspect ratio $(Z$ scale $\ll R s c a l e)$ and the solution on the centerline is used. This can then be compared to a Cartesian calculation with suitably chosen symmetry boundary conditions. This was done and the results are shown in Figures 5.1-5.4. The simulation length was $4.75 \mathrm{~cm}$, half of a $9.5 \mathrm{~cm}$ region with a symmetry boundary. The neutral background gas was assumed to be Argon at approximately 60 mTorr. Ionization and elastic collision data was obtained by interpolation from the same tabular data in both codes [25]. The grid spacing was $0.25 \mathrm{~cm}$ and the calculations were run to $500 \mu$ s at which time both codes were in steady state. The inductive power was a uniformly distributed 0.2368 watts $/ \mathrm{cm}^{3}$. The APM calculation started with constant electron and ion density profiles of $10^{11} \mathrm{~cm}^{-3}$ and zero particle velocities. The initial electron temperature was $2.0 \mathrm{eV}$ and 


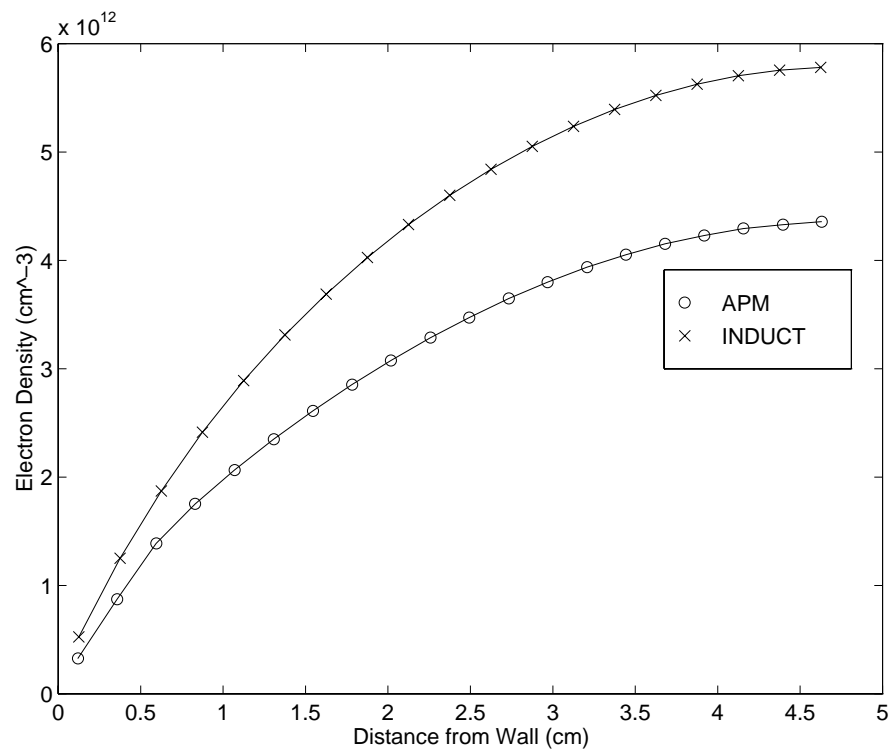

Figure 5.2: Electron density profiles for APM and INDUCT test case.

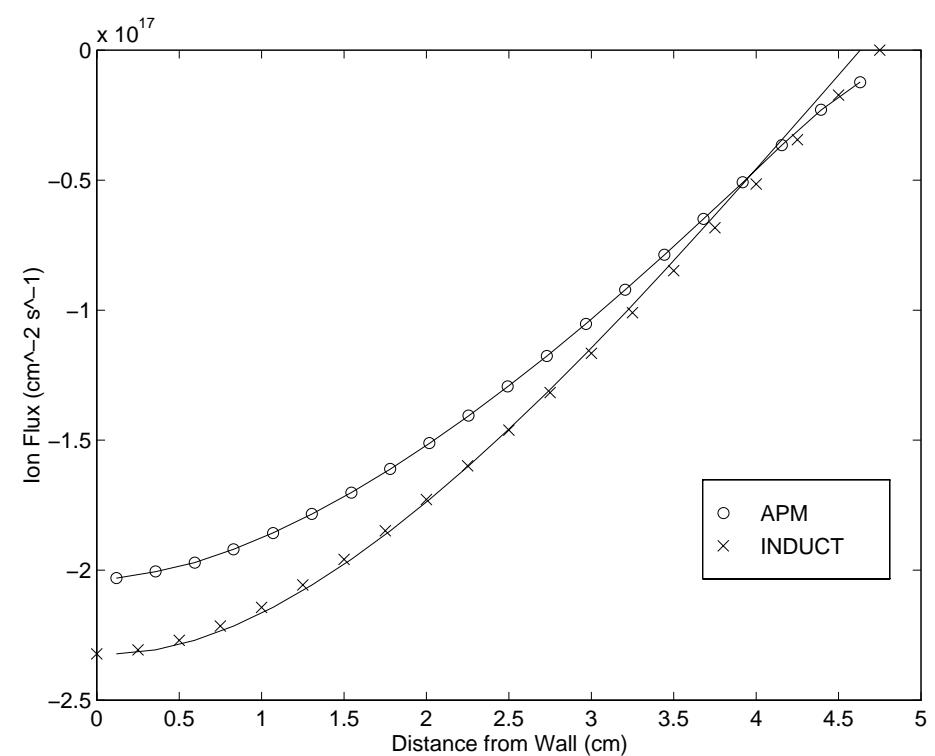

Figure 5.3: Ion flux profiles for APM and INDUCT test case. 


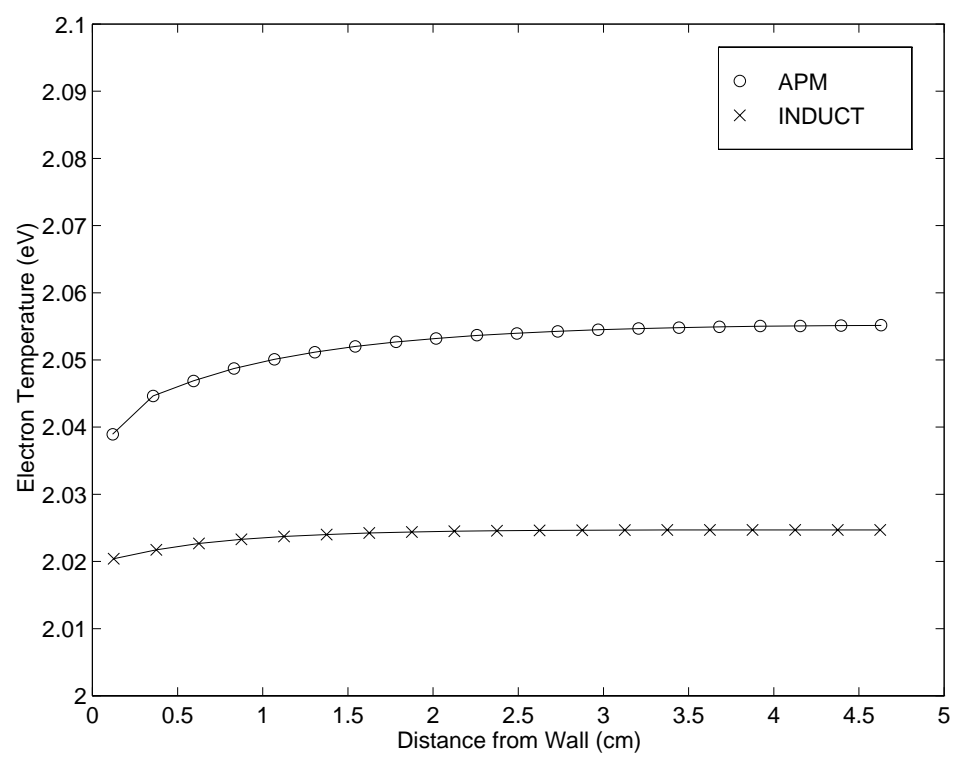

Figure 5.4: Electron temperature profiles for APM and INDUCT test case.

the constant ion and neutral temperatures were $0.05 \mathrm{eV}$.

The results generally agree, but the differences are large enough to deserve consideration. These differences can be attributed to a number of factors. The two codes use different discretizations, the electron and ion advection procedures are particularly dissimilar. First-order upwind differencing was used within INDUCT while a second-order Godunov method was used within APM. The potential boundary conditions were also slightly different. The INDUCT calculation used a cell-centered, boundary while the APM boundaries are edge centered. Considering these differences, we consider the agreement between the codes to be reasonable.

\subsubsection{Convergence of the Single Grid Algorithm}

The single grid algorithm described in Chapter 3 is a complex time splitting which allows us to solve a coupled set of non-linear partial differential equations. Despite the difficulty solving such a system, we have endeavored to retain second order spatial accuracy. The use of the secondorder Godunov method for ion and electron advection and extrapolated electric fields at physical 
boundaries illustrate this. Nevertheless, we would like to know to what extent we have succeeded in retaining high accuracy. This section addresses this issue by describing calculations and an analysis of the results which numerically approximate the order of the local truncation error.

If we look at the computed solution for some quantity in one dimension (electron density, ion flux, etc.) we can write it as :

$$
\phi(x)=E(x)+\alpha(x) h^{n}+\beta(x) h^{n+1}+\cdots
$$

where $\phi(x)$ is the computed solution, $E(x)$ is the exact solution, $h$ is the grid spacing and $\alpha, \beta$, etc. are unknown coefficients and $n$ represents the order of the leading term of the truncation error. If the leading term is dominant and the coefficient is independent of $h$, we can approximate this relation by

$$
\phi(x) \approx E(x)+\alpha(x) h^{n} .
$$

Consider three similar calculations which differ only in cell size. If the temporal errors are negligible, then each of these calculated solutions may be represented by the relation (5.2). If the calculations use cell sizes $4 h, 2 h$ and $h$, with corresponding computed solutions $\phi_{h}(x), \phi_{2 h}(x)$ and $\phi_{4 h}(x)$, we can estimate the value of $n$ and, hence, the order of the method. First, we have

$$
\begin{aligned}
\phi_{4 h}(x) & \approx E(x)+\alpha(x)(4 h)^{n} \\
\phi_{2 h}(x) & \approx E(x)+\alpha(x)(2 h)^{n} \\
\phi_{h}(x) & \approx E(x)+\alpha(x)(h)^{n}
\end{aligned}
$$

If we then subtract (5.4) from (5.3), subtract (5.5) from (5.4), and take ratios we see

$$
2^{n} \approx \frac{\phi_{4 h}(x)-\phi_{2 h}(x)}{\phi_{2 h}(x)-\phi_{h}(x)}
$$




$$
n \approx \log \left(\frac{\phi_{4 h}(x)-\phi_{2 h}(x)}{\phi_{2 h}(x)-\phi_{h}(x)}\right) / \log (2)
$$

Three calculations were performed which differed in the number of grid points used $(256$, 512 and 1024, respectively). Each calculation was run with a timestep sufficiently small to make temporal errors negligible. This was accomplished by running a sequence of calculations with progressively smaller timesteps, until the solution converged. Because of the computational difficulty running the finest of these calculations, they were only run to $0.1 \mathrm{~ns}$. During this time, only part of the plasma has evolved from the original state. In particular, the electron density, potential and ion flux have time to evolve (at least near the boundary), but the ion density and electron temperature have not. Therefore, these variables are the only ones considered. The computation (5.7) was performed for these variables and results are shown in Figures 5.5, 5.6 and 5.7. The variation in these calculations suggest we are not yet in the asymptotic regime assumed in (5.2). Nevertheless, these suggest that the potential, electron density, and ion flux are certainly being calculated superlinearly and probably to second order. We would like to have a similar result for the ion density, but the ion advection has been shown in [17] to be second order so there is less concern about this quantity. We cannot say much about the electron temperature except that is rarely shows much spatial variation in any case.

\subsection{Locally Refined Grid Analysis}

In this section we present numerical results which will help assess the utility of the previously described locally refined algorithm. The accuracy of APM on locally refined grids is analyzed by comparisons to uniform grid calculations and we investigate the effects of different two-dimensional grid structures on accuracy. The efficiency of the code is illustrated with a comparison of timings and memory requirements for refined grid and equivalent uniform grid calculations. The effect of various multilevel timestep selection schemes is examined for accuracy and efficiency. We then discuss criteria for adaptive refinement and finally we show the benefit of adapting the solution in time 


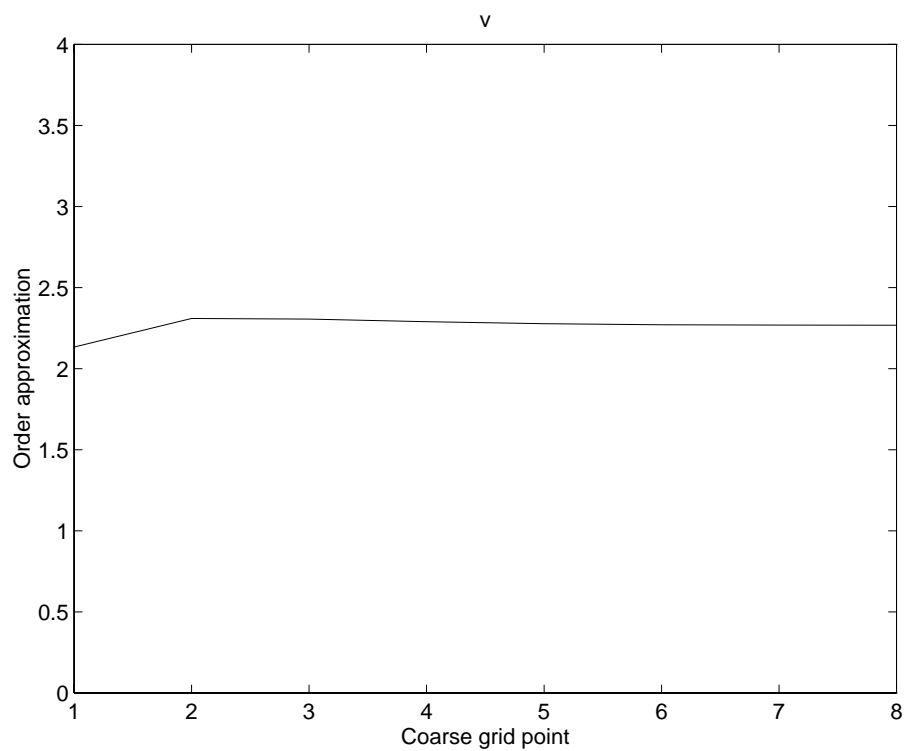

Figure 5.5: Order calculation for potential.

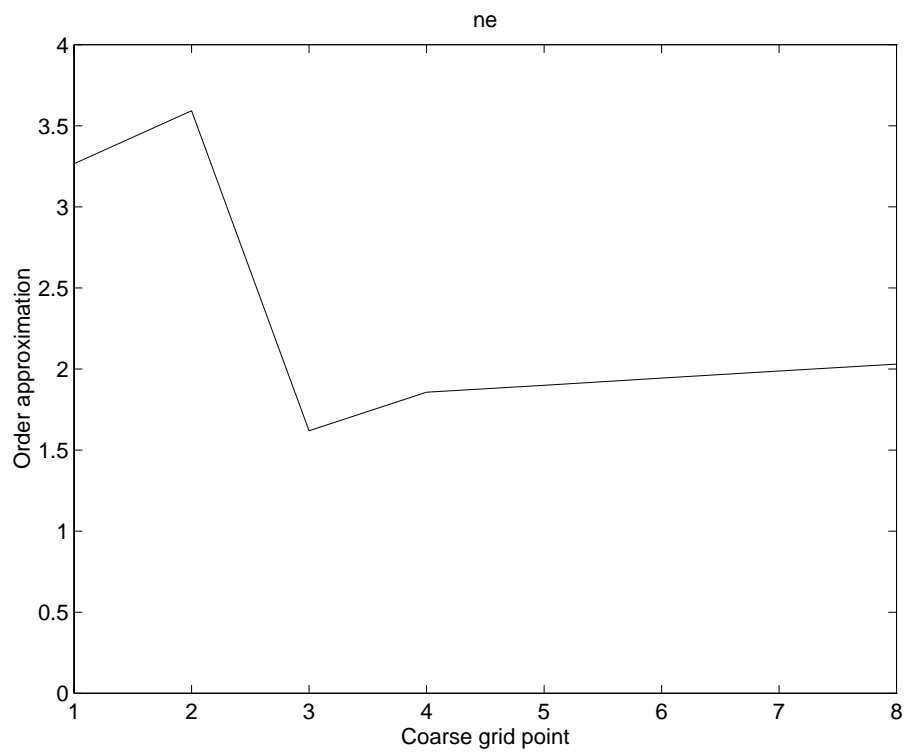

Figure 5.6: Order calculation for electron density. 


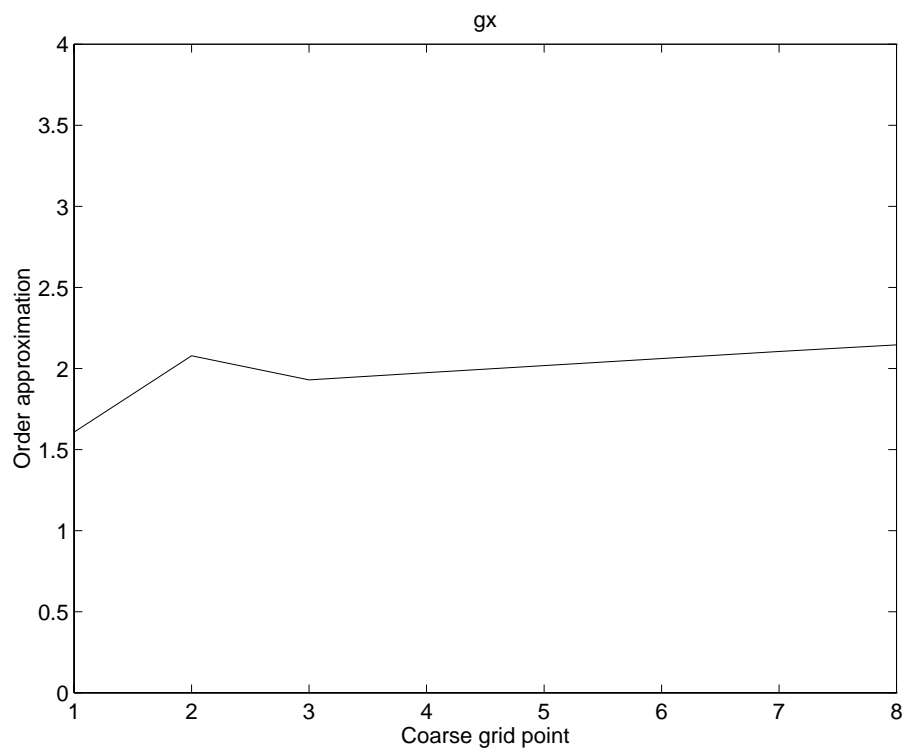

Figure 5.7: Order calculation for ion flux.

in order to efficiently obtain steady state solutions.

\subsubsection{Demonstration of Accuracy Using Locally Refined Grids}

Consider a locally refined grid calculation with cell sizes $h_{\ell}, \ell=0, \ldots, \ell_{\max }$, where $h_{\ell_{\max }}$ is the cell size corresponding to the finest cells and $h_{0}$ corresponding to the coarsest cells. Such a calculation cannot be expected to be more accurate than a uniform grid calculation with cell size $h_{\ell_{\max }}$. In fact, the degree to which the locally refined calculation matches the fine, uniform grid calculation is a measure of its accuracy.

Figures 5.8 through 5.11 show a comparison of potential, ion flux, electron density and electron temperature profiles using a locally refined grid, a fine uniform grid and a coarse uniform grid. The calculation used argon with a neutral density of approximately 60mTorr and a uniform power density of 0.1 watts $/ \mathrm{cm}^{3}$. Symmetry boundary conditions on the top and bottom of the rectangular domain restrict the solution variation to the $\mathrm{x}$-direction. The uniform fine grid spacing corresponds to the finest cells in the refined grid and the uniform coarse grid spacing corresponds to 


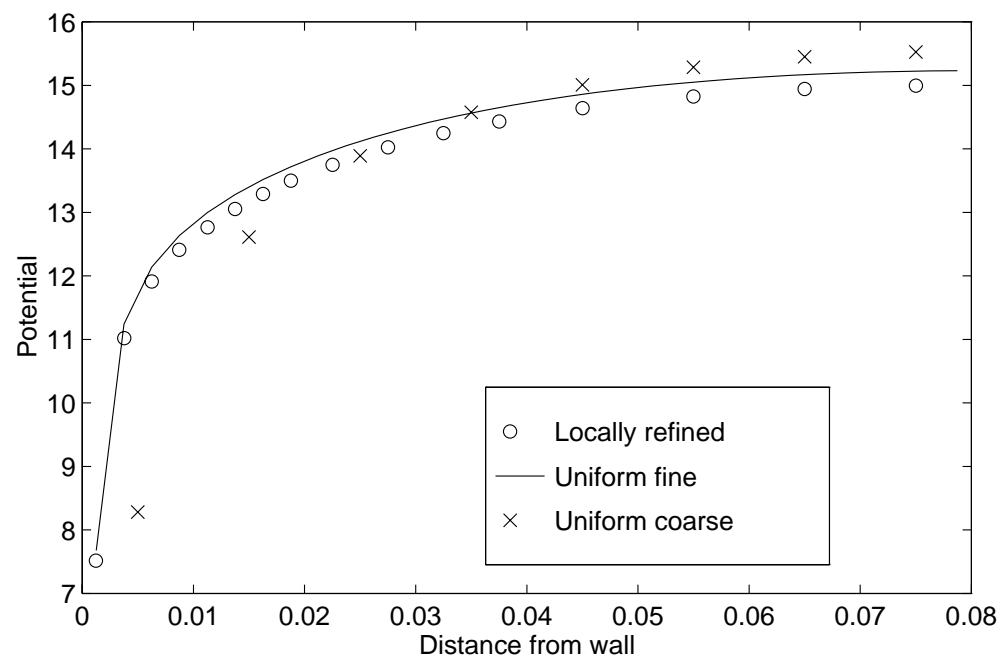

Figure 5.8: Potential profile for locally refined, fine and coarse grid calculations.

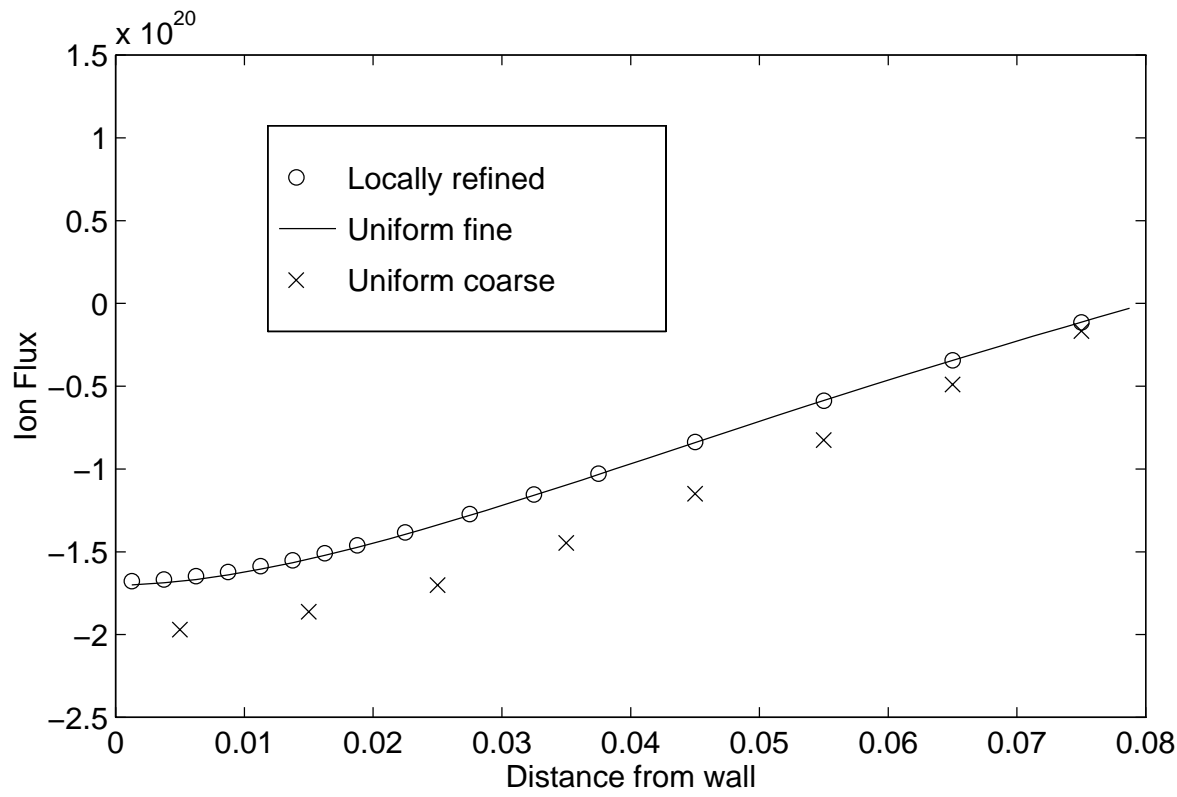

Figure 5.9: Ion flux profile for locally refined, fine and coarse grid calculations. 


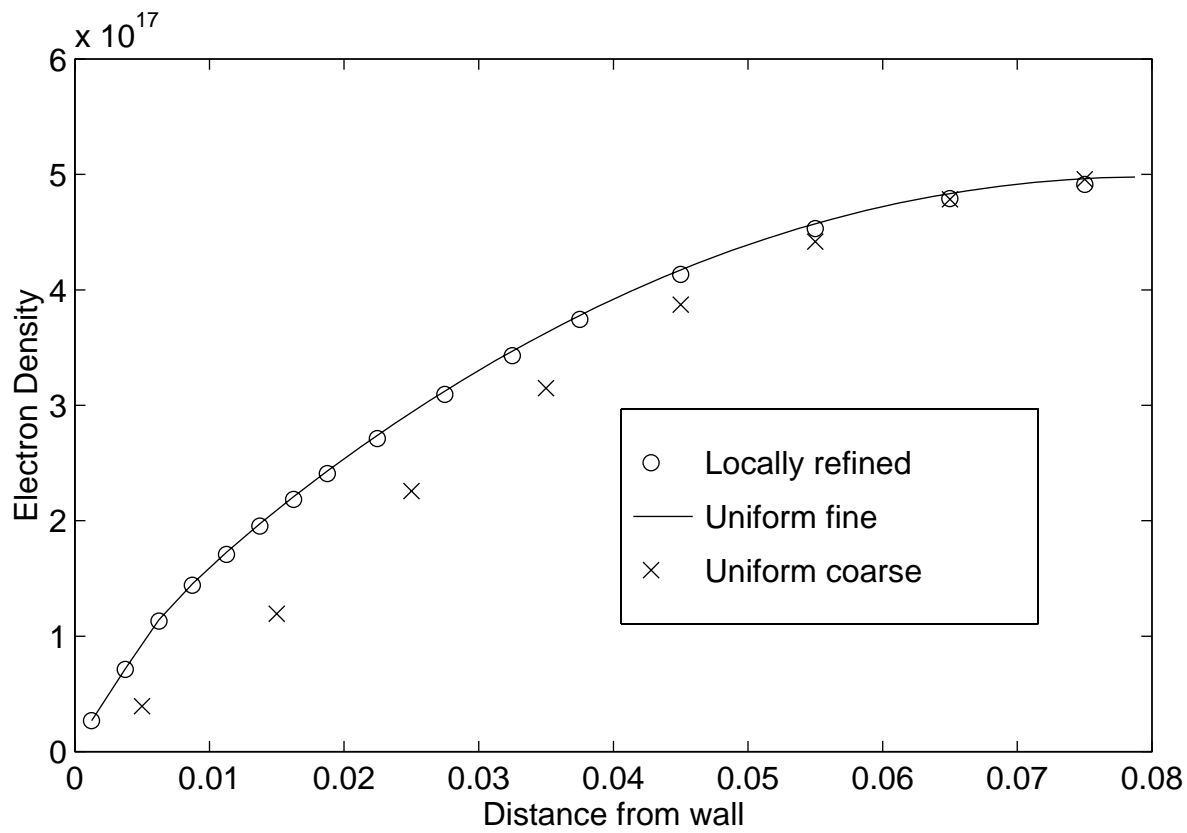

Figure 5.10: Electron density profile for locally refined, fine and coarse grid calculations.

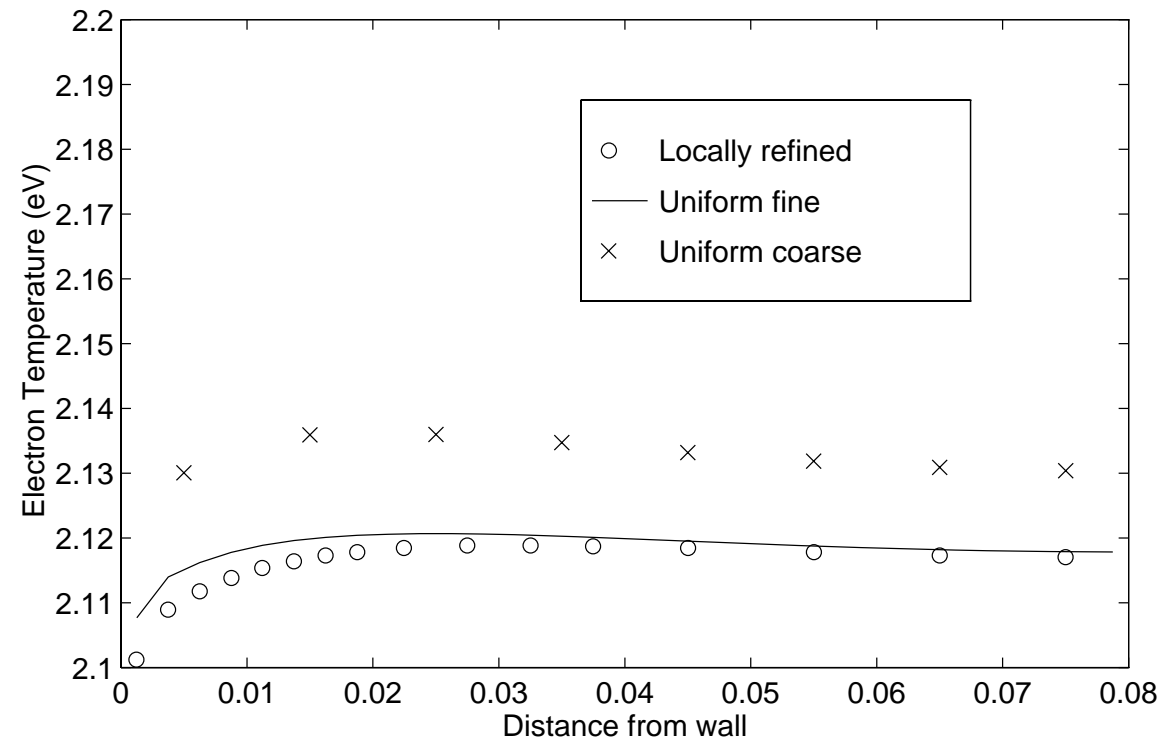

Figure 5.11: Electron temperature profile for locally refined, fine and coarse grid calculations. 


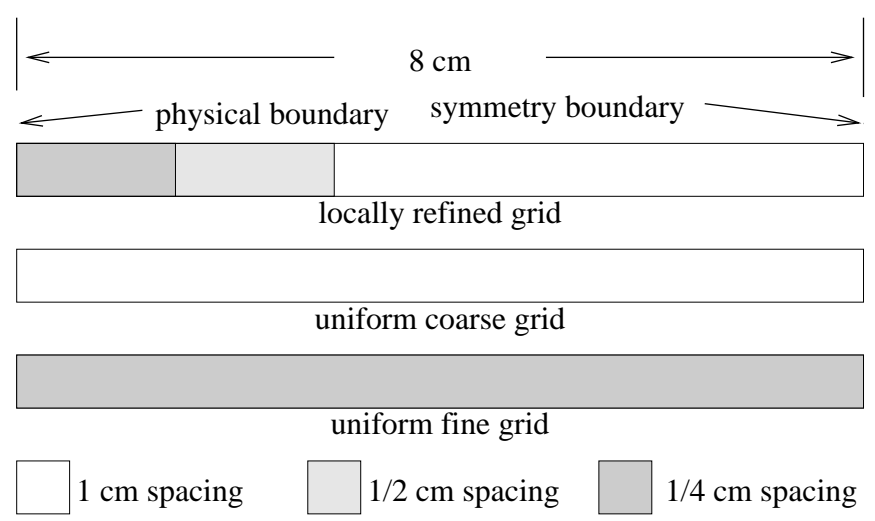

Figure 5.12: Locally refined, uniform fine and uniform coarse grids used to generate results shown in Figures 5.8 through 5.11

the coarsest cells. The refined grid uses 3 levels. Each level was generated by refining the leftmost 3 cells of the next coarser level by a factor of 2. This grid structure is shown in Figure 5.12. The agreement in the fine portion of the locally refined grid is excellent while the agreement in the coarse region is much better than the equivalent coarse grid.

The accuracy of the locally refined calculation depends critically on where the refined grids are used. The high accuracy of this calculation is due in large part to the placement of the fine grids near the boundary. As expected this region has the most variation in potential, density and charge. Consequently, smaller cells are required to resolve this variation. Conversely, the use of coarse grids has little effect on the quasi-neutral region in the center of the plasma. We investigate the relationship between grid placement and accuracy in the next section. Most of this work has used heuristically placed grids, but the code is capable of choosing grids to refine based on values of the state variables or their gradients. These issues are also discussed in a later section.

\subsubsection{Effect of Grid Selection on Accuracy}

The previous section contained a comparison of a locally refined calculation with two uniform calculations of different resolutions. The good agreement with the finer of these uniform grids serves as a demonstration that, at least in this case, local refinement is performing as intended. 
In this section we look at additional calculations in a more quantitative way. In particular the effects of different grids (different resolutions, levels of refinement, etc.) on the solution accuracy are examined.

The first part of this section compares 3 different locally refined calculations with the same peak resolution and fine grid structure to uniform grid calculations with different resolutions. The second compares a number of two level calculations in which we vary the fraction of the coarse grid which is refined.

\section{Solution dependence on number of levels and refinement ratios.}

Three uniform grid calculations were performed along with three locally refined calculations. Each was run to a final simulation time of $10 \mu \mathrm{s}$. The locally refined calculations differed in the number of levels used, the resolution of the base level and the refinement ratios on each level. In each case, however, the same grid structure was used on the finest level. The problem domain and boundary conditions are the same as that shown in Figure 5.35. The individual grid structures are shown in Figure 5.13.

The results were compared in two ways. First, We define a normalized $L_{2}$ norm of the composite solution $f$ as

$$
\|f\|_{L_{2}} \equiv \sqrt{\frac{\sum_{\text {cells }}\left(<f>-<f_{\text {uniform }}>\right)^{2}}{\sum_{\text {cells }}<f_{\text {uniform }}>^{2}}}
$$

where $f_{\text {uniform }}$ is the uniform fine grid solution and volume weighted averaging to the coarsest grid is denoted by the operator $\langle\cdot\rangle$.

This norm was calculated for all state variables as well as the charge. The errors are with respect to the highest resolution, uniform grid solution (32x32 cells). Only one component of ion flux is presented, however, since it is the same as the other in this symmetric problem. For the $\mathrm{L}_{2}$ norm calculation, the fine calculations are averaged down to the coarsest uniform grid resolution for comparison. This is appropriate for conserved variables $\left(n_{e}, n_{i},{ }_{i}\right.$, etc. $)$ but only a figure of merit 
for $\phi$ and $T_{e}$. These results are shown in Figures 5.14 through 5.19. In addition to the $L_{2}$ norms, key extrema are tabulated in Table 5.1.

These results are encouraging. Each of the three locally refined calculations agreed quite well with the highest resolution uniform grid calculation. The refined calculations all performed much better than the second highest resolution uniform calculation, even though two of them used coarser grids in much of the domain. The differences in the refined calculations are interesting as well. The poorest calculation of the three was the three level case with two levels refined by a factor of 2. It was both less accurate and slower by almost a factor of 2 as compared to the two level, factor of 4 refinement case. The potential had the poorest agreement, perhaps suggesting errors due to the potential synchronization step. The uniform calculations actually ran faster because we are not yet in the regime where adaptivity provides any "gain".

These results argue for higher refinement ratios. It seems that the runtime and accuracy penalties associated with using more levels (and synchronization solves) is a more significant issue than is the refinement ratio mismatch. This is consistent with efficiency results given in a later section, but these same results suggest that as the number of levels rise the CPU time difference may drop. The accuracy trend may be the same, however. The good performance of the two level, refinement by 2 case is also interesting. Using more points at the coarser levels and limiting the number of cells at the finest level is advantagous and serves as another argument for local refinement.

\section{Solution dependence on fraction of domain which is refined.}

In the previous section we examined different numbers of refinement levels and different refinement ratios. In this section we look at three two level problems in order to examine the effects of refining different fractions of the domain. In the first of these refined calculations, the 2 exterior cells touching the physical boundary on the coarsest level are refined. The second refines the 4 exterior cells and the third refines the exterior 6 cells. These two level grids are depicted in Figure 5.20. As in the previous section they are compared to an equivalent uniform grid calculation with 


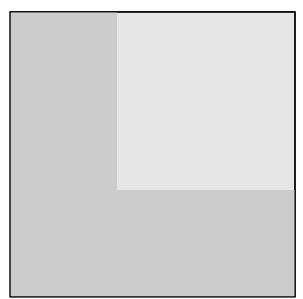

16x16 level 0 grid

1 level refined by factor of 2

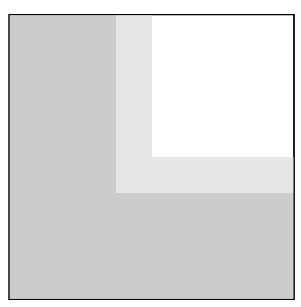

$8 \times 8$ level 0 grid

2 levels refined by factor of 2

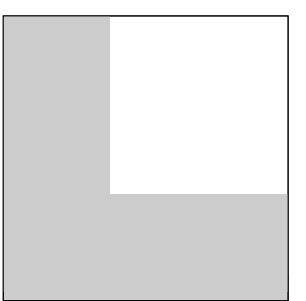

$8 \times 8$ level 0 grid

1 level refined by factor of 4

grid spacing $=0.25 \mathrm{~cm}$

grid spacing $=0.50 \mathrm{~cm}$

grid spacing $=1.00 \mathrm{~cm}$

Figure 5.13: Grids used in multilevel study of solution dependence on number of levels and refinement ratios.

\begin{tabular}{|c||c|c||c|c|c|}
\hline Level 0 grid & $16 \times 16$ & $8 \times 8$ & $16 \times 16$ & $8 \times 8$ & $8 \times 8$ \\
\hline $\begin{array}{c}\text { Refinement } \\
\text { ratio level 1 }\end{array}$ & $\mathrm{NA}$ & $\mathrm{NA}$ & 2 & 4 & 2 \\
\hline $\begin{array}{c}\text { Refinement } \\
\text { ratio level 2 }\end{array}$ & $\mathrm{NA}$ & $\mathrm{NA}$ & $\mathrm{NA}$ & $\mathrm{NA}$ & 2 \\
\hline \hline CPU time & $2.22 \mathrm{e} 3$ & $4.80 \mathrm{e} 2$ & $5.70 \mathrm{e} 4$ & $3.44 \mathrm{e} 4$ & $5.96 \mathrm{e} 4$ \\
\hline $\begin{array}{c}\text { Potential } \\
\text { (minimum) }\end{array}$ & 15.290 & 54.758 & 0.616 & 1.070 & 4.109 \\
\hline $\begin{array}{c}\text { Net charge } \\
\text { (maximum) }\end{array}$ & 74.484 & 93.153 & 0.634 & 1.244 & 3.156 \\
\hline $\begin{array}{c}\text { Ion density } \\
\text { (minimum) }\end{array}$ & 35.631 & 154.552 & 0.096 & 0.649 & 1.773 \\
\hline $\begin{array}{c}\text { Electron density } \\
\text { (minimum) }\end{array}$ & 36.538 & 156.636 & 0.099 & 0.653 & 1.827 \\
\hline $\begin{array}{c}\text { Ion flux } \\
\text { magnitude } \\
\text { (maximum) }\end{array}$ & 11.581 & 38.879 & 0.544 & 1.071 & 0.997 \\
\hline $\begin{array}{c}\text { Electron } \\
\text { temperature } \\
\text { (maximum) }\end{array}$ & 1.943 & 4.826 & 0.614 & 0.598 & 0.780 \\
\hline
\end{tabular}

Table 5.1: Errors relative to high resolution uniform grid calculation for selected quantities at $10 \mu \mathrm{s}$ (values are percent unless otherwise stated). 


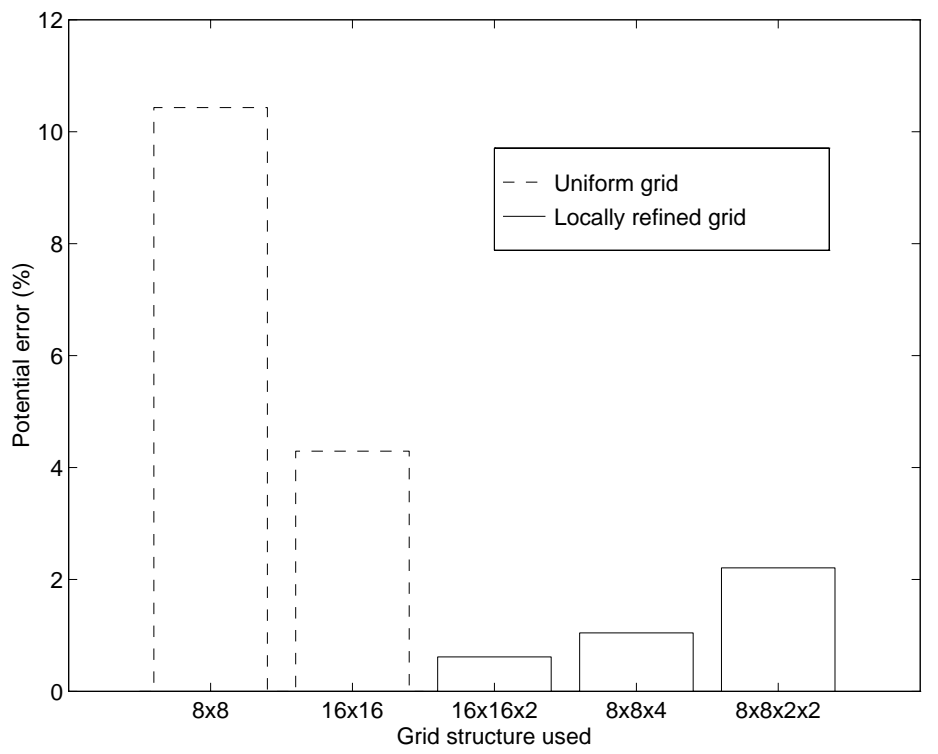

Figure 5.14: Relative error in the potential for different grid structures. The first two numbers give the number of cells in the $x$ and $y$ directions, respectively. Subsequent numbers give refinement ratios for finer levels (if they exist).

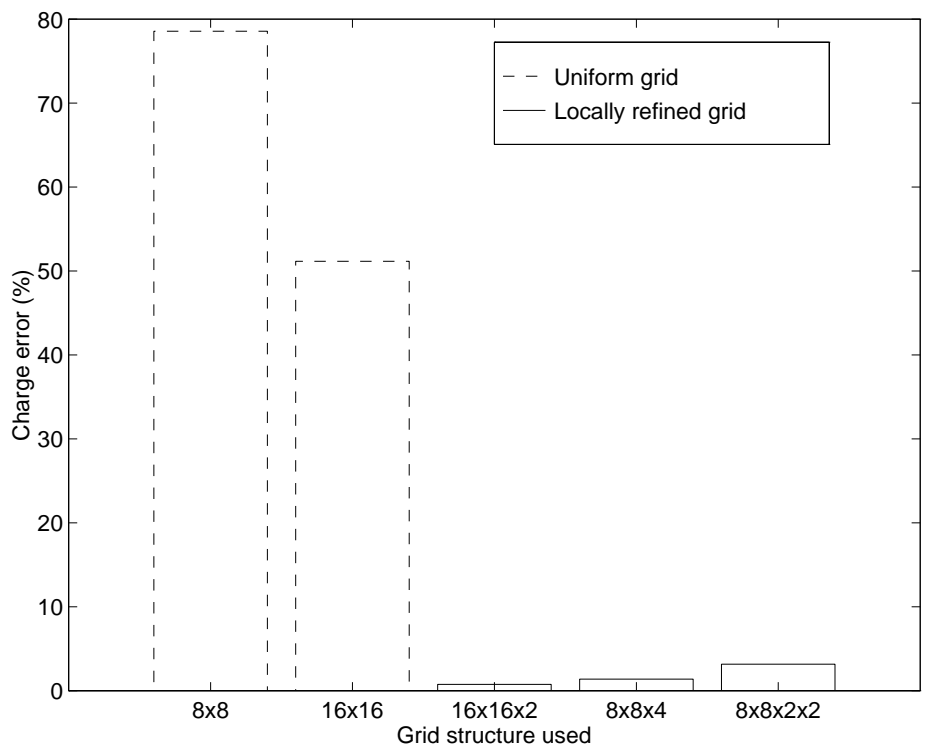

Figure 5.15: Relative error in the net charge for different grid structures. The first two numbers give the number of cells in the $x$ and $y$ directions, respectively. Subsequent numbers give refinement ratios for finer levels (if they exist). 


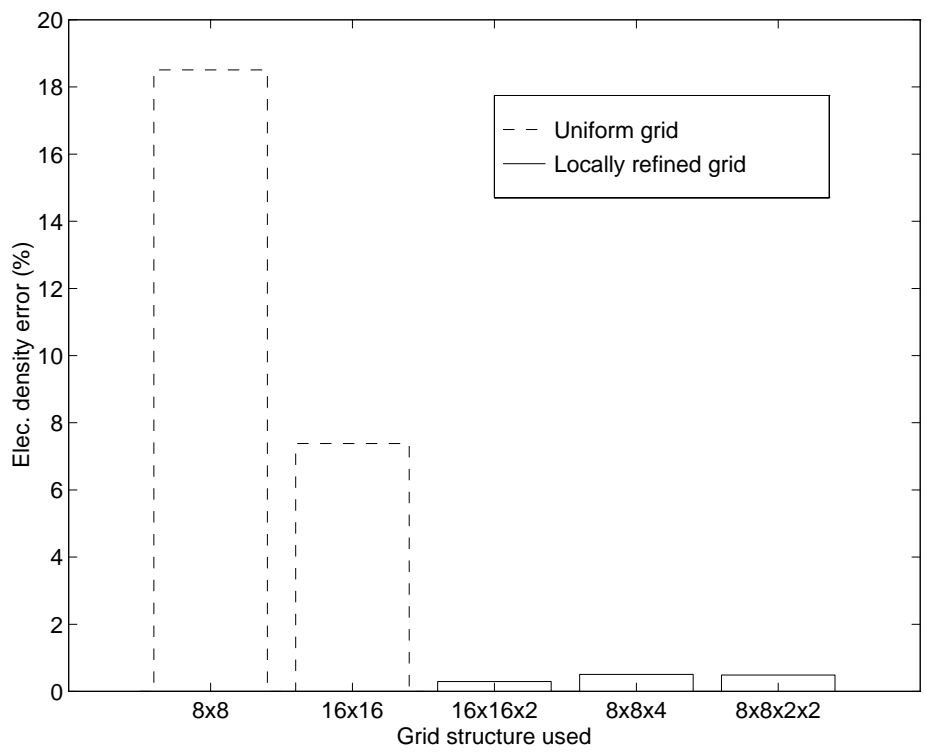

Figure 5.16: Relative error in the electron density for different grid structures. The first two numbers give the number of cells in the $x$ and $y$ directions, respectively. Subsequent numbers give refinement ratios for finer levels (if they exist).

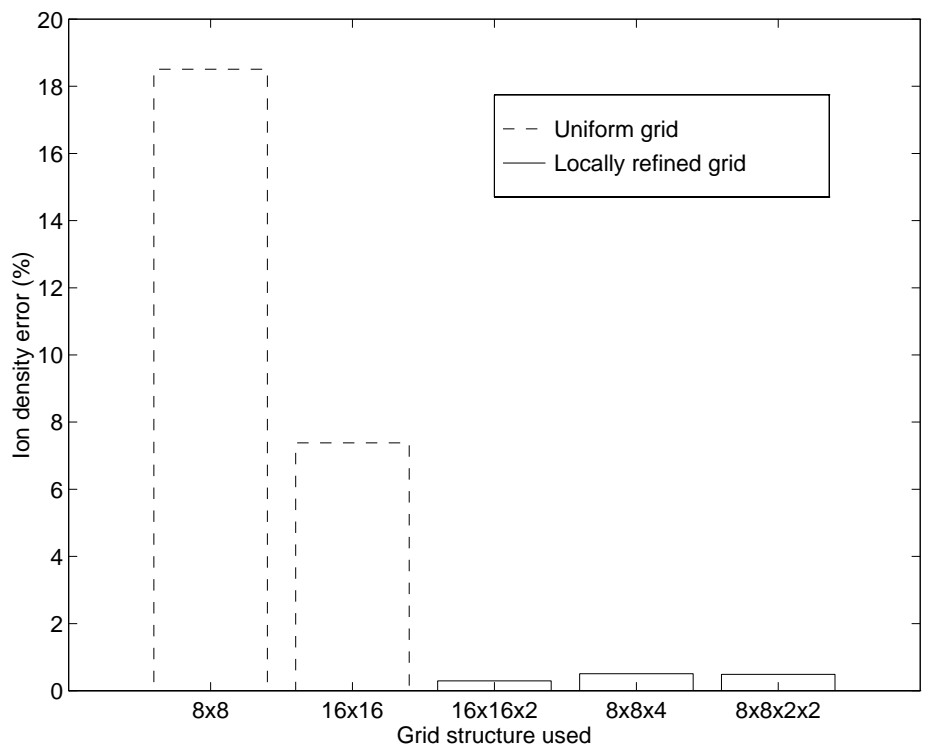

Figure 5.17: Relative error in the ion density for different grid structures. The first two numbers give the number of cells in the $x$ and $y$ directions, respectively. Subsequent numbers give refinement ratios for finer levels (if they exist). 


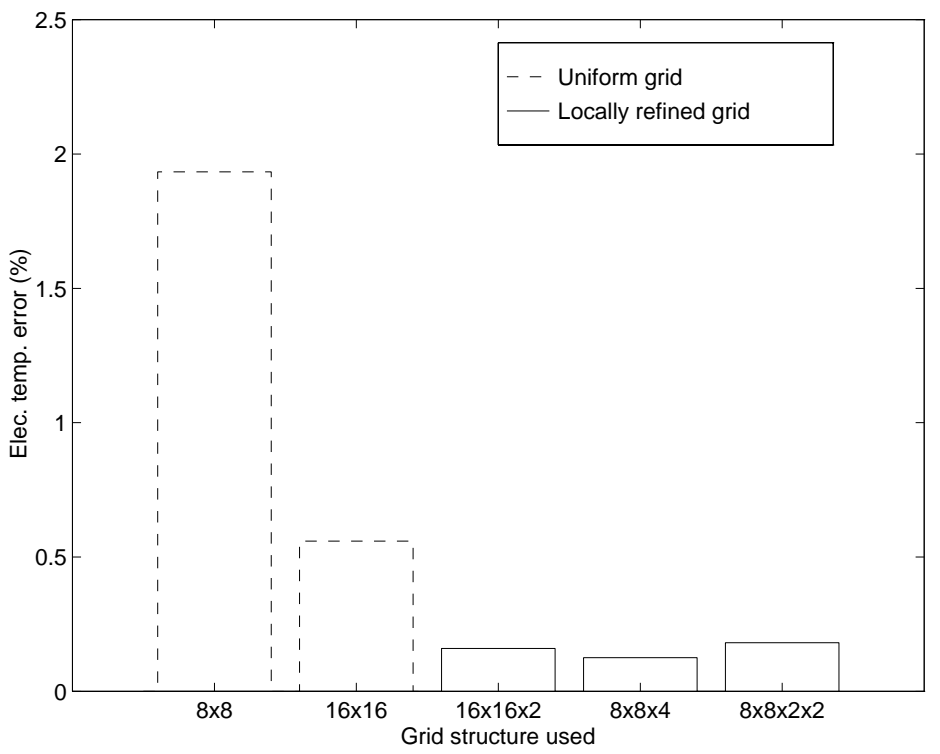

Figure 5.18: Relative error in the electron temperature for different grid structures. The first two numbers give the number of cells in the $x$ and $y$ directions, respectively. Subsequent numbers give refinement ratios for finer levels (if they exist).

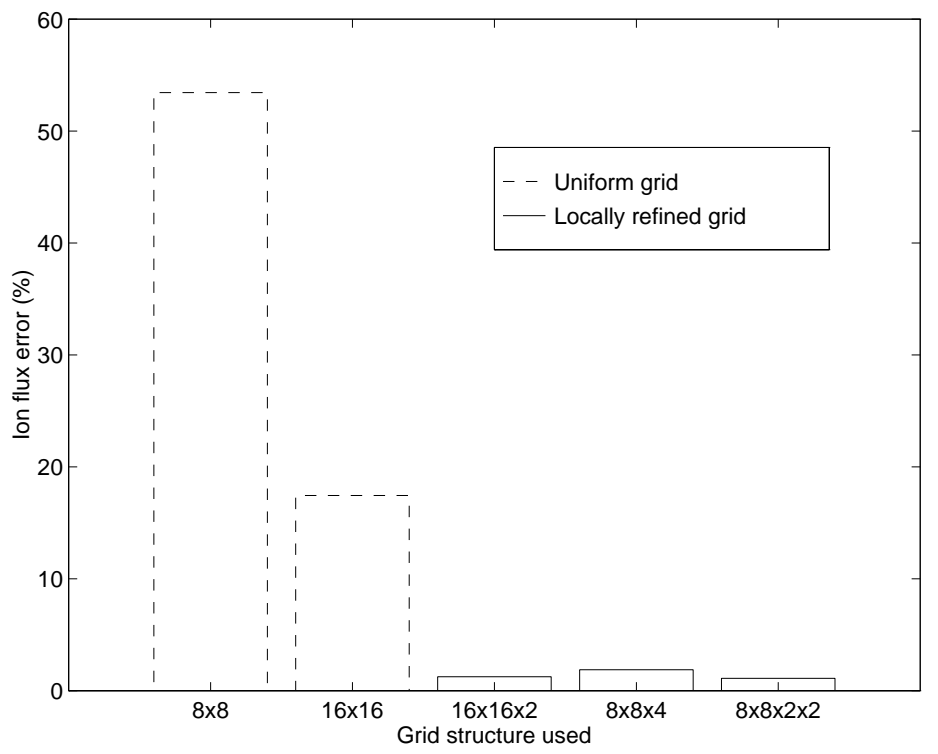

Figure 5.19: Relative error in the ion flux for different grid structures. The first two numbers give the number of cells in the $x$ and $y$ directions, respectively. Subsequent numbers give refinement ratios for finer levels (if they exist). 


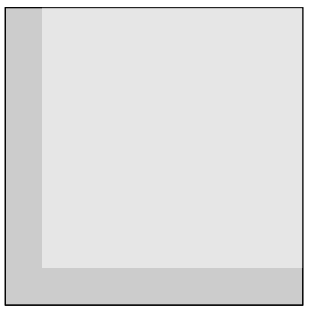

2 Exterior cells refined

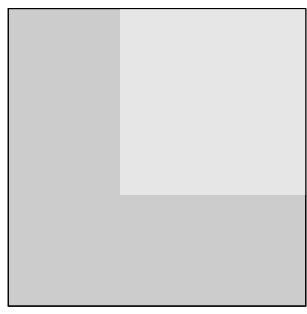

6 exterior cells refined

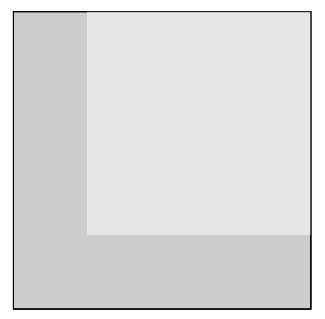

4 exterior cells refined

grid spacing $=0.25 \mathrm{~cm}$

grid spacing $=0.50 \mathrm{~cm}$

Figure 5.20: Grids used in two level accuracy study of dependence on the number of cells refined.

the same peak resolution. They are also compared to two other uniform calculations with coarser grids. The results are shown in Figures 5.21 through 5.26 for the solutions at $10 \mu$ s and in Figures 5.27 through 5.32 for the solution at $50 \mu \mathrm{s}$. Again, the relative $\mathrm{L}_{2}$ norms are used and selected quantities of interest at $10 \mu \mathrm{s}$ are shown in Table 5.2 .

In this study, the accuracy dependence on the number of refined cells at the boundary was relatively weak. The trend was also as expected, with higher accuracy (in general) in the cases using more refined cells. That this is not always the case suggests that in very smooth regions, the coarse/fine synchronization errors are comparable to coarse grid errors. These exceptions to the general trend occured for potential and temperature, both of which require synchronization solves, supporting this hypothesis. The differences were not great, however, and there was a corresponding CPU time penalty. While interesting trends could be spotted in the previous section for different refinement ratios and refined levels, that is not the case here. At least in this case, provided the sheath region is included in the level, the fraction of domain which is refined does not seem critical. 


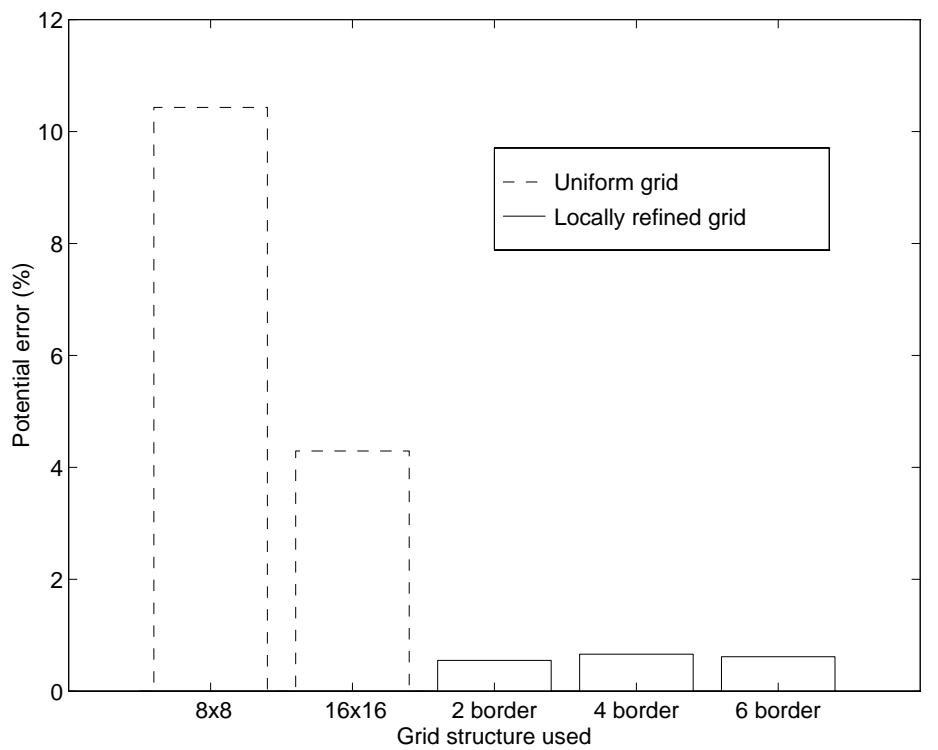

Figure 5.21: Relative error in the potential at $10 \mu$ s for different refined regions. The labels refer to the number of cells refined near the boundary.

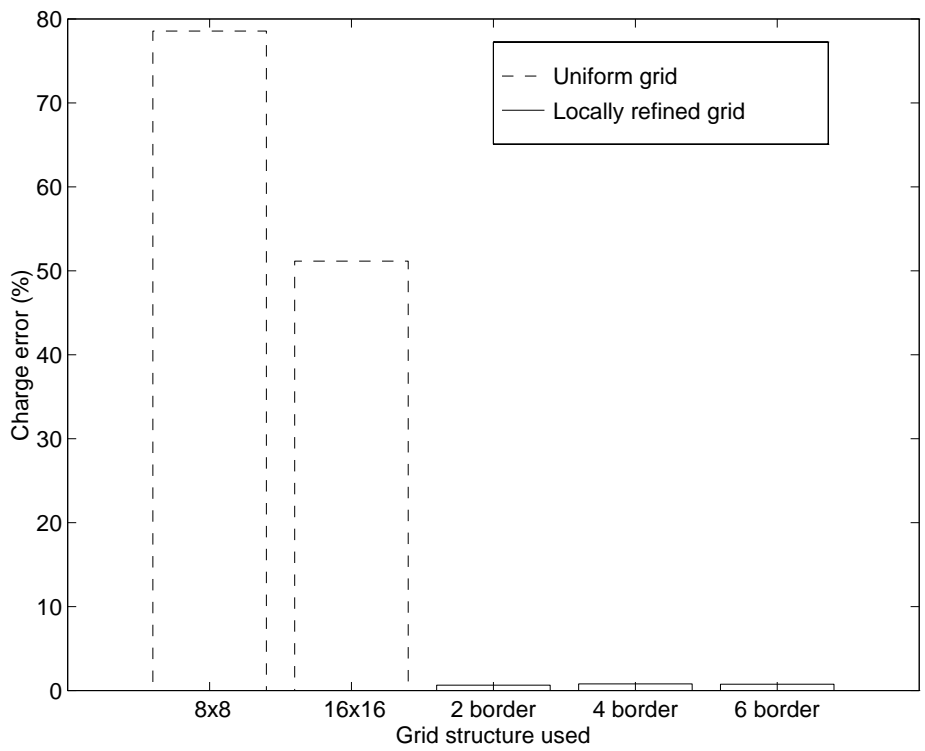

Figure 5.22: Relative error in the net charge at $10 \mu \mathrm{s}$ for different refined regions. The labels refer to the number of cells refined near the boundary. 


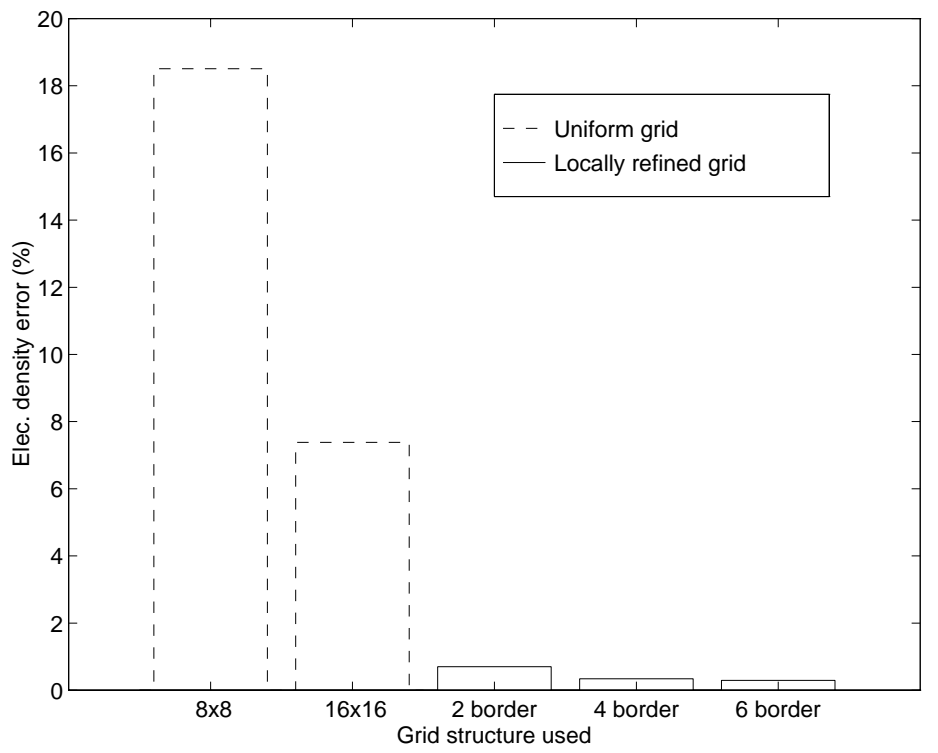

Figure 5.23: Relative error in the electron density at $10 \mu$ s for different refined regions. The labels refer to the number of cells refined near the boundary.

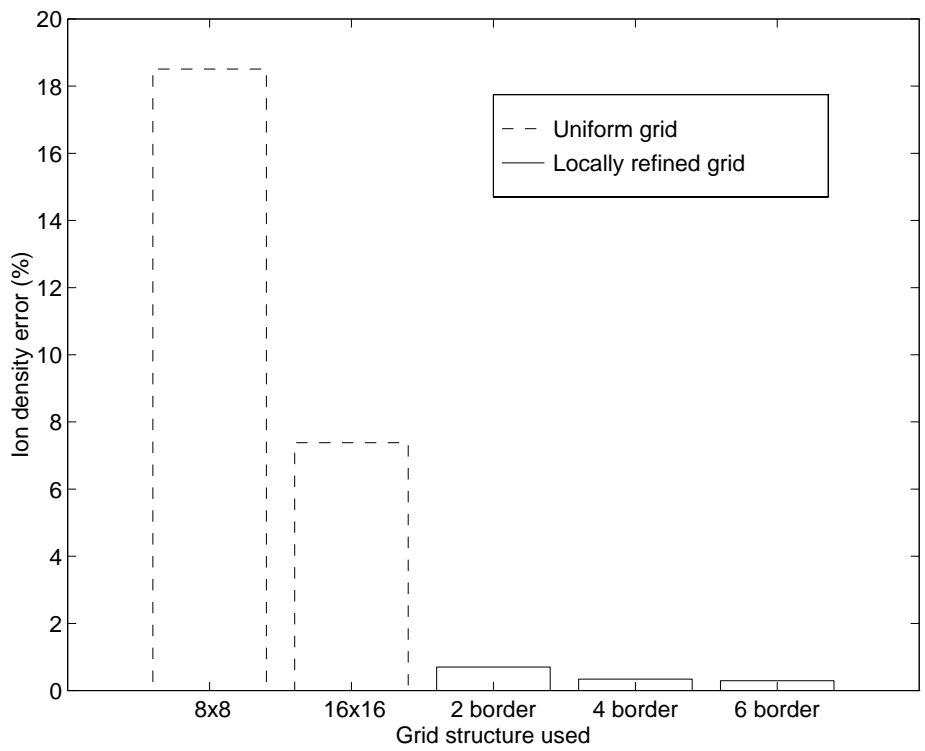

Figure 5.24: Relative error in the ion density at $10 \mu$ s for different refined regions. The labels refer to the number of cells refined near the boundary. 


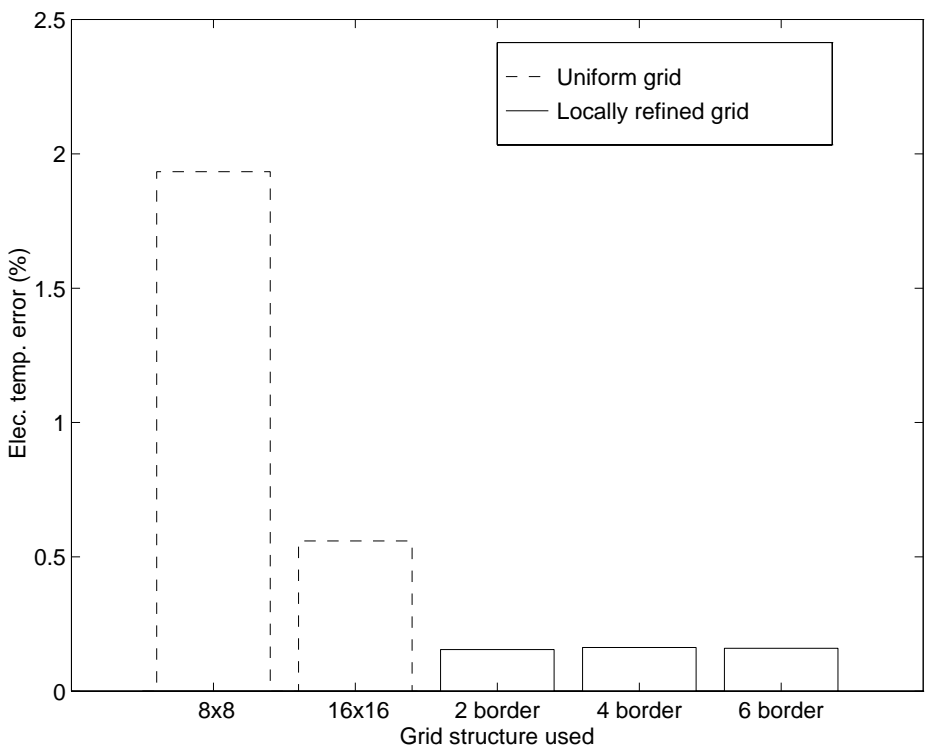

Figure 5.25: Relative error in the electron temperature at $10 \mu \mathrm{s}$ for different refined regions. The labels refer to the number of cells refined near the boundary.

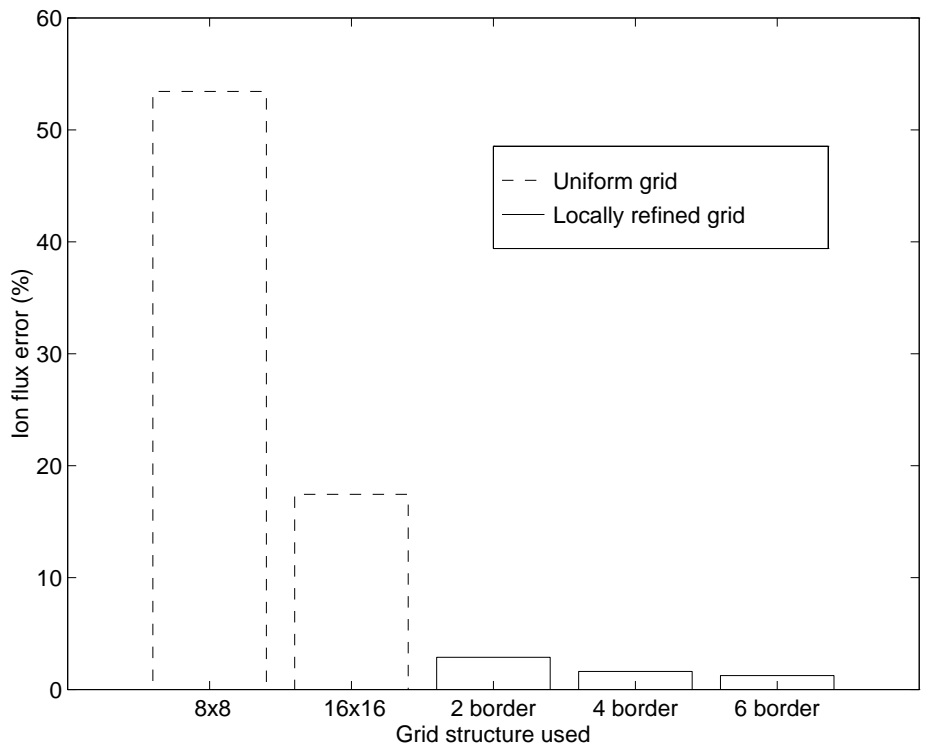

Figure 5.26: Relative error in the ion flux at $10 \mu$ s for different refined regions. The labels refer to the number of cells refined near the boundary. 


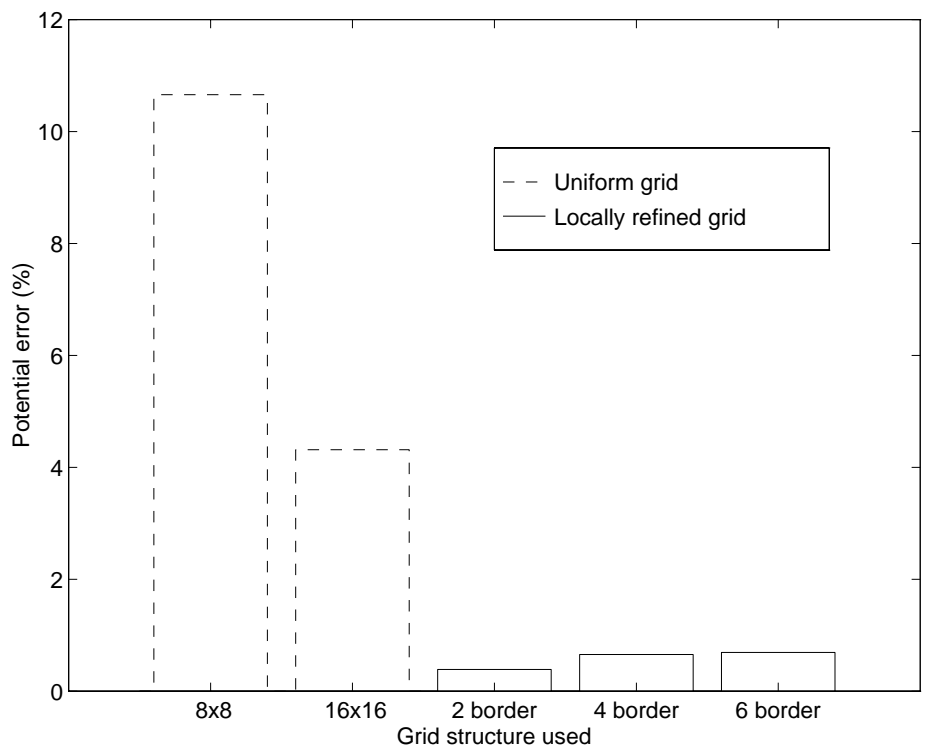

Figure 5.27: Relative error in the potential at $50 \mu$ s for different refined regions. The labels refer to the number of cells refined near the boundary.

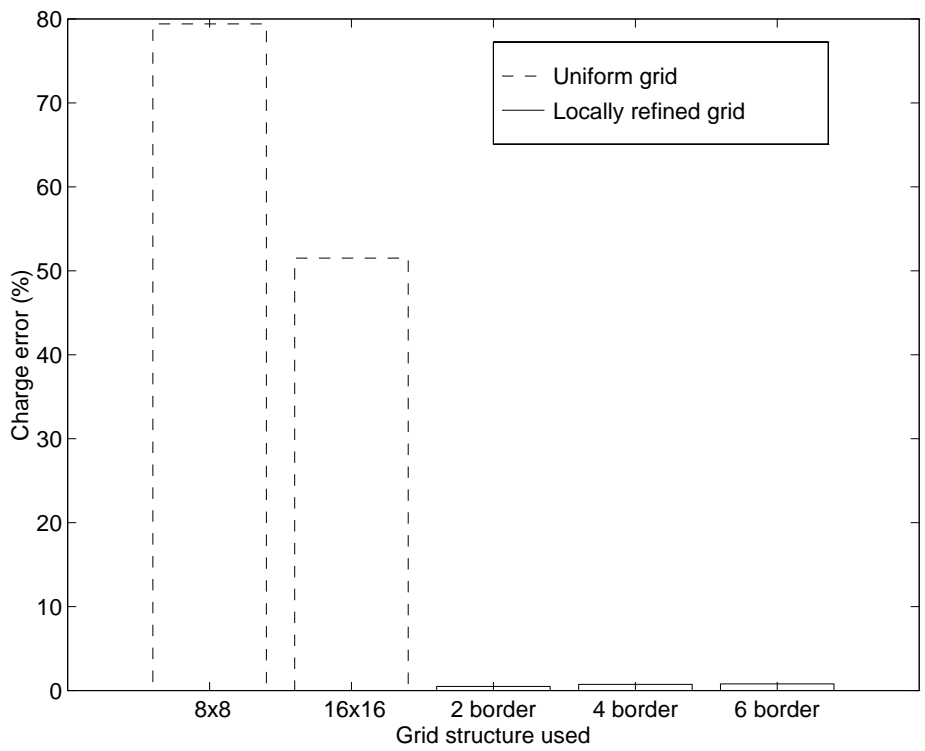

Figure 5.28: Relative error in the net charge at $50 \mu \mathrm{s}$ for different refined regions. The labels refer to the number of cells refined near the boundary. 


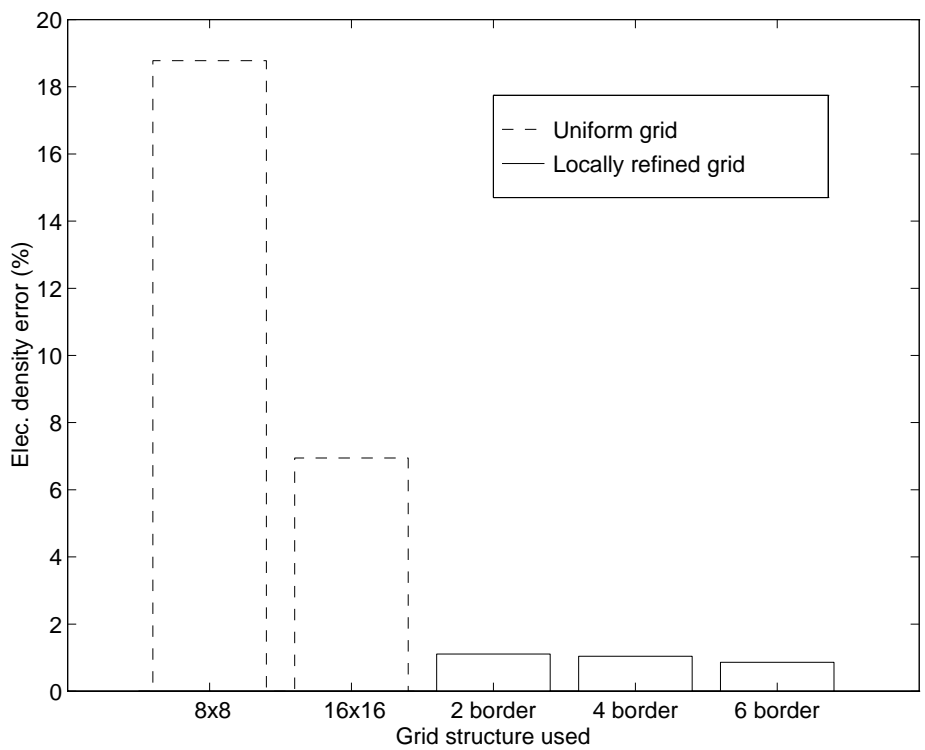

Figure 5.29: Relative error in the electron density at $50 \mu$ s for different refined regions. The labels refer to the number of cells refined near the boundary.

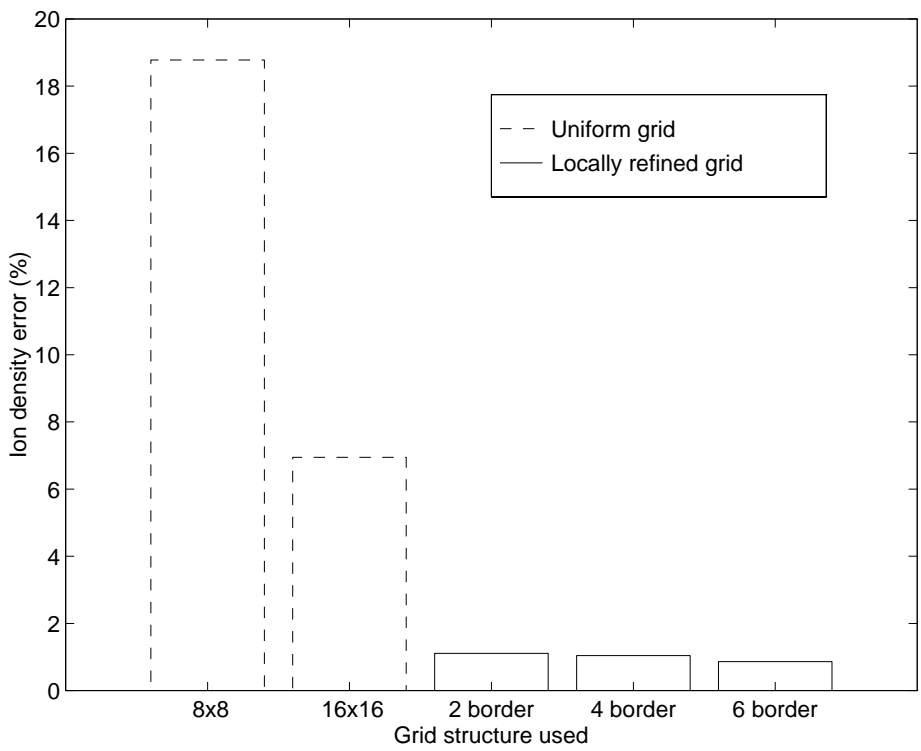

Figure 5.30: Relative error in the ion density at $50 \mu$ s for different refined regions. The labels refer to the number of cells refined near the boundary. 


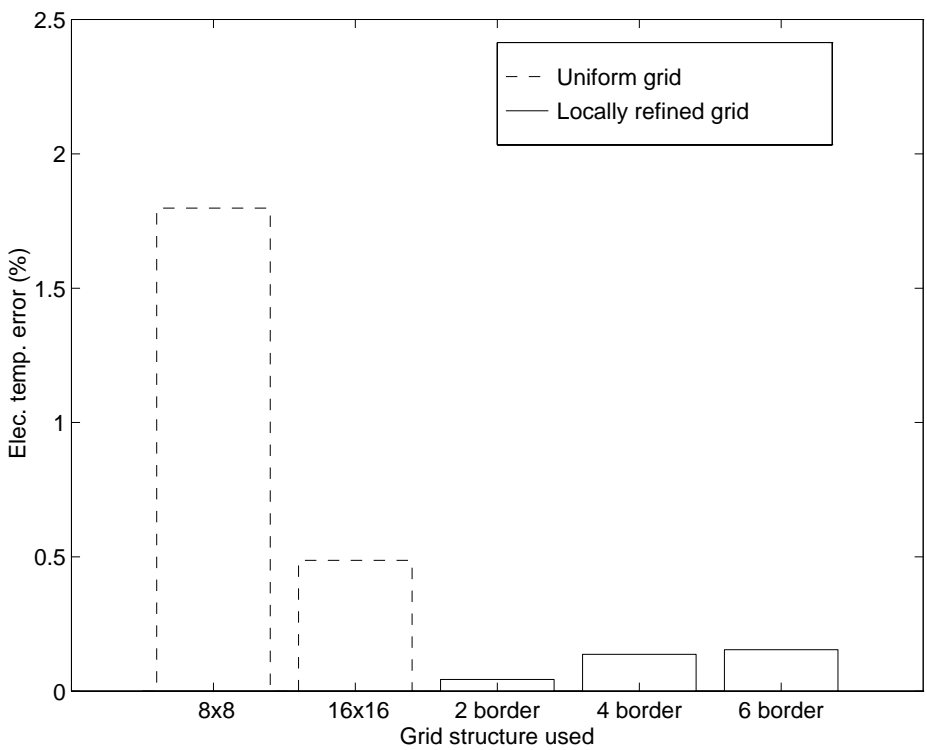

Figure 5.31: Relative error in the electron temperature at $50 \mu \mathrm{s}$ for different refined regions. The labels refer to the number of cells refined near the boundary.

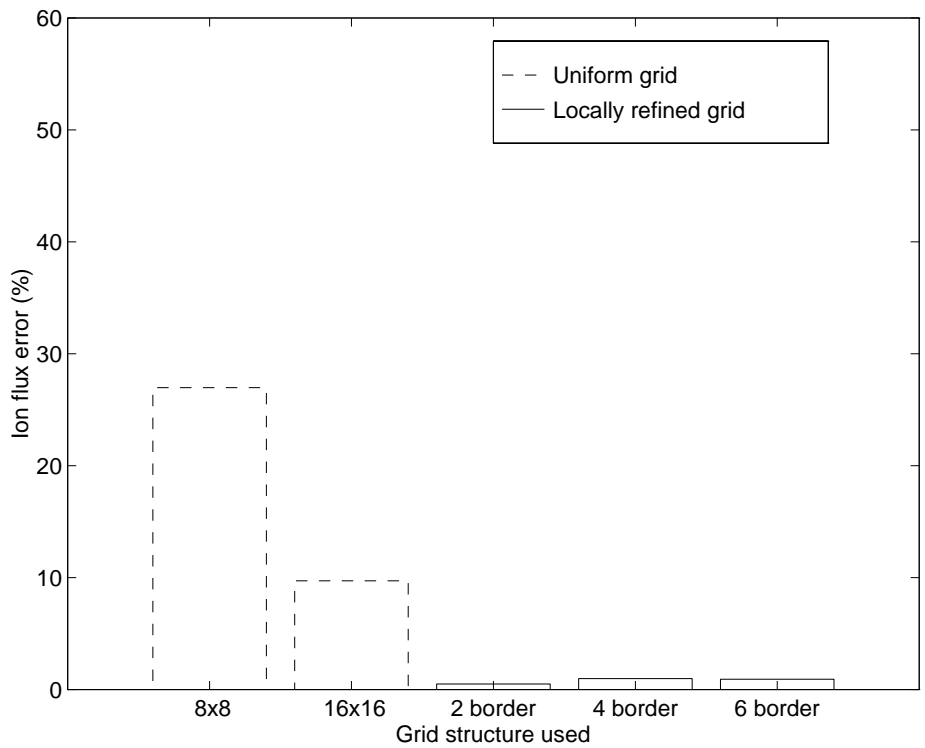

Figure 5.32: Relative error in the ion flux at $50 \mu$ s for different refined regions. The labels refer to the number of cells refined near the boundary. 


\begin{tabular}{|c|c|c|c|c|c|}
\hline Level 0 grid & $16 \times 16$ & $8 \times 8$ & $16 \times 16$ & $16 \times 16$ & $16 \times 16$ \\
\hline $\begin{array}{l}\text { Refinement } \\
\text { ratio level } 1\end{array}$ & NA & NA & 2 & 2 & 2 \\
\hline $\begin{array}{c}\text { Number of } \\
\text { refined edge cells }\end{array}$ & NA & NA & 2 & 4 & 6 \\
\hline $\begin{array}{l}\text { CPU time } \\
\text { (seconds) }\end{array}$ & $2.22 \mathrm{e} 3$ & $4.80 \mathrm{e} 2$ & $3.12 \mathrm{e} 5$ & $3.54 \mathrm{e} 5$ & $3.86 \mathrm{e} 5$ \\
\hline $\begin{array}{l}\text { Potential } \\
\text { (minimum) }\end{array}$ & 15.290 & 54.758 & 0.238 & 0.411 & 0.616 \\
\hline $\begin{array}{l}\text { Net charge } \\
\text { (maximum) }\end{array}$ & 74.484 & 93.153 & 0.484 & 0.722 & 0.634 \\
\hline $\begin{array}{l}\text { Ion density } \\
\text { (minimum) }\end{array}$ & 35.631 & 154.552 & 1.801 & 0.652 & 0.096 \\
\hline $\begin{array}{l}\text { Electron density } \\
\quad(\text { minimum })\end{array}$ & 36.538 & 156.636 & 1.805 & 0.653 & 0.099 \\
\hline $\begin{array}{c}\text { Ion flux } \\
\text { magnitude } \\
\text { (maximum) }\end{array}$ & 11.581 & 38.879 & 0.547 & 0.437 & 0.544 \\
\hline $\begin{array}{c}\text { Electron } \\
\text { temperature } \\
\text { (maximum) }\end{array}$ & 1.943 & 4.826 & 0.486 & 0.618 & 0.614 \\
\hline
\end{tabular}

Table 5.2: Errors relative to high resolution uniform grid calculation for selected quantities at $10 \mu \mathrm{s}$ (values are percent unless otherwise stated).

\subsubsection{Computational Efficiency}

In order for a local refinement strategy to make sense, refined calculations need to show some computational savings, either in CPU time or memory requirements, or both. Figure 5.33 shows the relative CPU times for uniform grid calculations compared to locally refined calculations. The model problem for these timings was an $8 \mathrm{~cm}$ square grid with symmetry boundaries on the top and right side and grounded conductors on the other sides. At each level, the outermost 2 cells along the physical boundary were chosen for refinement. As in previous calculations, 60 mTorr argon with a power density of 0.1 watts $/ \mathrm{cm}^{3}$ was used. One of these refined grids (corresponding to 4 levels refined by a factor of 2) is shown in Figure 5.35.

Results are shown for refinement ratios of 2 and 4 . In each case calculations were performed with a refined grid and a uniform grid. The uniform grid cell size corresponded to the finest cells in the refined grid. Each pair of calculations was run to the same simulation time and the ratio of 


\begin{tabular}{|c||l|r|}
\hline Factor limiting timestep & Timestep & $\begin{array}{c}\text { Number of steps } \\
\text { to steady state }\end{array}$ \\
\hline \hline $\begin{array}{c}\text { Ion Courant condition } \\
\text { (quasineutral region) }\end{array}$ & $4.89 \mathrm{e}-6$ & 102 \\
\hline $\begin{array}{c}\text { Ion Courant condition } \\
\text { (sheath region) }\end{array}$ & $1.77 \mathrm{e}-7$ & 2,825 \\
\hline $\begin{array}{c}\text { Electron drift velocity Courant condition } \\
\text { (quasineutral region) }\end{array}$ & $1.64 \mathrm{e}-8$ & 30,487 \\
\hline $\begin{array}{c}\text { Electron drift velocity Courant condition } \\
\text { (sheath region) }\end{array}$ & $2.03 \mathrm{e}-10$ & $2,463,054$ \\
\hline Dielectric relaxation time & $2.50 \mathrm{e}-15$ & $200,000,000,000$ \\
\hline
\end{tabular}

Table 5.3: Comparison of computational complexity for different limiting timesteps.

CPU times was calculated. Because of prohibitive runtimes for the higher resolution cases (uniform or locally refined), the final time for each pair of calculations was the same but different pairs used different final times. Our experience has been that the CPU time per timestep does not vary much during a calculation, so these timings should be representative.

We have mentioned the dielectric relaxation time and how hard these problems are computationally. Perhaps it is useful to illustrate this by considering the different timescales and timesteps based on different physical phenomena. Table 5.3 shows the steady state timesteps used for a sample $64 \times 64$ cell calculation as well as the number of such steps required to get to $500 \mu s$ (steady state). As discussed before, the limiting timestep in our current algorithm is based on the electron drift velocity in the sheath region. The consequence of this is that steady state calculations are very difficult (though with the dielectric relaxation limit, they would be impossible). If we could base the algorithm timestep on the ion motion only, we would be in much better shape. This is the focus of current research.

That being said, we have still gained alot using local refinement. Figure 5.33 shows that speedup is a strong function of refinement ratio. For the model problem, 4 refined levels were required to obtain an improvement over the single fine grid case using a refinement ratio of 2 . When a refinement ratio of 4 was used, only 2 refined levels were required to get a significant speedup. 
These trends depend on a number of factors. Chief among these are the time spent performing composite synchronizations and the fraction of the domain covered by fine cells. For this model problem, the number of cells at the fine levels was rather large compared to the coarse levels. This is required if one wants to refine along an entire edge. If, for example, a corner was recursively refined we would expect an ever larger speedup using the locally refined grid.

The dependence of time spent performing composite synchronizations on the grid hierarchy is hard to quantify, but is typically about $20-30 \%$ of the total simulation time. We do know that the number of composite solves grows quickly with the number of levels. If $m$ refined levels are used and each is refined by a factor of $n$ in time and space, this will require $n^{m-1}$ composite solves per coarse timestep. This fact and the speedup results shown in Figure 5.33 both suggest the benefit of higher refinement ratios.

Decreased runtime is not the only benefit of locally refined grids. Figure 5.34 shows the relative reduction in memory requirements for the locally refined calculation. The memory requirements are much less severe for refined grids, provided the finest levels do not make up a large fraction of the domain. At some level of refinement, it is no longer feasible to compare highly refined grids to uniform equivalent grids because the latter cannot fit within physical memory. This fact limited the number of refined levels we could use in these comparisons.

The finest of the calculations shown in Figure 5.33 cannot be run to steady state in a reasonable amount of time. Even with the speedup shown with local refinement, very high resolution calculations are still very expensive. Steady state solutions may be more easily obtained by gradually refining the grid in time. This approach is investigated in a later section.

\subsubsection{Multilevel Timestep Selection}

One of the most compelling reasons for adopting the locally refined grid approach is to limit the number of cells which require small timesteps and to use larger timesteps elsewhere. The Courant $\left(\Delta t \leq \Delta x / v_{d r i f t}\right)$ condition typically forces the use of the smallest timesteps on the finest 


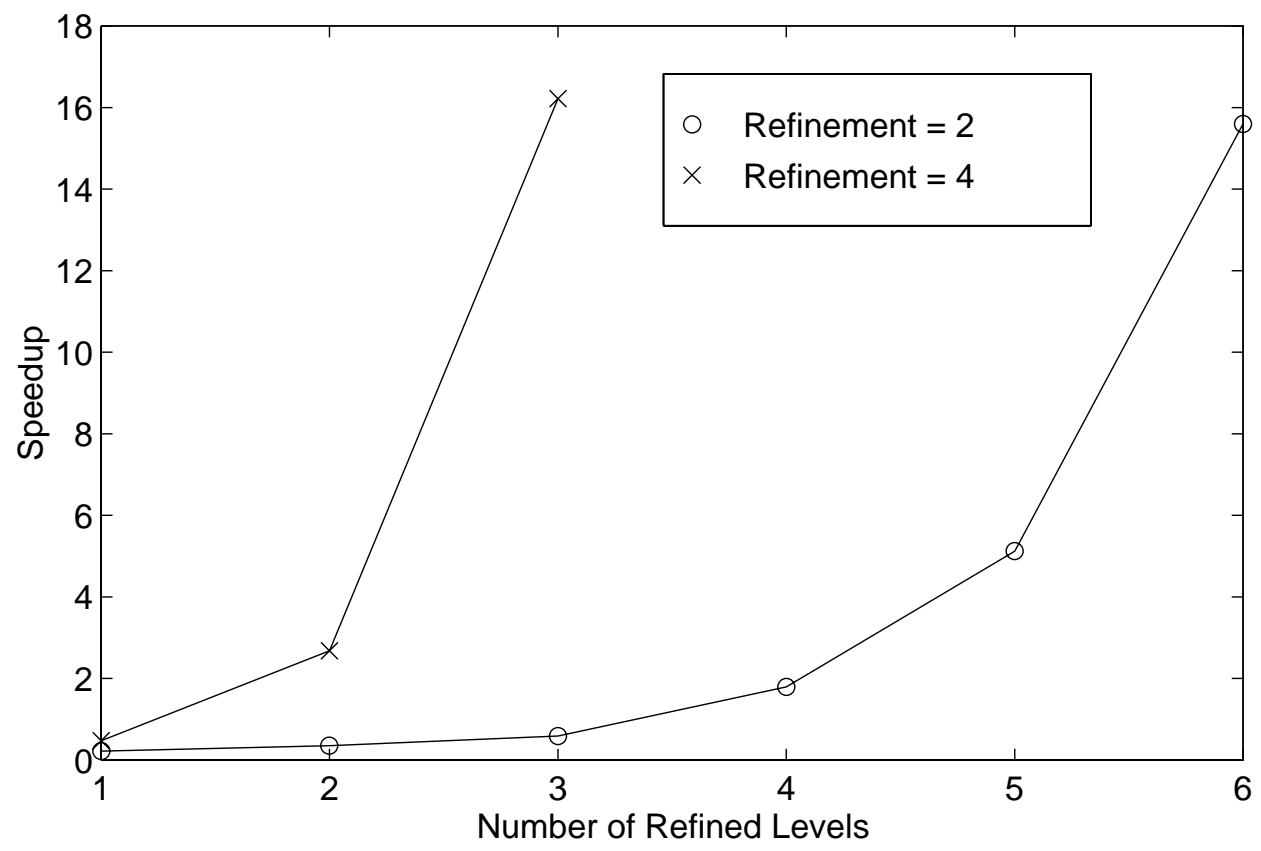

Figure 5.33: Ratio of CPU times for uniform and locally refined grid calculations.

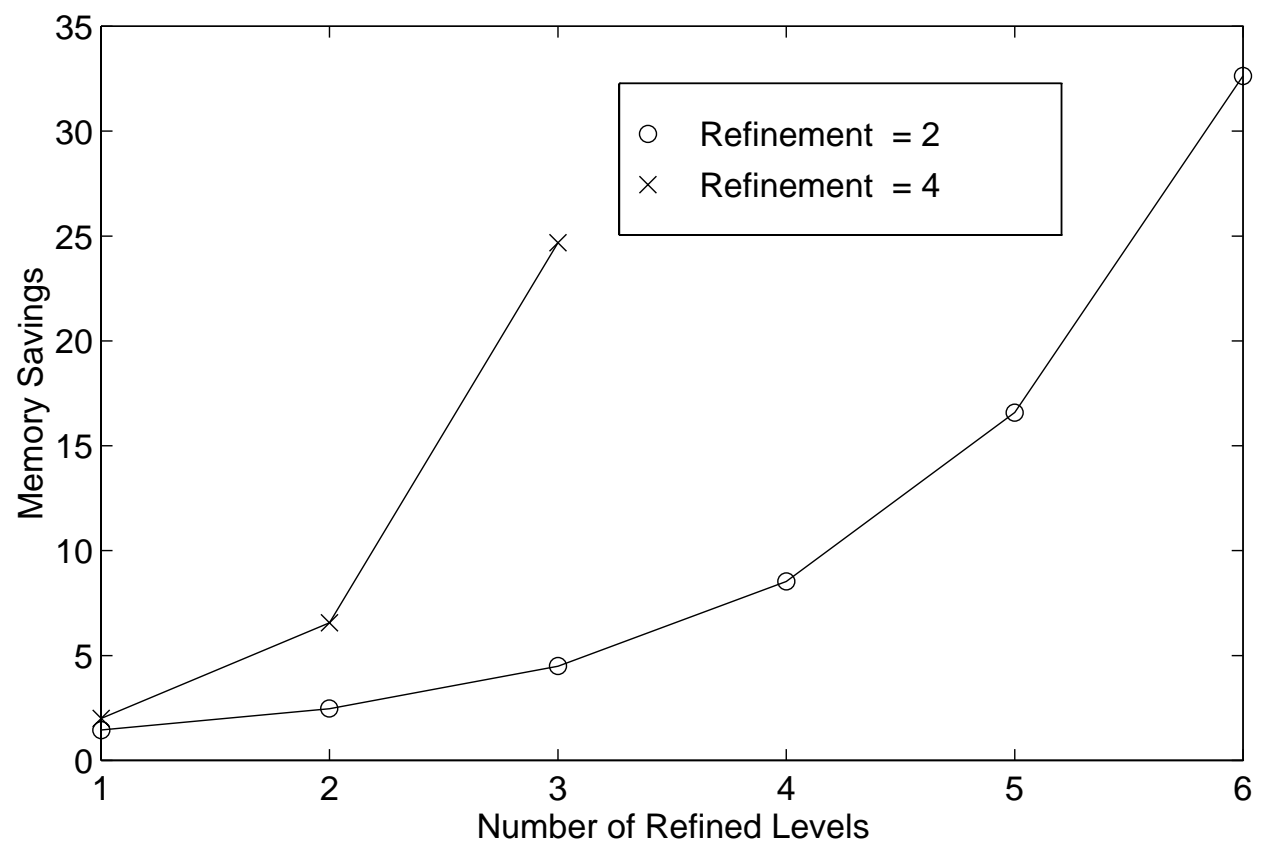

Figure 5.34: Ratio of memory requirements for uniform and locally refined grid calculations. 


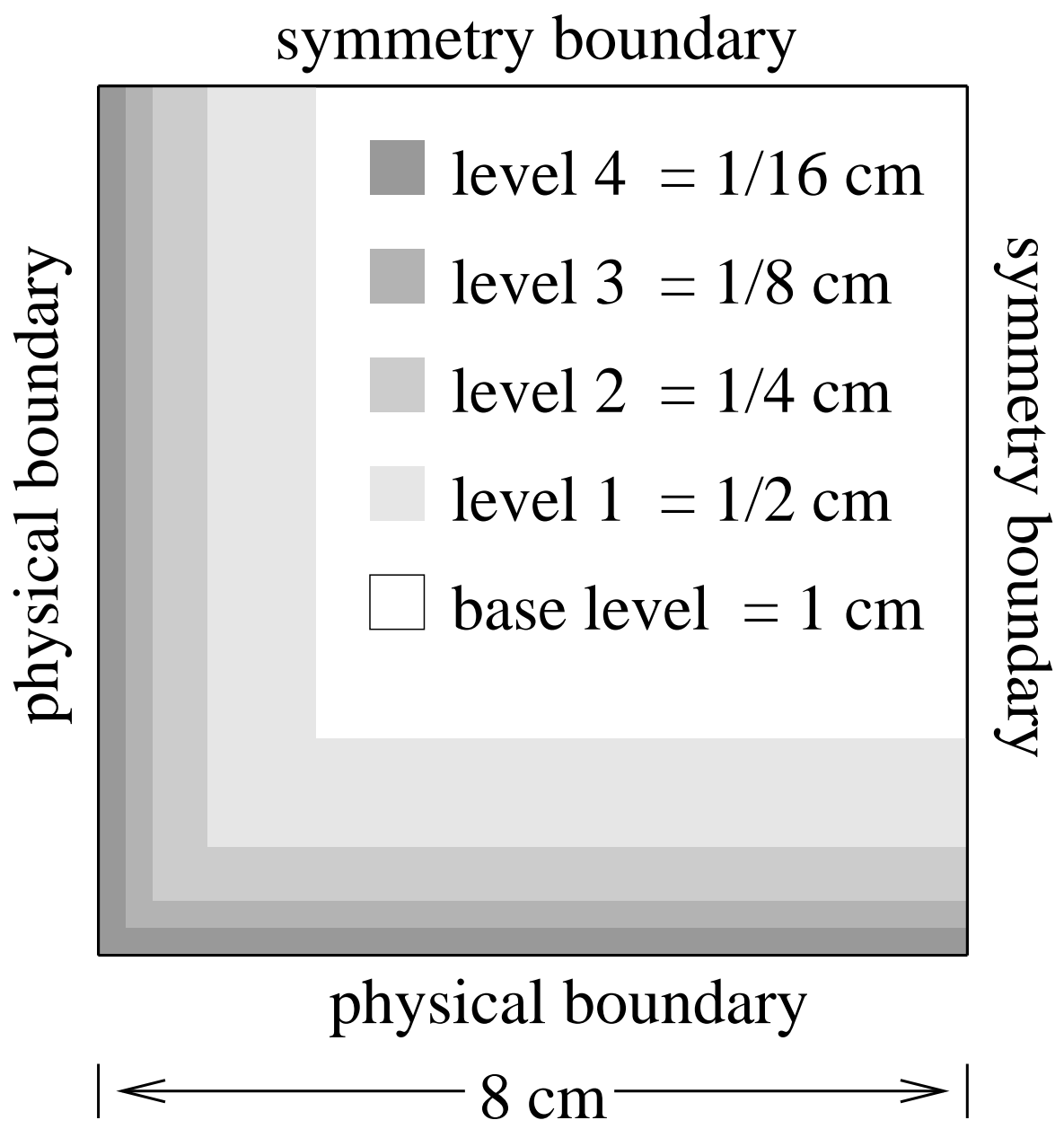

Figure 5.35: Sample grid used to calculate time and storage savings shown in Figure 5.33. This grid corresponds to the case with refinement ratios of 2 and 4 refined levels. 
grids. For our application, the limiting timesteps do not usually vary in direct proportion to the cell size, however. This is because of the peak drift velocities which vary from level to level and cell to cell. In this application, the peak drift velocities usually occur near the physical boundary and these regions tend to have the smallest cells. These factors combine to give a maximum level timestep, $t_{\ell}$, which varies not by $\Delta x_{\ell}$ but by some other factor. In some cases this factor turns out to be closer to $\Delta x_{\ell}^{2}$. This suggests that one might want to use level timesteps which vary super-linearly with cell size. We term this approach accelerated subcycling. There may be other factors determining the relationships between timesteps as well. For example, we might decide to use the same timestep on all levels. This has the advantage of eliminating many of the approximations made in Chapter 4 for the composite synchronizations. This approach might be best from an accuracy point of view, albeit at some computational expense. We refer to this timestep selection as the no subcycling approach. Intermediate to these is the traditional hyperbolic AMR method of standard subcycling, in which we use level timesteps which vary in inverse proportion to the spatial refinement ratios. Figure 5.39 shows these different strategies schematically.

Without loss of generality, assume that the peak level drift velocities, $v_{\ell}$, are ordered $v_{0} \leq v_{1} \leq v_{2} \ldots \leq v_{M}$, where $M$ is the maximum level number. This assumption does not greatly effect the timestep selection but simplifies the discussion. If we define $n_{\ell}^{\text {cycle }}$ as the number of level $\ell$ timesteps needed to equal one level $\ell-1$ timestep, we can write the following relation for the timestep used for level $\ell$

$$
\Delta t_{\ell}=\left(\prod_{i=\ell+1}^{M} n_{i}^{c y c l e}\right) \Delta t_{M} .
$$

The three multilevel timestep selection procedures we will consider can then be described by the values used for $n_{i}^{\text {cycle }}$ as follows 


\begin{tabular}{|c|c|}
\hline $\begin{array}{c}\text { Fine steps } \\
\text { per } \\
\text { coarse step }\end{array}$ & $\begin{array}{c}\text { CPU time } \\
\text { (seconds) }\end{array}$ \\
\hline 1 & $2.45 \mathrm{e} 5$ \\
4 & $1.35 \mathrm{e} 5$ \\
16 & $9.61 \mathrm{e} 4$ \\
32 & $9.08 \mathrm{e} 4$ \\
\hline
\end{tabular}

Table 5.4: Timings for different subcycle strategies.

$$
n_{i}^{\text {cycle }}=\left\{\begin{array}{lll}
1 & ; \text { no subcycling } \\
n_{i}^{\text {ref }} & ; \text { standard subcycling } \\
\beta_{i} n_{i}^{\text {ref }} & ; \text { accelerated subcycling }
\end{array}\right.
$$

where $n_{i}^{r e f}$ is the spatial refinement ratio and $\beta_{i}$ is an acceleration factor (restricted so that $\beta_{i} n_{i}^{r e f}$ is a positive integer).

To help understand the effect of subcycling on accuracy and efficiency, four calculations were performed and compared. The first used no subcycling, the second used standard subcycling, the third used accelerated subcycling with $\beta=4$ and the fourth used accelerated subcycling with $\beta=8$ (accept for a few microseconds at the start in which $\beta=4$ was required for stability). Each was a two level problem in which the 2 boundary cells were refined by a factor of 4 . The level 0 grid was $8 \times 8$. Each case was run to $100 \mu s$. These locally refined calculations were then compared to an equivalent uniform grid calculation. The norm, (5.8), was calculated for various quantities of interest at $10 \mu s, 50 \mu s$ and $100 \mu s$, and the results are shown in Figures 5.36, 5.37 and 5.38. Timings are given in Table 5.4.

It is difficult to draw conclusions from a single study, but these calculations give us some indication of the effects due to the multilevel timestep selection. Consider the $100 \mu s$ case. The highly accelerated case $(\beta=8)$ is highly inaccurate for the densities and ion flux. Although it is the fastest, it is less than 10 percent faster than the $\beta=4$ case. This would seem to imply that the extra acceleration is not worth the accuracy penalty. On the other end of the scale, the no subcycle 

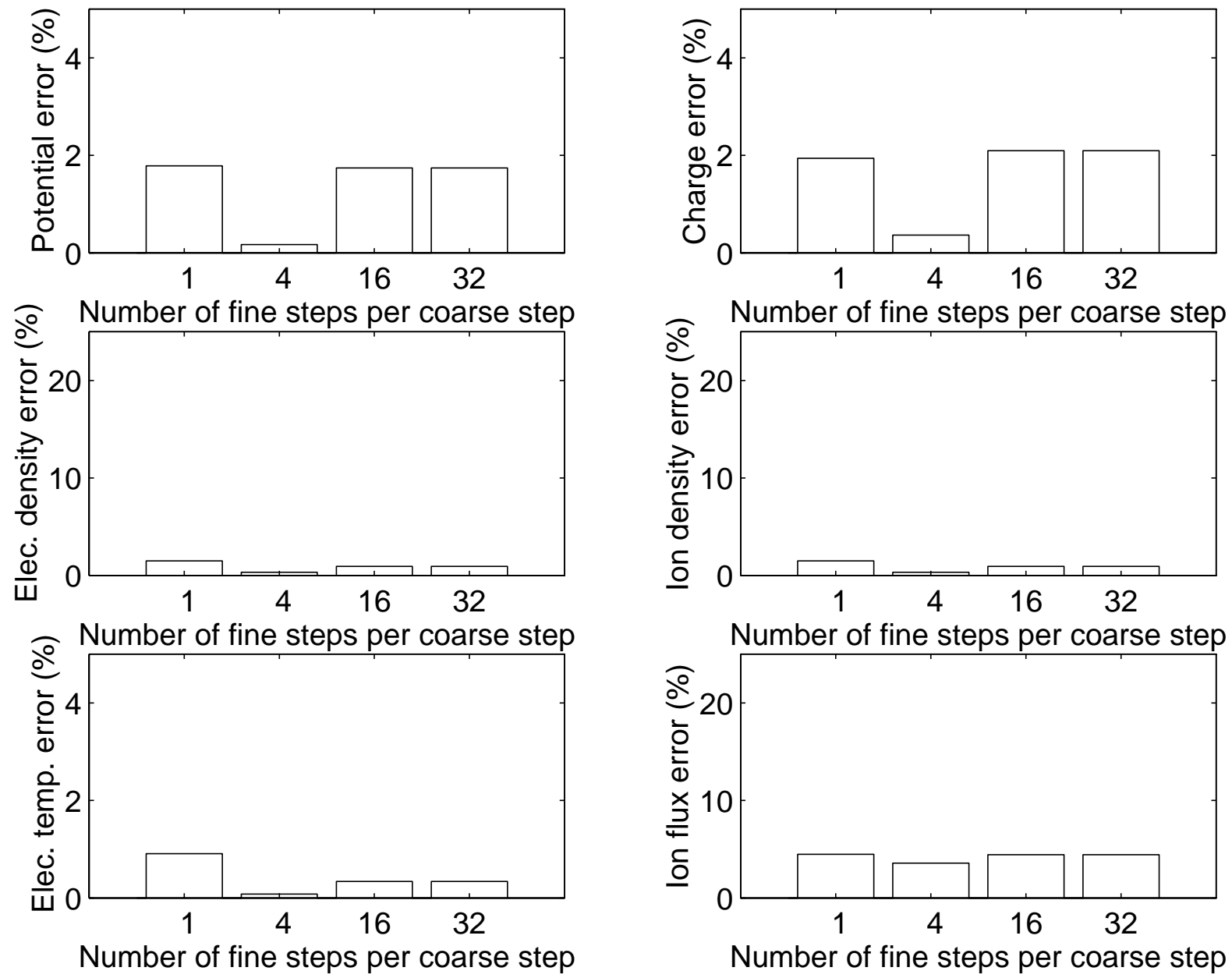

Figure 5.36: Errors for subcycle study at $10 \mu s$. 

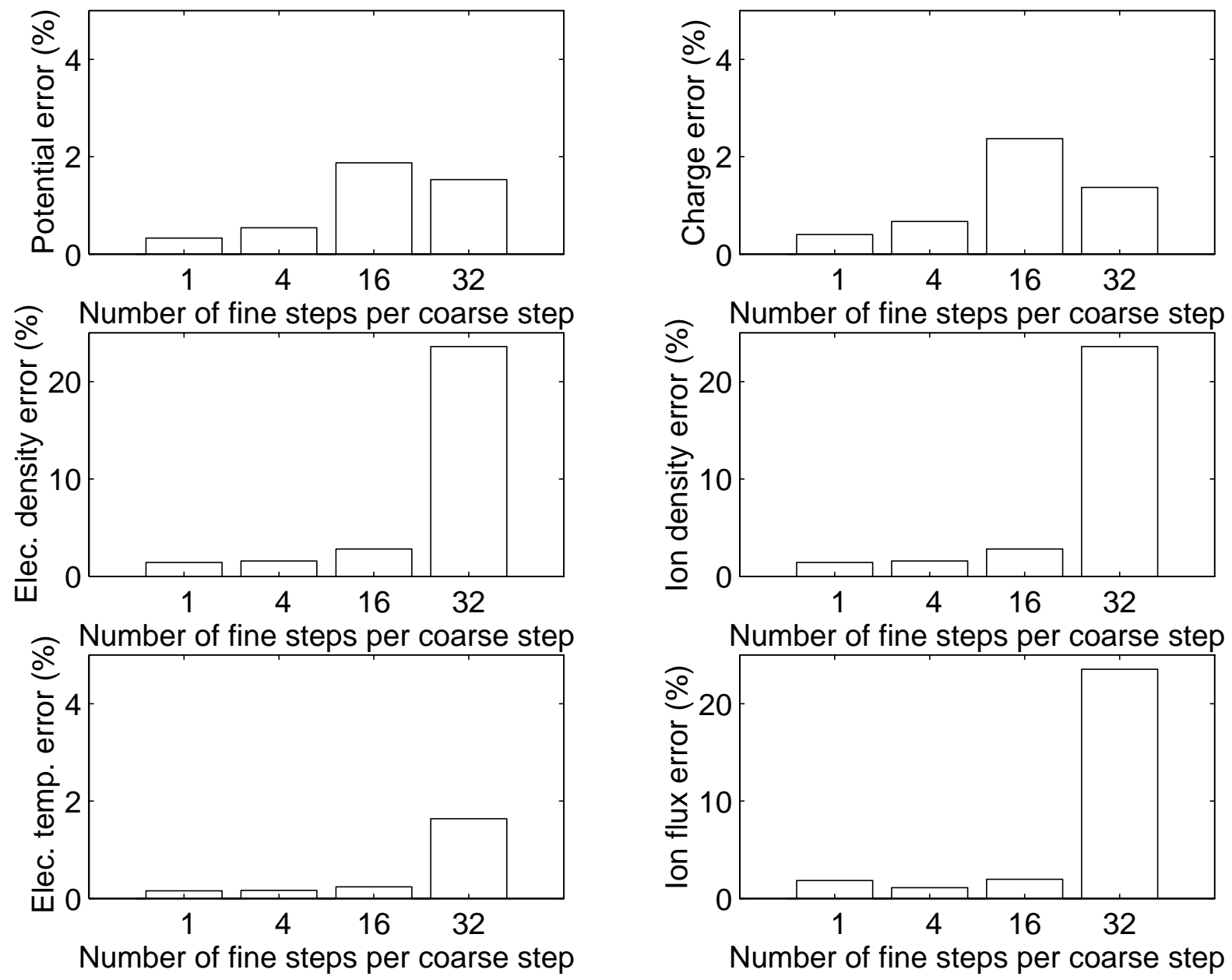

Figure 5.37: Errors for subcycle study at $50 \mu s$. 

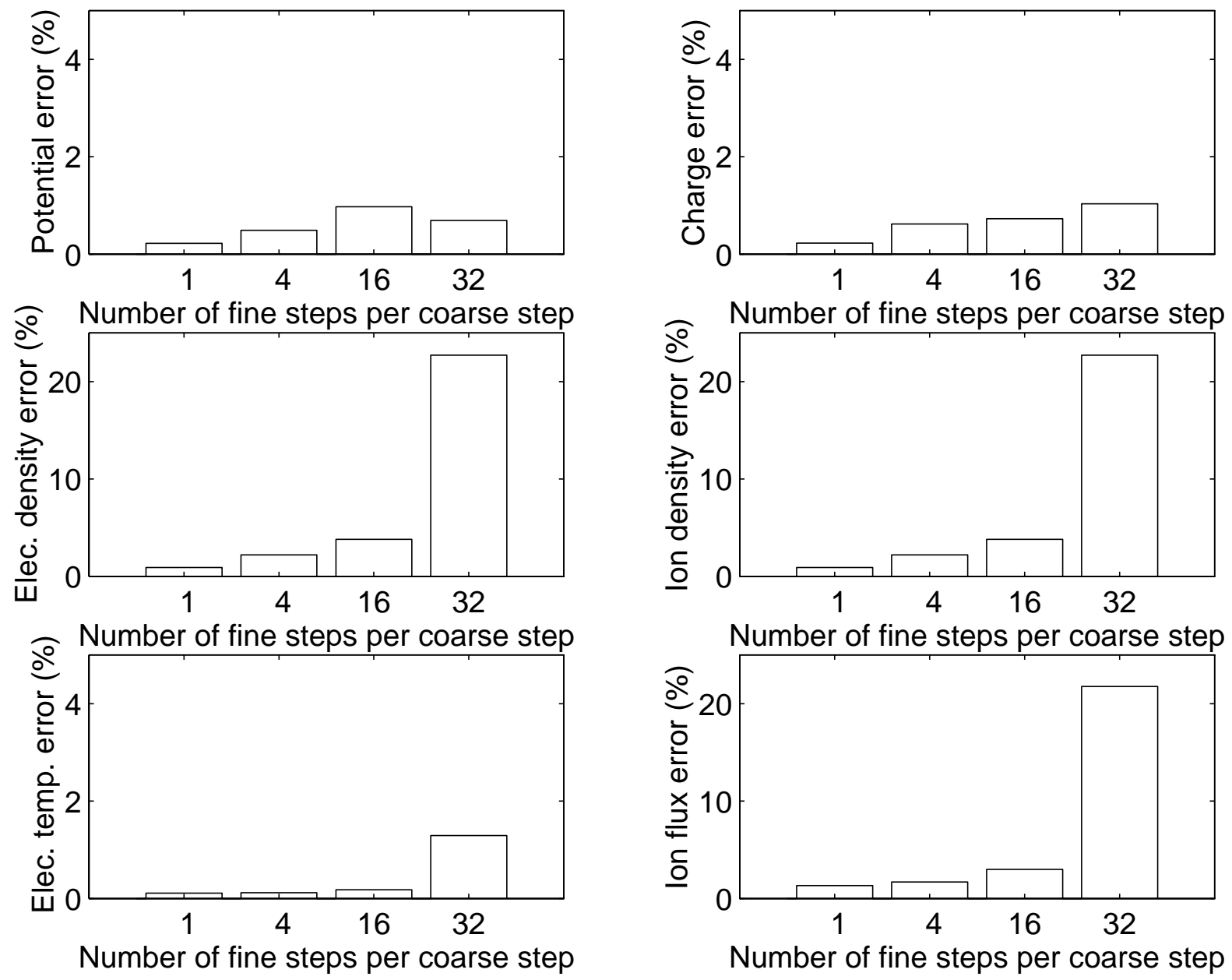

Figure 5.38: Errors for subcycle study at $100 \mu s$. 
case was the most accurate (as expected). It was nearly twice as expensive as the standard subcycle case, however. Though less accurate, the standard subcycle case still was within a few percent of the uniform grid case for all variables. Depending on the user, this may be an acceptable level of accuracy considering the time savings. The accelerated case with $\beta=4$ was also very accurate (within 4 percent for all variables) and gave an additional time savings over the standard subcycle case of almost 50\%. Again, the accuracy penalty may be worth the time savings.

In light of the approximations we make and the importance we have placed on maintaining the Poisson equation constraint, these results are not surprising. Even in the most aggressive subcycling case, the potential and charge errors are small. This seems to say that our diligence in accounting for charge imbalance in the composite synchronizations has paid off. The infrequent communication between levels caused large errors in the densities and ion flux, however. This aggressive approach, therefore, does not seem to limit the stability (which is primarily limited by the ability to maintain the Poisson constraint), but does hurt the accuracy. Further study as well as consideration of the relative expense of the level advances versus synchronization steps is needed.

The trends are not so clear for the solutions at $10 \mu s$ and $50 \mu s$. It is interesting to note the anomalously low error for the standard subcycle case at $10 \mu s$. For certain discretizations of the advection-diffusion equation, the use of a timestep near the Courant limit results in cancellation of terms in the local truncation error [33]. Perhaps that is what is going on here, but it is only speculation.

\subsubsection{Adaptivity}

As is the case for timestep selection, the choice of where and when to add or delete grids impacts the accuracy and efficiency of the method. Transient simulations evolved to steady state using highly refined grids are currently computationally intractable. We therefore wish to investigate the advantage gained by adding resolution as the solution evolves. The thought is that the time to achieve a steady state will be reduced if the starting conditions are already near the correct solution. 

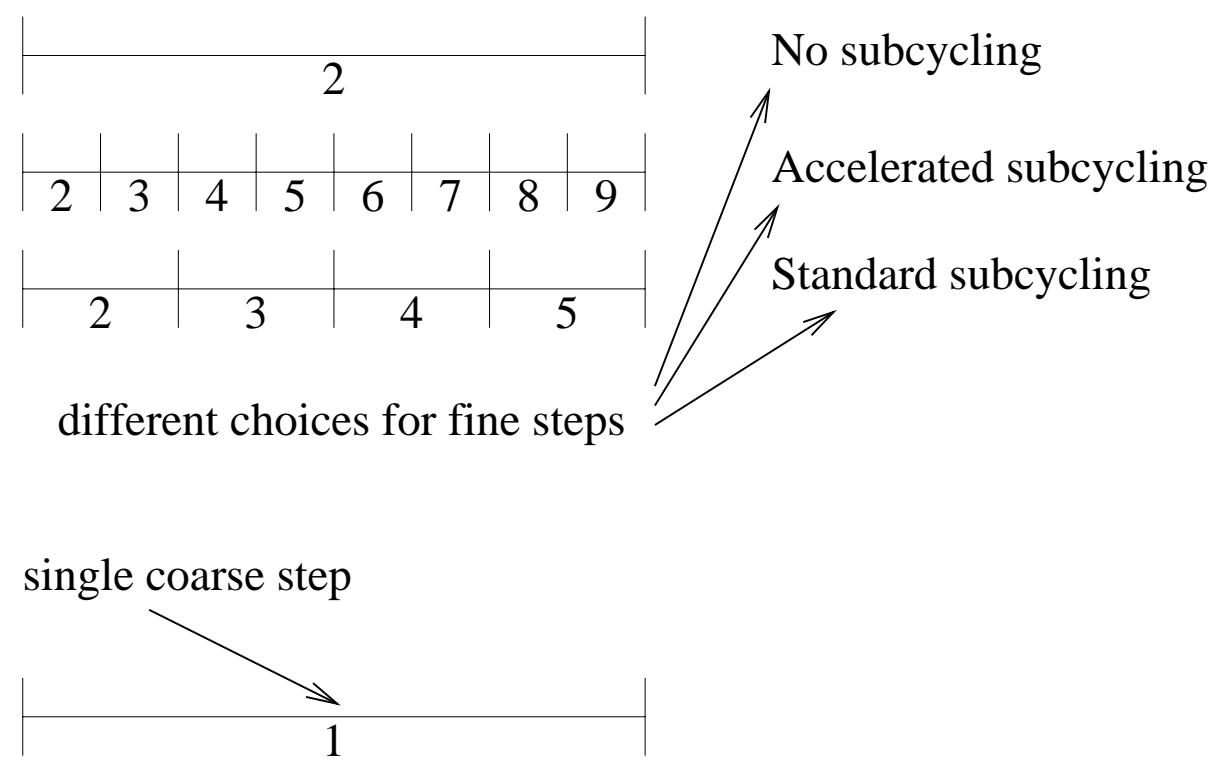

Figure 5.39: Order of grid advances to evolve a two level problem with a refinement ratio of 4 to a new, common time. The upper intervals represent the fine grid and lower interval represents the coarse grid.

We refer to this gradual refinement as the gridding schedule. In addition to choosing when to add grids, we must decide where to add them.

\section{Criteria for Refinement}

Traditionally, there are three ways in which cells are chosen for refinement. One method is to use Richardson extrapolation. The model is evolved with a certain spatial resolution and then reevolved with a coarser resolution. If the solutions differ more than a specified tolerance in some region, then it is determined that the region needs refinement. We have not implemented this procedure because of its complexity and expense. A second method is to base refinement on physical parameters of the solution. For example, one might want to refine in regions where large gradients in certain key variables are present. For our application, in which sheath and pre-sheath behavior is important, regions with significant charge are candidates for refinement. Finally, grids can be placed in an ad-hoc fashion. In this case, the user simply decides apriori where he wants the grids based on physical insight. Due to the importance of the boundary regions, we have generally been content 
to use fixed grids which are chosen apriori to give high resolution near the physical boundary. This is the basis for the grid selection used in calculations in subsequent chapters.

Another reason for using fixed grids is interpolation error. In changing from one set of grids to another, some interpolation scheme must be used to initialize the new grid structure. This necessarily introduces some error into the solution. In the absence of solution features which move in time (such as shocks in fluids or cracks in structural problems, for example) the repeated introduction of this error seems too great a price to pay to frequently regrid.

\section{Adaptivity in Time and Acceleration to Steady State}

The locally refined calculations presented in Chapters 6 and 7 are all steady state solutions. They were obtained by starting with a uniform coarse grid, evolving the solution to steady state, and then refining part of the grid. The new, refined grid needs to be initialized and this is done through conservative bilinear interpolation. This new solution is not necessarily in steady state, however. Consequently this new grid must be evolved to steady state as well. In the case of the hydrogen sheath simulation, this refinement process was repeated and a third level was added and brought to steady state.

Presumably the steady state solution to a coarse problem will provide an initial condition on the refined grid which is better than our standard neutral, constant density, zero flux initial condition. One would imagine that the time to steady state would be greatly reduced with such an initial condition. The aforementioned interpolation error admits some error which must die away before the new solution is converged. In order to quantify this tradeoff, the runtimes obtained for the refined calculations in Chapters 6 and 7 were compared to the time it would have taken if we had run the most refined grid from the start. The estimates for run times using the refined grid throughout the calculation were obtained by calculating the ratio of CPU time to simulation time for the finest grid structure and assuming the time it would take to steady state was the same as for the coarse, uniform single-level grid. These timings are shown in Table 5.5. 
For most of the cases in Table 5.5, the speedup due to refinement in time was from 3 to 3.5 . The hydrogen sheath case was not as good, resulting in a speedup of only about 1.5. In this case, the two level steady state calculation was simply not a good approximation to the three level case. Perhaps the difference is due to the change in resolution from a grid spacing which was larger than the sheath width to one which was smaller. Alternatively, it could be that this speedup diminishes as we go to higher and higher resolutions in general. This has not been our experience, however. In any event, this method demonstrates computational savings though in general the amount of savings in unclear. 


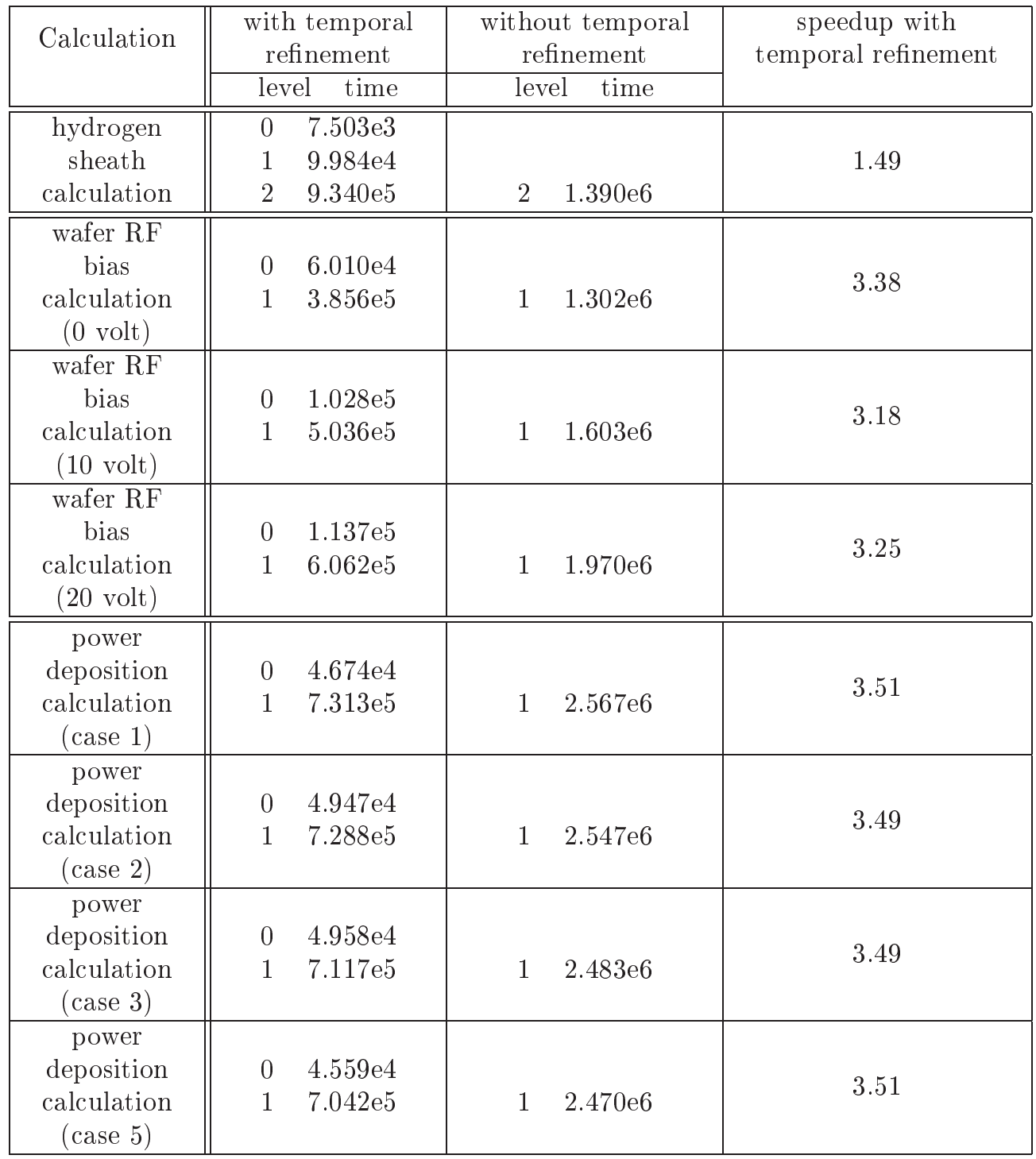

Table 5.5: Comparison of timings for applications calculations using refinement in time with estimates for equivalent static grid calculations. Times are in seconds and are cumulative. 


\section{Chapter 6}

\section{Applications I : Sheath Scale}

\section{Hydrogen Plasma Simulation}

One of the primary motivations for using locally refined grids is to allow for the simulation of features with disparate length scales. In this chapter, we illustrate this by modeling a hydrogen plasma with sheath-scale resolution in a domain that is a few hundred times larger. Specifically, we take advantage of symmetry and model an $8 \mathrm{~cm}$ region with a $4 \mathrm{~cm}$ long volume and a symmetry boundary condition. In addition to the symmetry boundary in the x-direction, we enforce symmetry in the transverse direction. This forces the variation in the solution to one direction, making it a one-dimensional problem. We employ two refined levels, each with a refinement ratio of 4 . Each refined level covers the exterior 1/4 of the next coarser level. This grid structure is shown in Figure 6.1. With these refinements, the finest cells are $156 \mu \mathrm{m}$, which turns out to be approximately the Debye length. At this resolution, we have a grid spacing that is smaller than the expected plasma 


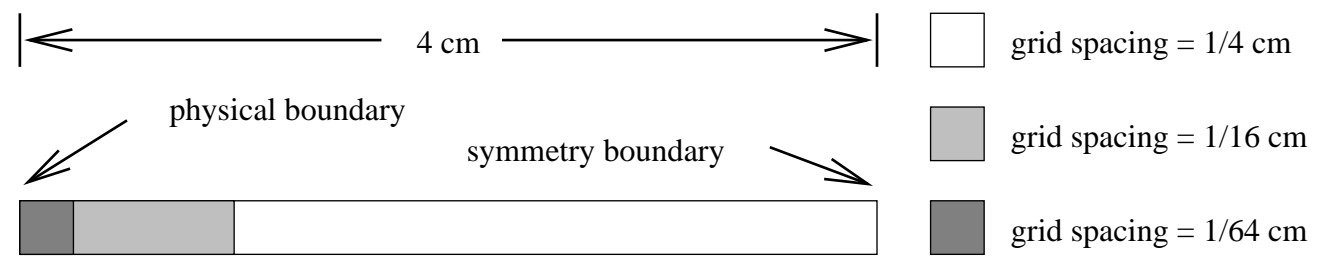

Figure 6.1: Grid structure for hydrogen sheath calculation.

sheath width.

\subsection{Ingold Model}

Ingold's [37] sheath model is a good one to compare to our code. It uses a fluid description of the plasma and does not make a number of approximations which are common in plasma sheath models. For example, it does not assume a Boltzmann distribution for the sheath electrons, nor does it ignore ionization in the sheath.

The analytic model we compare to our numerical results is actually slightly different from Ingold's. In Ingold's work, the ionization source term in the continuity equation is not included in all equations (either due to an error or the assumption that it is small). In this work it is consistently included. Secondly, rather than solve a set of equations for initial conditions, we obtain the initial conditions directly from the code results.

Though similiar, our model differs from that used in [37] in a number of important ways. Most significantly, we are solving for the electron temperature while the Ingold model assumes a constant temperature. This is important in that the ionization and collision frequencies depend on the electron temperature. Also, we are making the drift-diffusion approximation for the electron momentum while the Ingold model uses the full momentum equation. If our drift-diffusion approximation is a good one, this should not be a big effect.

The description of the plasma includes the continuity, momentum conservation and Poisson equations 


$$
\begin{aligned}
\frac{\partial,}{\partial x} & =\nu_{i z} n_{e} \\
m_{e} \frac{\partial}{\partial x}\left(\frac{2}{n_{e}}\right) & =-k T_{e} \frac{\partial n_{e}}{\partial x}-e n_{e} E-m_{e} \nu_{e n},+m_{e} \nu_{i z}, \\
m_{i} \frac{\partial}{\partial x}\left(\frac{{ }^{2}}{n_{i}}\right) & =-k T_{i} \frac{\partial n_{i}}{\partial x}+e n_{i} E-m_{i} \nu_{i n},+m_{i} \nu_{i z}, \frac{n_{e}}{n_{i}} \\
\frac{\partial E}{\partial x} & =\frac{e}{\epsilon_{0}}\left(n_{i}-n_{e}\right)
\end{aligned}
$$

where $n_{e}, n_{i}$, , and $E$ are the electron density, ion density, flux, and electric field, respectively. Note that in the steady state the electron and ion fluxes are equal, so only one flux variable is needed. The temperatures $T_{e}$ and $T_{i}$ are assumed constant, as are the collision and ionization frequencies.

Following [37], we introduce dimensionless variables.

$$
\begin{aligned}
x & =\alpha \xi \\
n_{e} & =a_{1} N \\
n_{i} & =a_{1} P \\
, & =a_{2} G \\
E & =a_{3} H
\end{aligned}
$$

With the constants defined as

$$
\begin{aligned}
a_{1} & \equiv \frac{m_{i} \epsilon_{0} \nu_{i n} \nu_{i z}}{e^{2}} \\
a_{2} & \equiv a_{1} \sqrt{\frac{n u_{i z} k T_{e}}{m_{e} \nu_{e n}}} \\
a_{3} & \equiv \frac{m_{i} \nu_{i n}}{e} \sqrt{\frac{n u_{i z} k T_{e}}{m_{e} \nu_{e n}}} \\
\alpha & \equiv \sqrt{\frac{k T_{e}}{m_{e} \nu_{i z} \nu_{e n}}}
\end{aligned}
$$

Make the further definitions 


$$
\begin{aligned}
\rho & \equiv \frac{T_{i}}{T_{e}} \\
\sigma & \equiv \frac{m_{i} \nu_{i n}}{m_{e} \nu_{e n}} \\
\delta & \equiv \frac{m_{e}}{m_{i}} \\
\tau_{+} & \equiv \frac{\nu_{i z}}{\nu_{i n}} \\
\tau_{-} & \equiv \frac{\nu_{i z}}{\nu_{e n}}
\end{aligned}
$$

With these variables, the system is transformed into the following

$$
\begin{aligned}
\frac{\partial N}{\partial \xi} & =-\frac{\left(1+\tau_{+}\right) G+\sigma N H}{1-\tau_{-} G^{2} / N^{2}} \\
\frac{\partial P}{\partial \xi} & =\frac{\sigma\left(H P-G\left[1+\tau_{+} N / P\right]\right)}{\rho-\left(\tau_{-} / \delta\right)\left(G^{2}\right) /\left(P^{2}\right)} \\
\frac{\partial G}{\partial \xi} & =N \\
\frac{\partial H}{\partial \xi} & =P-N
\end{aligned}
$$

We now have a system of ordinary differential equations to be solved. Equations (6.5) and (6.6) have possible singularities, but it turns out that only the denominator in (6.6) poses any problem. This term is singular where the ion velocity is a factor of $\sqrt{T_{i} / T_{e}}$ smaller than the Bohm velocity. So it occurs well into the quasineutral plasma, not in the sheath region.

\subsection{Comparison with APM}

In order to verify that our algorithm properly models the plasma behavior in the sheath, within the limits of our fluid model, we compared the APM results to the sheath model of Ingold [37]. For the comparison, we took APM calculated values for ion and electron density, flux and electric field at a point outside of the sheath region and used this data as an initial condition for the Ingold model. The model was then integrated to the bounding wall using CVODE $[15,16]$. 


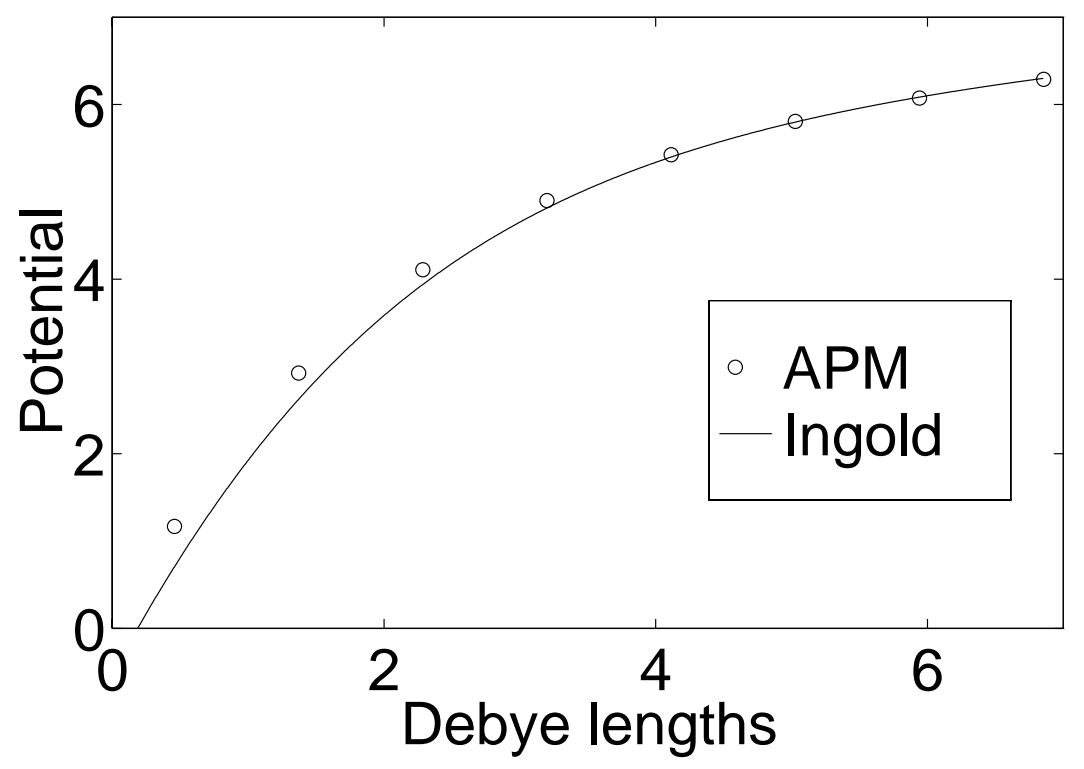

Figure 6.2: Comparison of APM and Ingold sheath model for potential.

These results were then compared with the APM results in the sheath region and are shown in Figures 6.2 through 6.4. The frequency values for electron impact ionization, electron-neutral and ion-neutral elastic collisions where $1.3710^{5} \mathrm{~s}^{-1}, 8.6110^{7} \mathrm{~s}^{-1}$, and $2.3110^{6} \mathrm{~s}^{-1}$, respectively [56].

The agreement is excellent. The small variations may be attributable to the omission of electron inertia in APM or different treatments for the boundary conditions. Discretization error is the most likely cause. In the region shown in Figures 6.2 through 6.4, we have less than ten grid points in the APM calculation while the Ingold calculation has many hundreds. The assumption of constant electron temperature and constant collisional parameters in the Ingold model was found to have no effect on the comparison. This was determined by varying the temperatures and collisional parameters in the Ingold model within the range used in the APM calculation. 


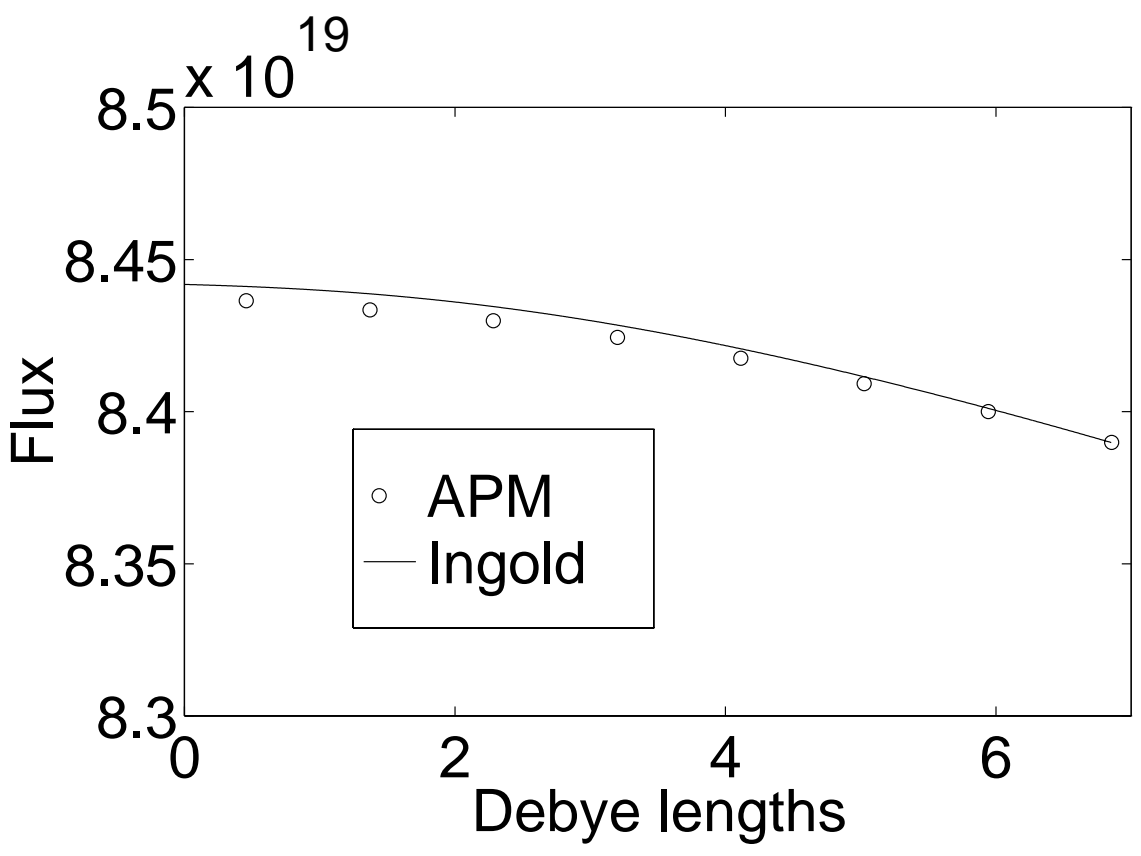

Figure 6.3: Comparison of APM and Ingold sheath model for flux. 


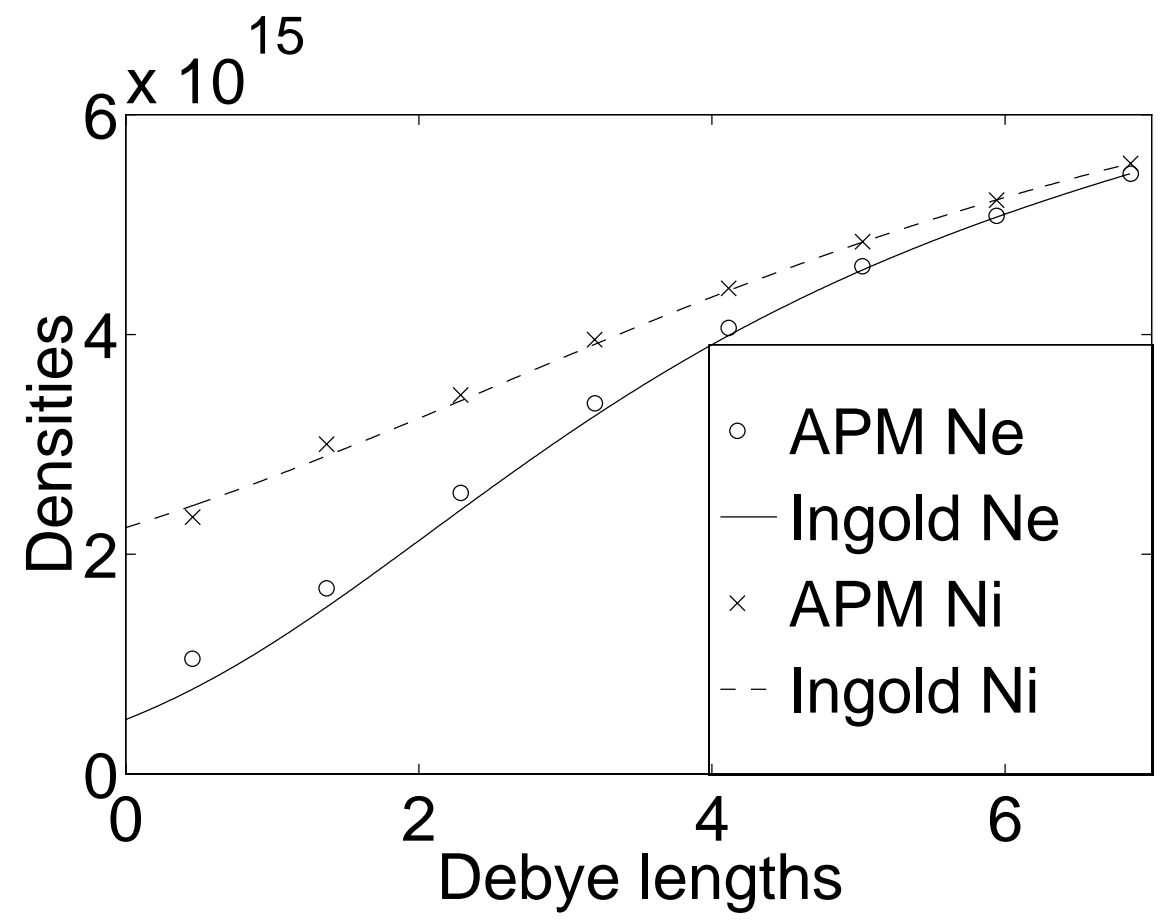

Figure 6.4: Comparison of APM and Ingold sheath model for electron and ion densities. 


\section{Chapter 7}

\section{Applications II : Simulations of}

\section{Inductively Coupled Plasma}

\section{Reactors}

We next consider the application of our method to two problems of interest for ICP processing. Both of these examples demonstrate the importance of using sufficient spatial resolution, which is facilitated by using local grid refinement. A generic ICP reactor is depicted in Figure 2.1. The first simulations examine the effect of different power deposition profiles on the uniformity of ion flux at a wafer surface. These profiles correspond to different RF coil positions and hence address the important engineering issue of optimal coil placement. The second set of simulations considers RF biasing of the wafer surface, in which we examine the effect on ion particle and energy flux.

\subsection{Power Deposition Profile Effects}

Along with selectivity and etch rate, spatial uniformity is a critical process parameter. A uniform etch profile requires a uniform ion flux. One of the main controls over the ion flux is 


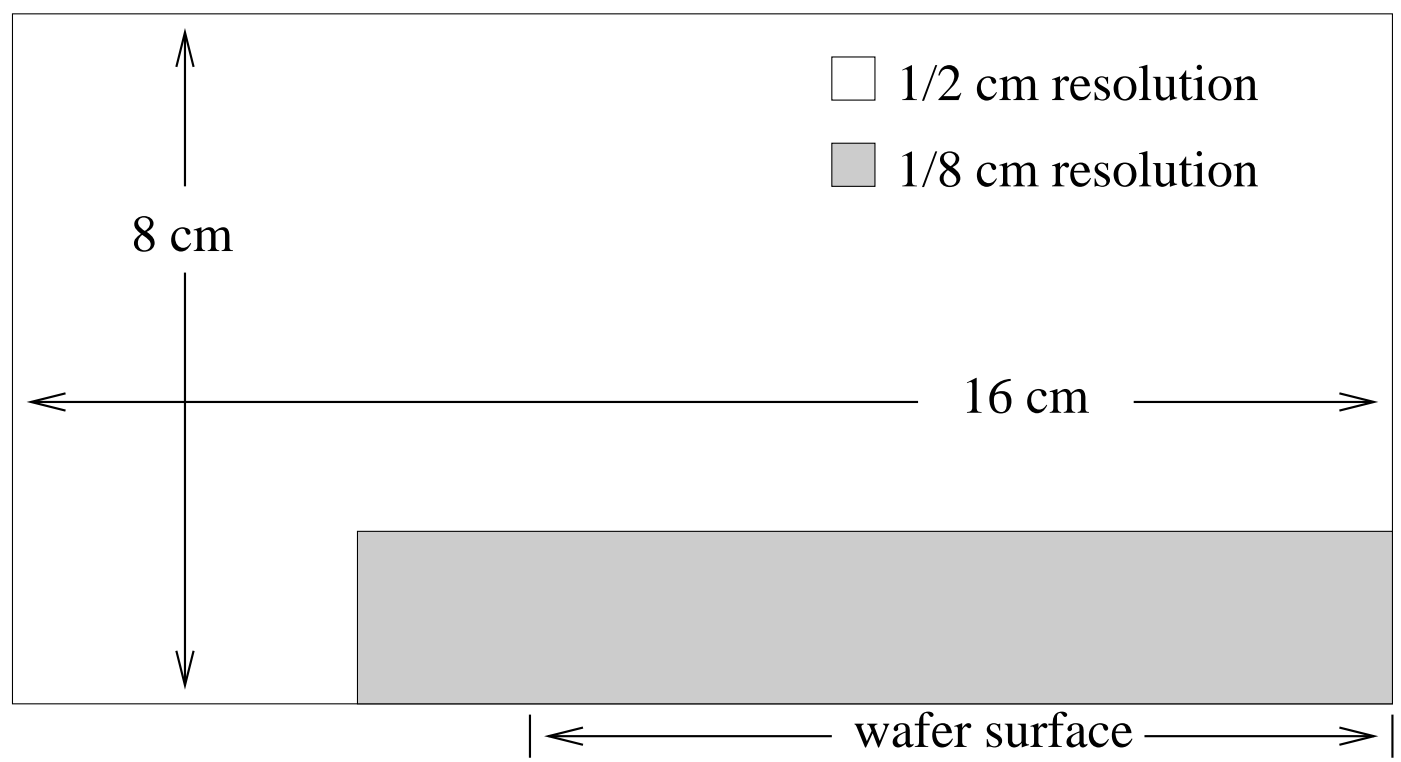

Figure 7.1: Computational domain for the locally refined calculations used to study power deposition effects.

the power deposition within the plasma. The input power drives up the electron temperature and ionization rate. This results in the production of ions which ultimately bombard the wafer or the chamber walls. In this section we examine the effects of coil positions (and hence power deposition) on ion flux uniformity at a wafer surface.

The dimensions and grid structure used in these simulations is shown in Figure 7.1. In these simulations, the time-averaged RF power profile is based on the location of driving coils and an assumed exponential fall off for the power within the plasma. The model does not resolve the inductive fields, nor are the effects of density variations on the power profiles in the plasma considered. Figure 7.2 shows the relation between the assumed reactor coil positions and the resulting power deposition profiles. In each case, the coil power was assumed to fall off with a $1 \mathrm{~cm}$ skin depth normalized to 1000 watts total power. 

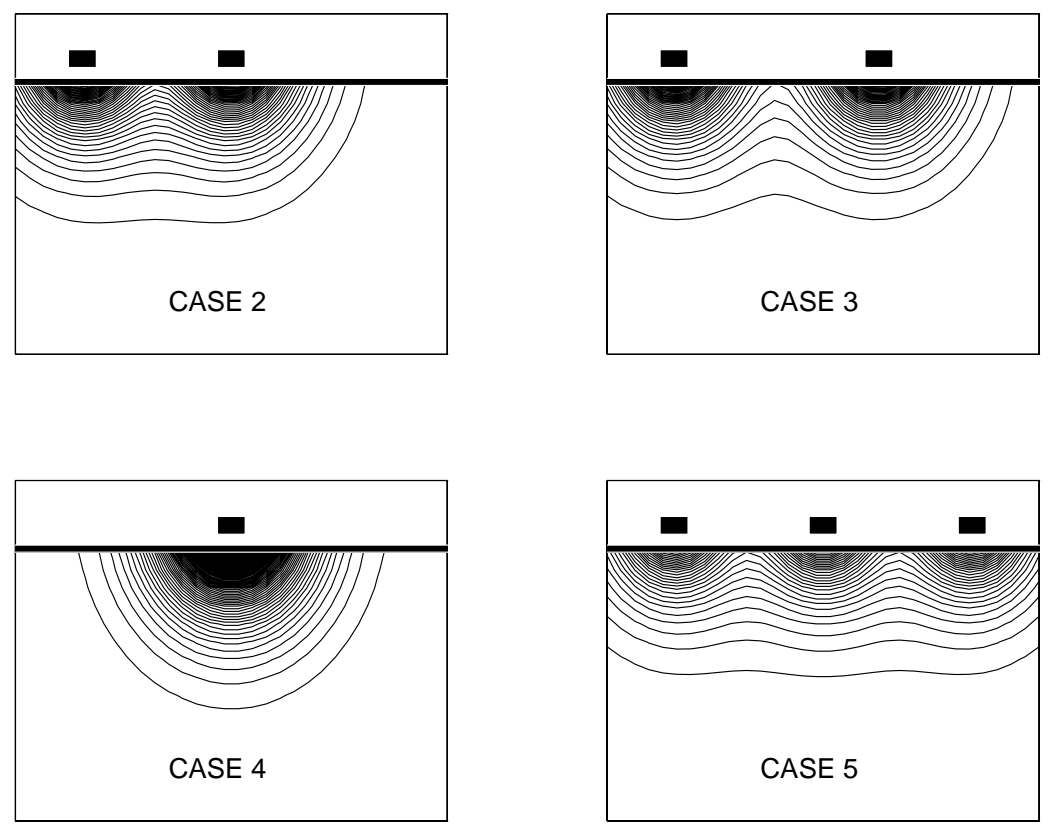

Figure 7.2: Coil positions and power density contours for power deposition study (uniform power density case not shown). 


\subsubsection{Results}

The resulting ion fluxes at the wafer surface were compared for these 5 cases using both a relatively coarse uniform grid and a grid with local refinement near the wafer surface. The uniformity at the wafer surface was measured and is shown for all 5 cases in Figure 7.4.

It is interesting to compare the results for two of these cases, labeled 2 and 3 . Using only the coarse grid solutions, case 3 appears to be a far superior coil configuration. Subsequent refined calculations, however, show that cases 2 and 3 were very similar and that 2 was in fact superior (based on the uniformity figure of merit). This is shown in Figure 7.3. Although total ion flux is not sensitive to grid spacing, this simulation suggests that the exact spatial distribution is.

Results for the best case 2 and case 3 are shown in Figures 7.5 through 7.10.

\subsection{Wafer Biasing Effects}

One of the key advantages to inductively coupled plasma reactors is the ability to independently control ion flux and energy. Capacitively coupled plasmas have an electron production rate dependent on the driving potential gradients. High potential gradients required for high densities often result in very high energy ions bombarding the wafer surface. This is undesirable for some processing steps. In many ICP reactors, the chuck (or platten) holding the semiconductor wafer has an applied RF potential bias applied to it. This has the effect of increasing the average ion energy at the wafer for the same total ion flux. The driving RF coils dump energy into the plasma, but the resulting fields are not seen by the wafer which is many skin depths away. Similarly, the bias field at the wafer does not effect the bulk plasma fields.

In order to quantify these effects and determine their dependence on grid resolution, APM was used to study the effect of RF wafer biasing on the ion flux and energy impinging on the wafer surface. The geometry and grid structure for these calculations is shown in Figure 7.13. Three calculations were performed corresponding to $13.56 \mathrm{MHz}$ bias potentials of 0,10 and 20 volts (peak). 

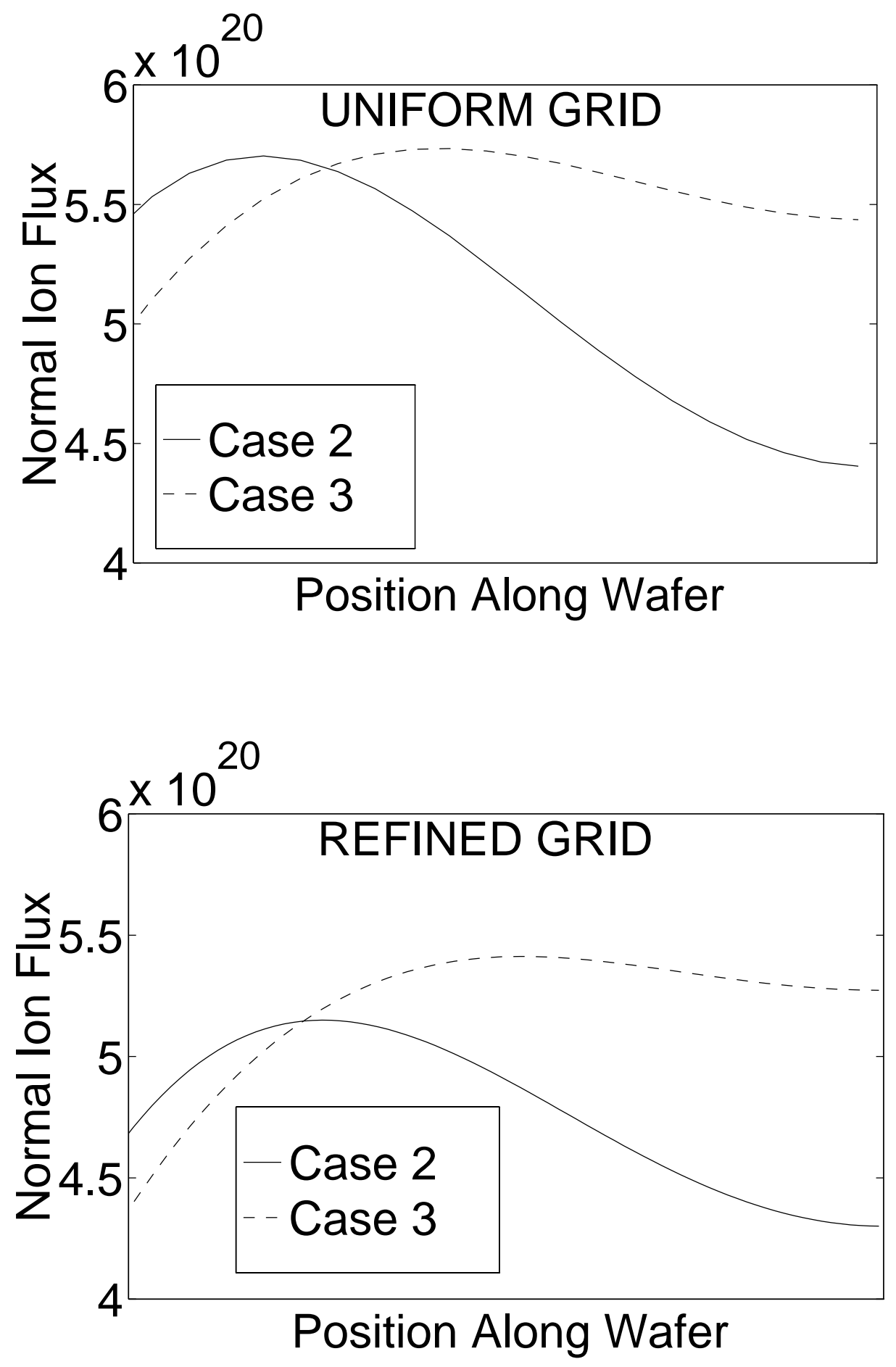

Figure 7.3: Coarse, uniform solution for coil placements 2 and 3 

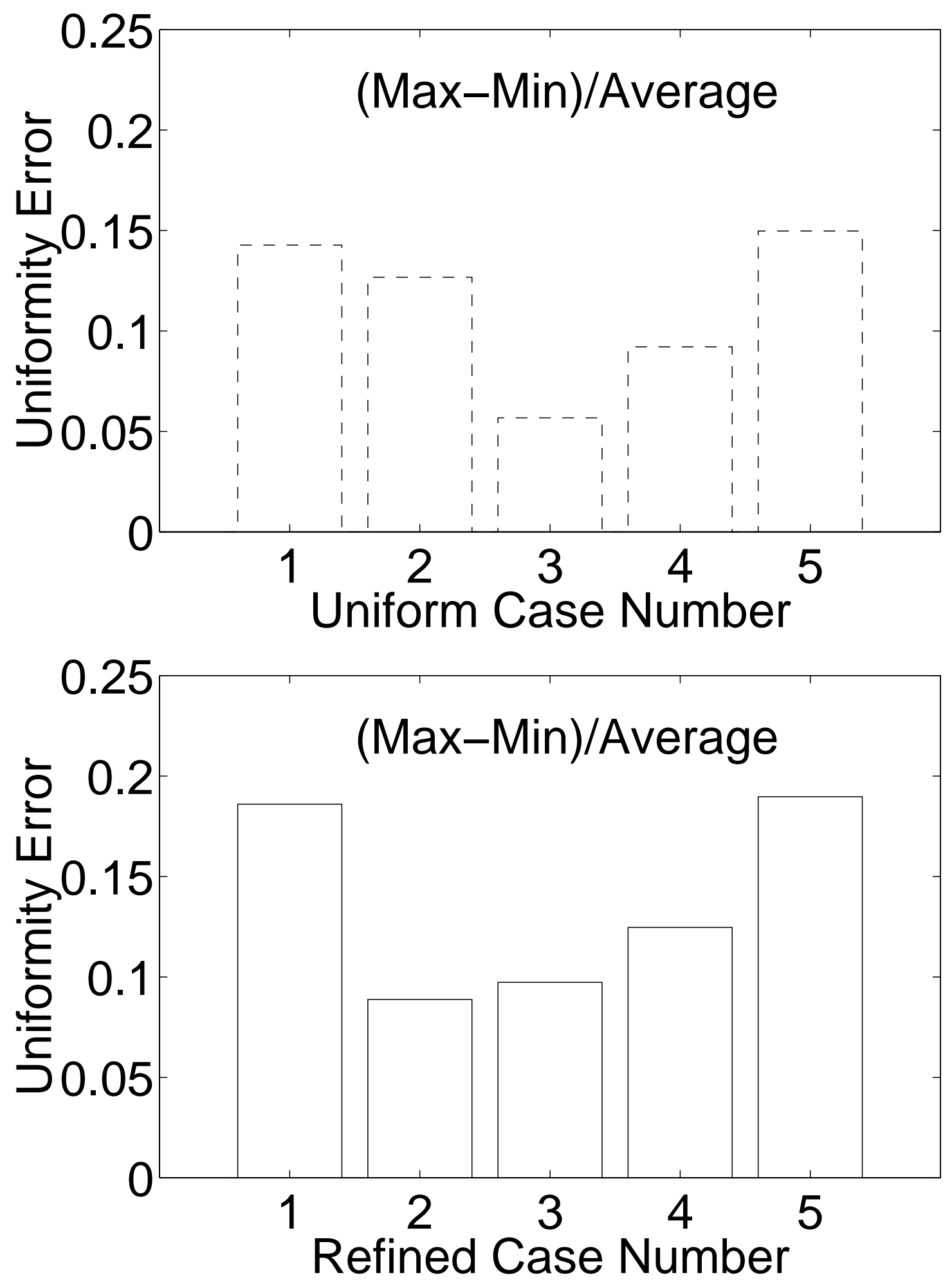

Figure 7.4: Wafer flux uniformity error for refined and uniform grids. 


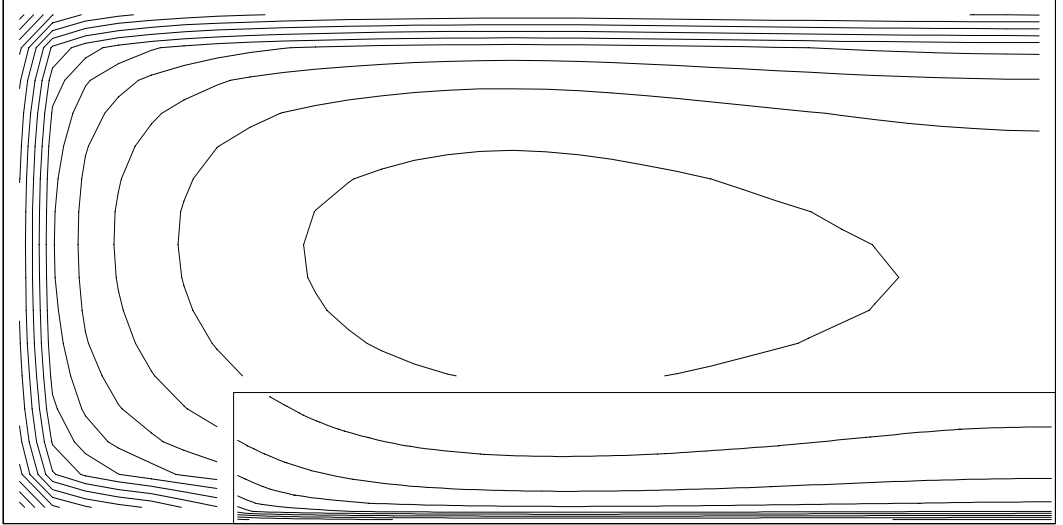

Figure 7.5: Potential contours for case 2.

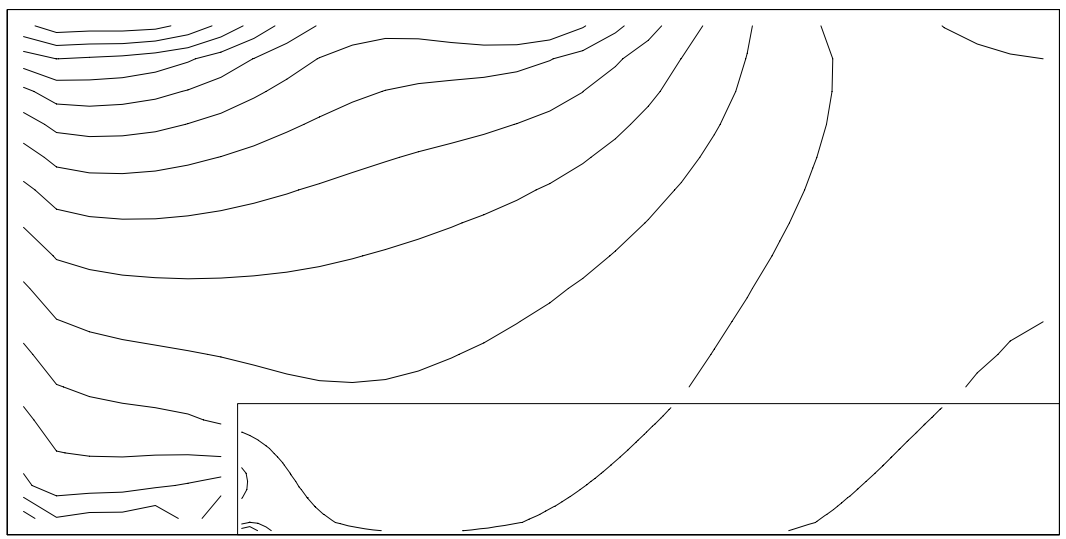

Figure 7.6: Electron temperature contours for case 2.

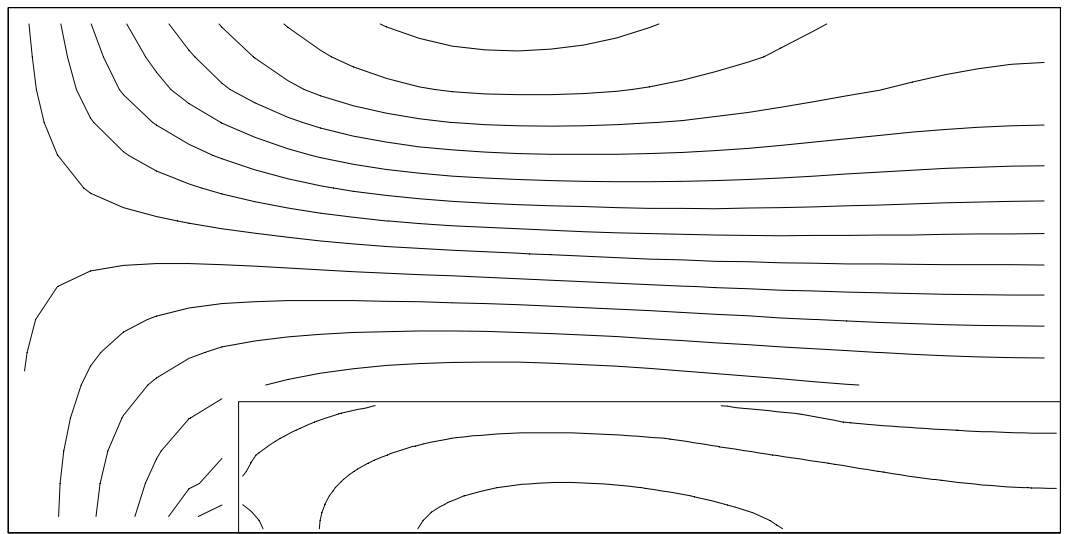

Figure 7.7: Vertical flux contours for case 2. 


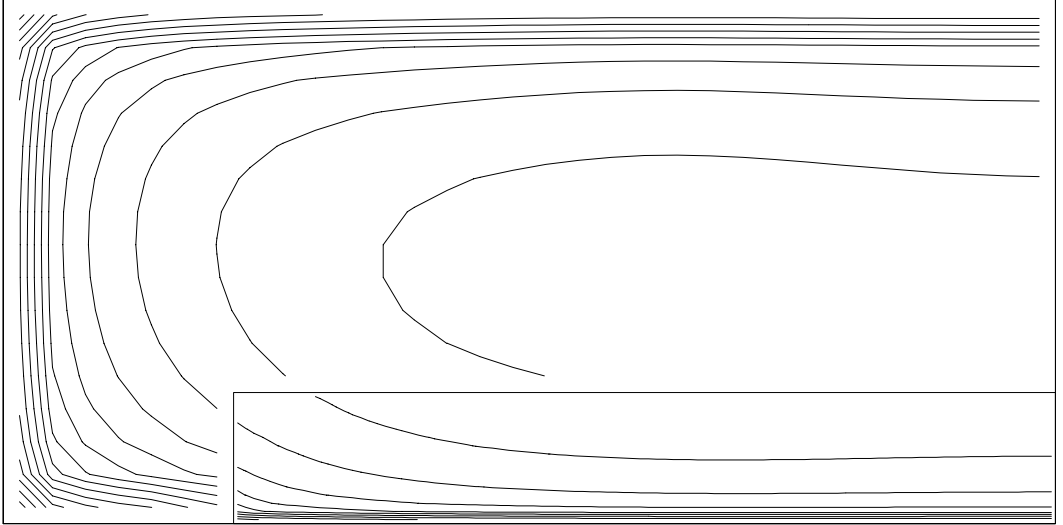

Figure 7.8: Potential contours for case 3.

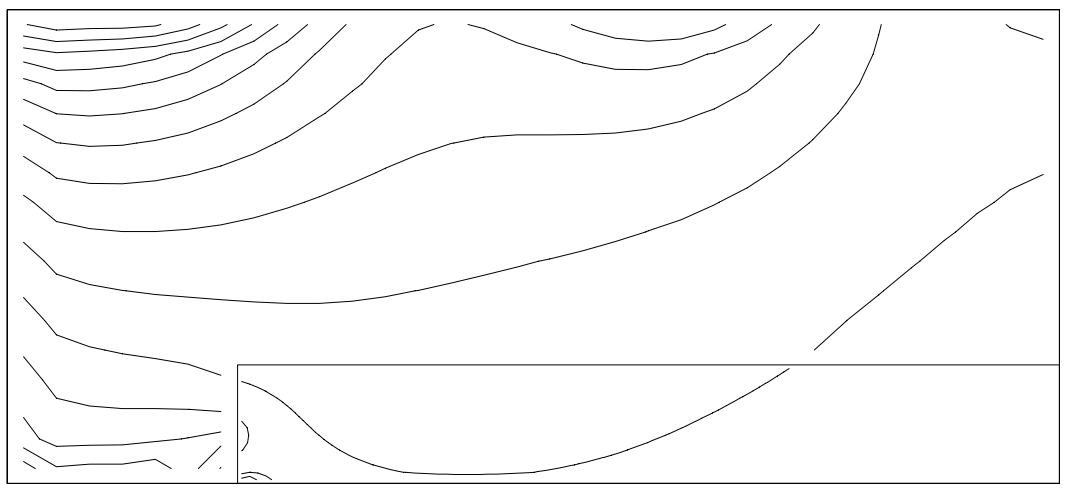

Figure 7.9: Electron temperature contours for case 3.

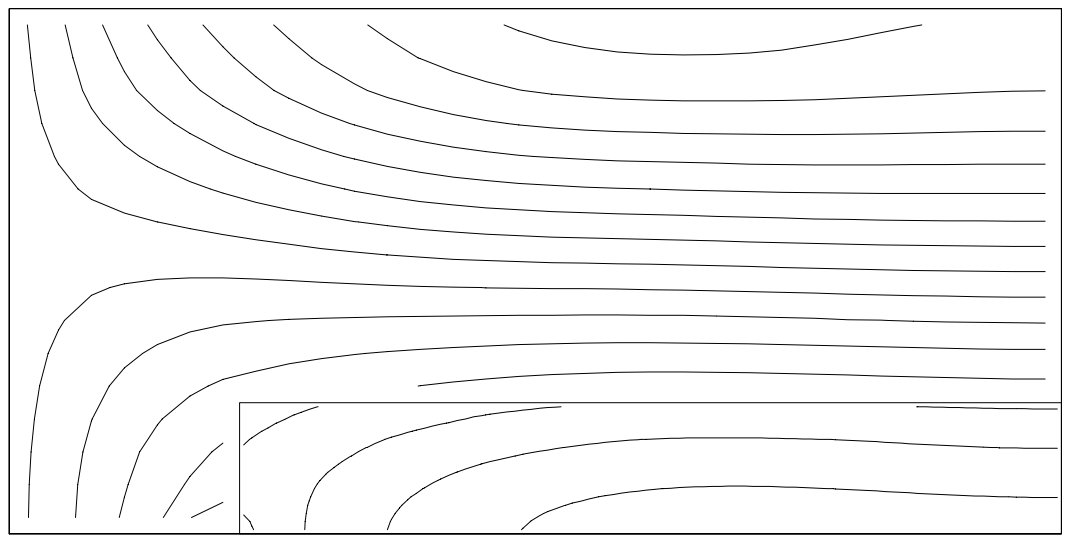

Figure 7.10: Vertical flux contours for case 3. 
Each of these was calculated on a relatively coarse, uniform grid and a locally refined grid. The locally refined case was integrated to steady state on a coarse grid, at which point a refined level was added. This new grid was then also integrated to steady state. The refined grid included regions near the domain boundaries, both those representing the wafer surface and the chamber walls. A complete set of fine, uniform grid calculations were not performed. However, as a check on the adaptive code, one was performed for the 20 volt bias case and found to agree closely with the locally refined case. All cases used a uniform power density of 0.1 watts $/ \mathrm{cm}^{3}$.

\subsubsection{Results}

The weak coupling of the bias potential to the bulk behavior is illustrated in Figures 7.11 and 7.12. In the first two, the time averaged potential from the 20 volt bias case is compared to the potential with no bias. They are significantly different due to variations in the sheath during the cycle. On the other hand, the next two plots show the electron densities for the same calculations. They are quite similar, with peak densities agreeing to within $2 \%$ while the peak potential variation is more than $35 \%$. Ultimately the total flux to the wafer surface will be driven by the density while the energy is strongly influenced by the potential gradients near the wafer surface.

This behavior is displayed in Figures 7.14 through 7.17. As expected, the ion flux was insensitive to the bias potential as well as grid resolution effects. The variation due to the bias was less than $2.1 \%$ and that of the grid resolution was less than $3.4 \%$. Note how this contrasts with the previously discussed power deposition study in which power deposition profiles greatly effected the ion flux uniformity. The ion energy changed significantly at higher bias potentials, however. The 20 volt bias resulted in higher ion energies of up to $61.4 \%$. The higher resolution calculations differed significantly from the corresponding uniform calculations as well. These grid resolution effects accounted for up to $17.0 \%$ variations in the ion energy. This suggests that an accurate computational study of RF biasing effects requires high spatial accuracy in the wafer region to properly resolve ion energy profiles. 

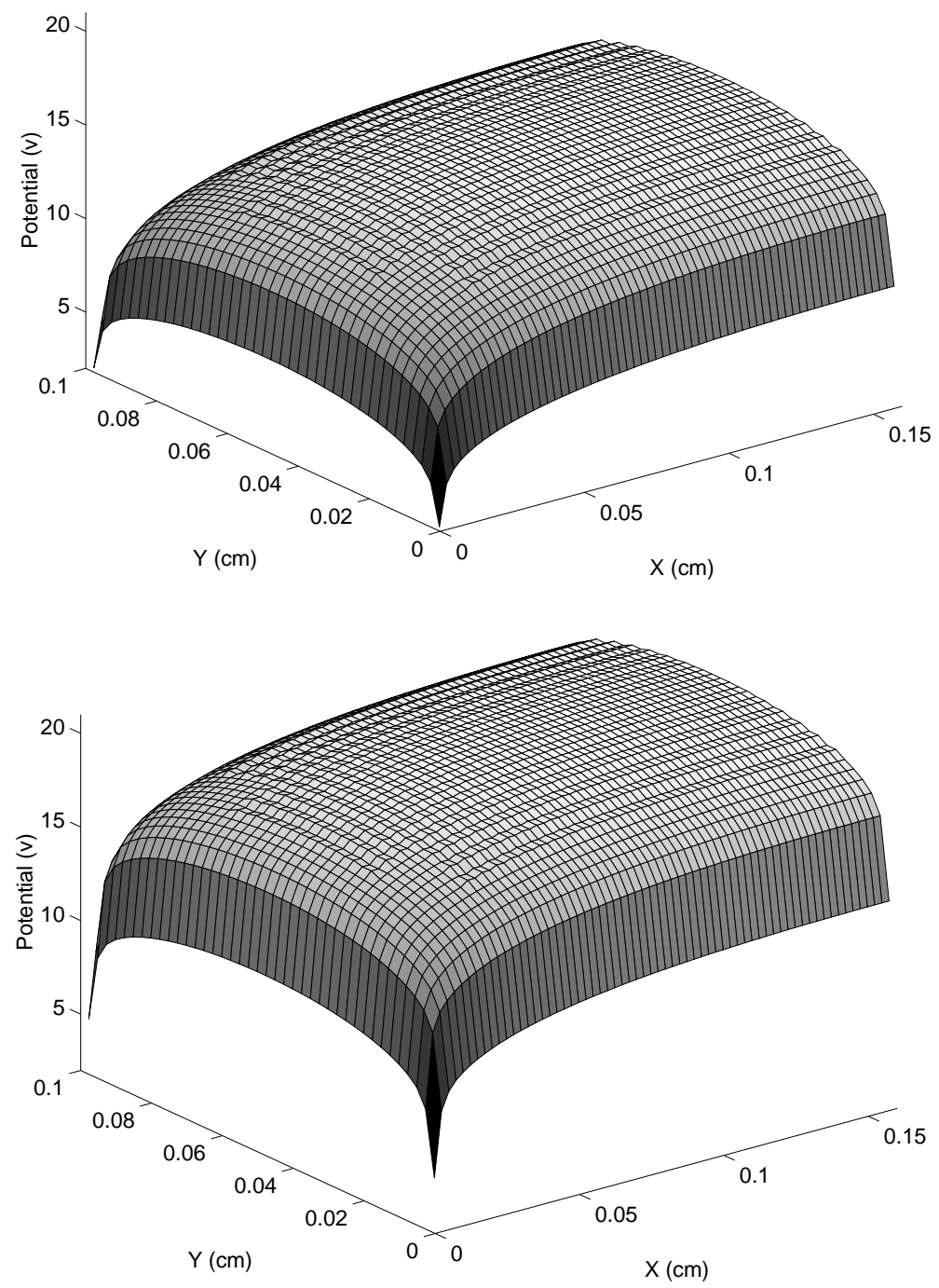

Figure 7.11: Time averaged potentials for zero bias (above) and 20 volt RF bias (below) cases. 

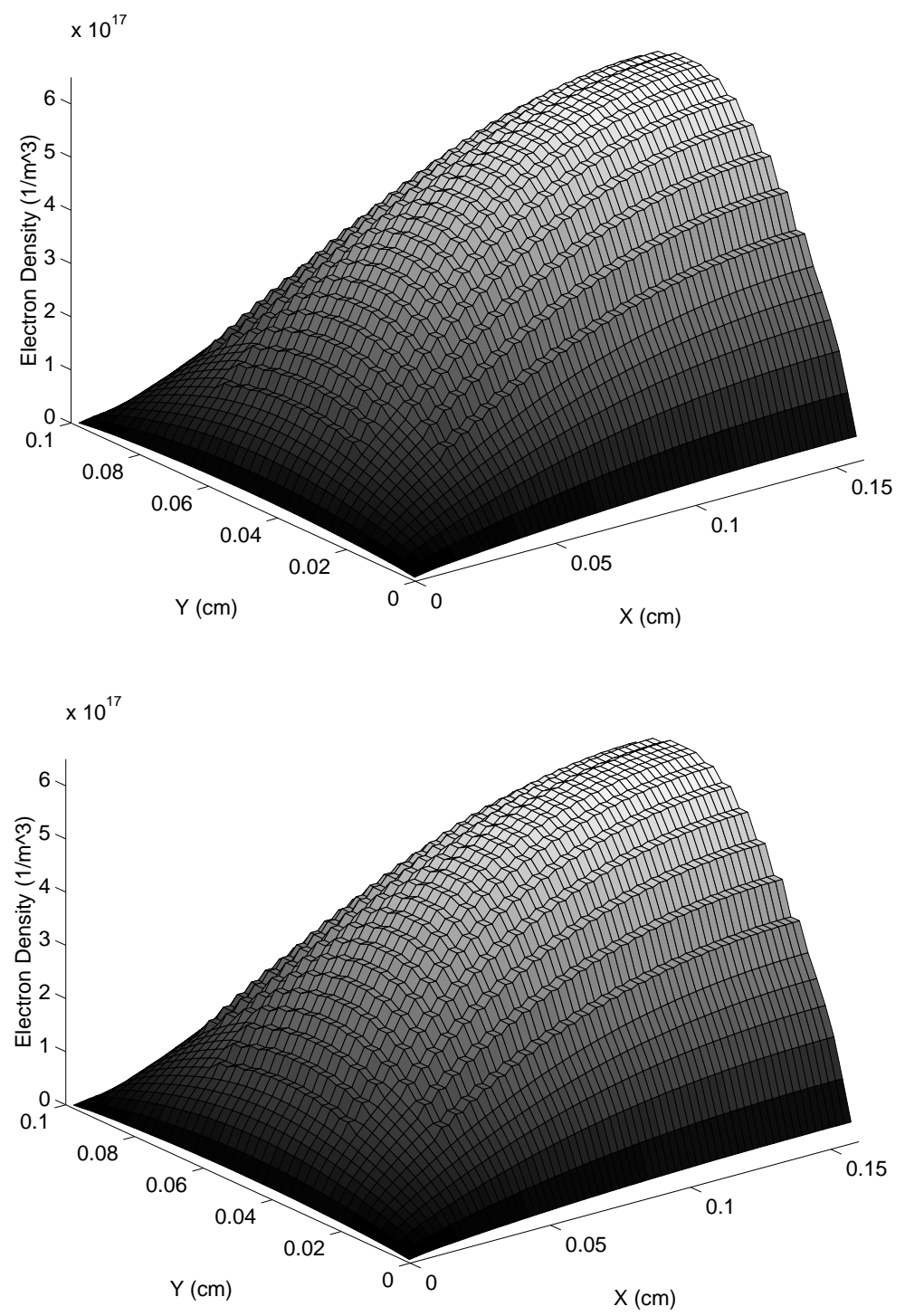

Figure 7.12: Time averaged electron density for zero bias (above) and 20 volt RF bias (below) cases. 


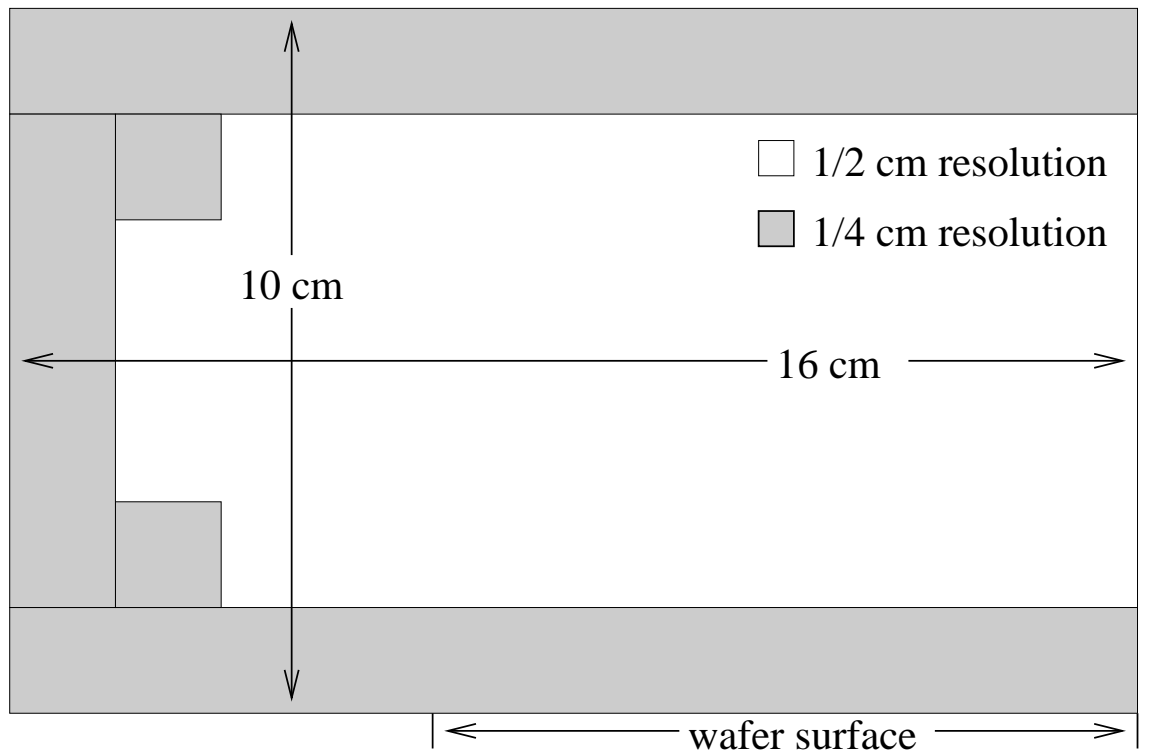

Figure 7.13: Computational domain used in the locally refined calculations as part of the wafer bias study.

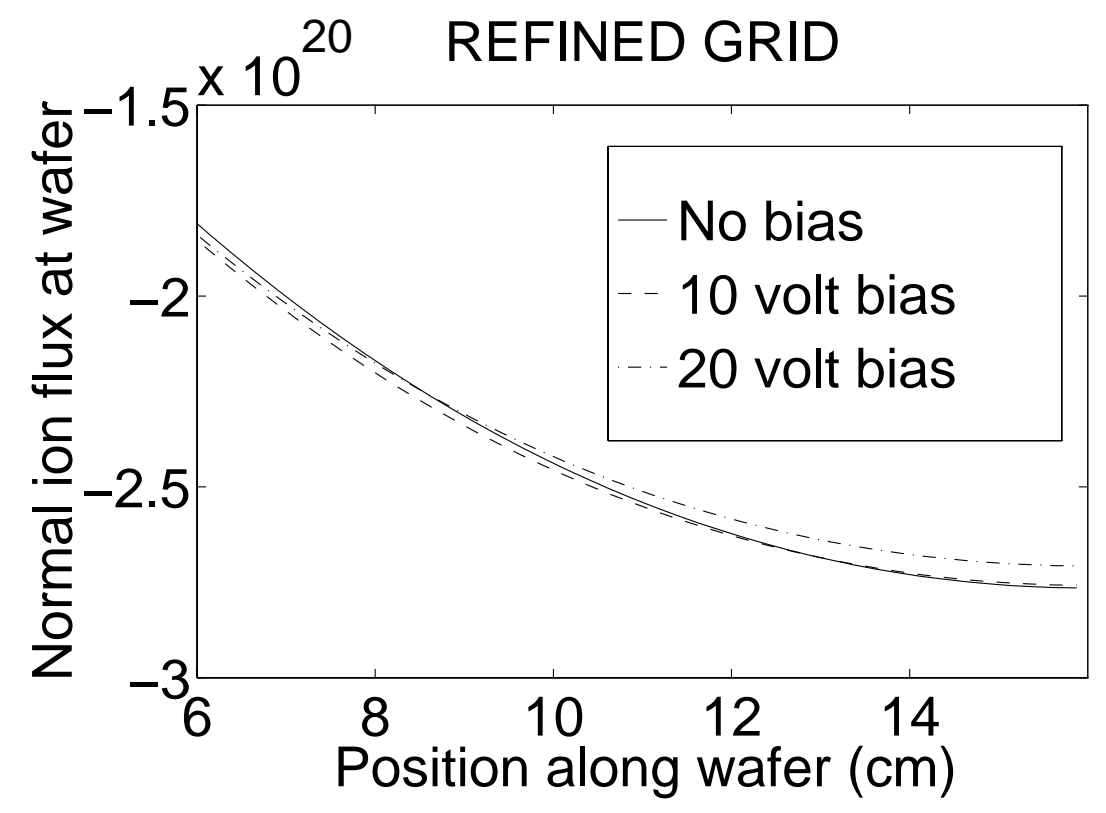

Figure 7.14: Ion normal flux for locally refined grid calculations. 


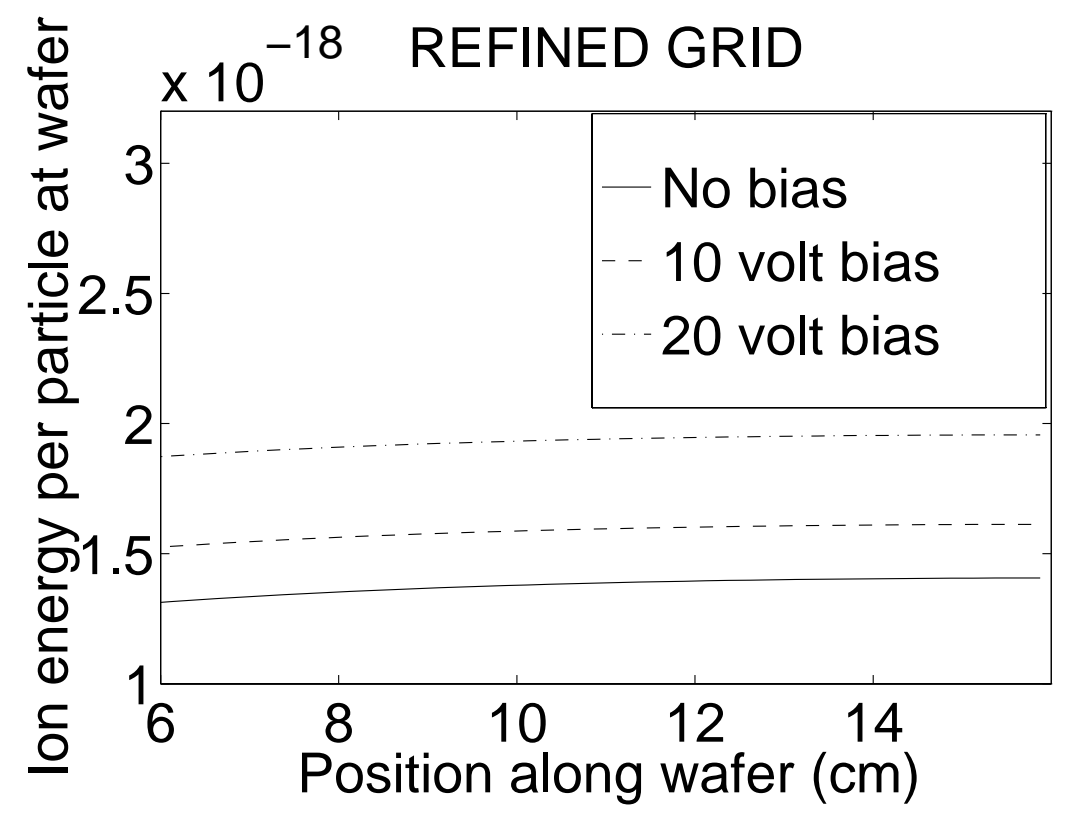

Figure 7.15: Ion energy per particle for locally refined grid calculations.

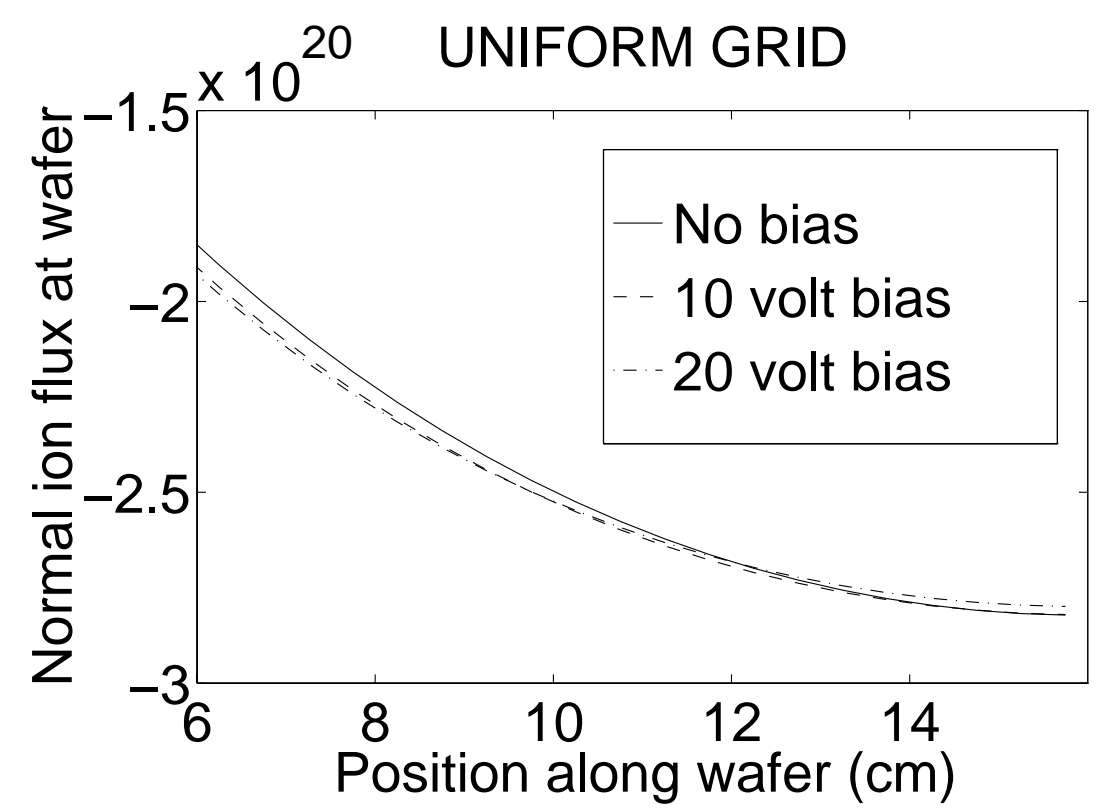

Figure 7.16: Ion normal flux for uniform and locally refined grid calculations. 


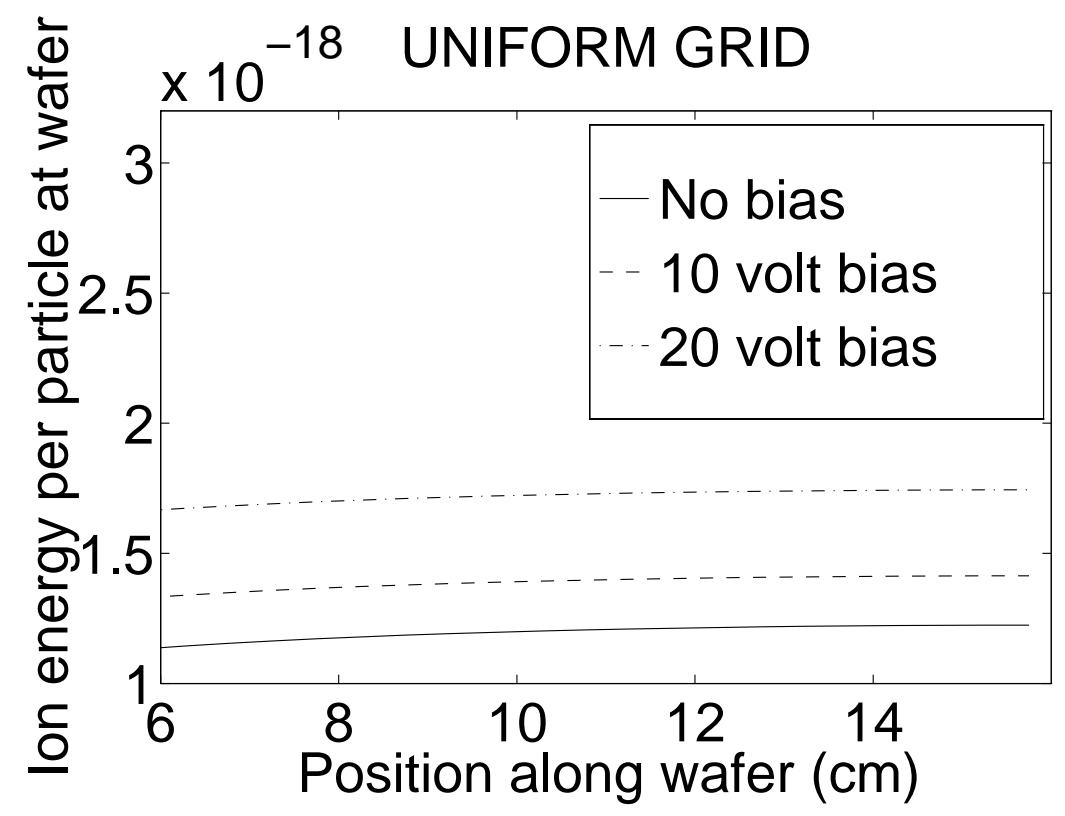

Figure 7.17: Ion energy per particle for uniform and locally refined grid calculations. 


\section{Chapter 8}

\section{Conclusion}

Rather than simply repeat the introduction, we will briefly summarize the work done in this thesis and the remainder of the chapter will be devoted to a discussion of future work and speculation as to how this work may prove most useful.

Let us review what has been done. We have described a new numerical method for plasma simulation. Calculations have been presented which show that the method is accurate and suggest the regimes in which the method provides savings in CPU time and memory requirements. A steady state simulation of a four centimeter domain was modeled with sheath scale $(150 \mu \mathrm{m})$ resolution using only 40 grid points. Simulations of semiconductor processing equipment have been performed which imply the usefulness of the method for engineering applications. It is the author's opinion that these accomplishments represent a significant contribution to plasma simulation and the efficient numerical solution of certain systems of non-linear, partial differential equations.

More work needs to be done, however, for the algorithm to be of practical use in an engineering environment. Despite our success at avoiding the dielectric relaxation timestep restriction, the algorithm is still conditionally stable and requires timesteps which are relatively small. This represents a prohibitive runtime for steady state solutions on high resolution grids. Current research suggests that these limitations may be overcome and the use of much larger timesteps will be possible 
in the future.

While two-dimensional simulations are the rule today, three-dimensional codes will be the trend in the near future. This should be an area in which this method shines. Fast algorithms will be imperative for demanding three-dimensional problems. Another reason for optimism is that the extension of the method to three-dimensions is straightforward. The ability to include realistic geometry within the plasma is another necessary feature. Unfortunately, this is a more challenging task. As mentioned in the introduction, this is an active area of research and progress is being made.

The physical model should also be improved. The goal of this work was to demonstrate the fruitful application of AMR techniques to plasma processing simulation. That being the case, the addition of complicated chemistry or other effects would have been beneficial, but not critical. This is not the case for a production code in a manufacturing environment, however. A more general electromagnetic capability would be desirable as well.

Finally, we should address the issue of the applicability of fluid models to these systems. For the parameters used in this work, the two-fluid model should be a very accurate representation within the bulk plasma. As we approach the sheath and pre-sheath regions at boundaries, the validity of the fluid models become less clear. It is certainly the case that the region for which the fluid model is valid is extended through the use of high resolution grids. Perhaps this algorithm would be useful as part of a hybrid code in which sheath regions were treated in some kinetic fashion. Considering the computational penalty associated with kinetic codes, the extended region of validity of the high resolution fluid models would surely be appreciated.

In conclusion, we have made progress in the development of simulation tools for plasma based semiconductor manufacturing. It is satisfying to know that while computational scientists all over the world are developing computer codes while wishing for faster computer chips, our computational research is doing something about it. 


\section{Appendix A}

\section{Linearized Stability Analysis of the}

\section{Coupled Electron/Potential}

\section{Update Scheme}

\section{A.1 Introduction}

The APM code solves a set of coupled non-linear partial differential equations. These equations and the discretizations used to solve them are too complicated for a standard stability analysis. Some knowledge about the stability of the method is desired however. In this section, the coupled electron and potential update is linearized and its stability is analyzed. This part of the algorithm is the stiffest and the one for which timestep restrictions are most likely to occur. 


\section{A.2 Linearized Model Equations}

Only one space dimension is considered. The ion density and the electron temperature are assumed to vary slowly on the time scale of interest and are assumed constant. Ionization is neglected as well. This leaves the following pair of equations.

$$
\begin{gathered}
\frac{\partial n_{e}}{\partial t}+\mu \frac{\partial}{\partial x}\left(n_{e} \frac{\partial \phi_{e}}{\partial x}\right)=\alpha \frac{\partial^{2} n_{e}}{\partial x^{2}} \\
\frac{\epsilon_{0}}{e} \frac{\partial}{\partial t} \frac{\partial^{2} \phi_{e}}{\partial x^{2}}=\frac{\partial n_{e}}{\partial t}
\end{gathered}
$$

Where $\mu=|q| / m_{e} \nu_{e n}$ is the mobility and $\alpha=k T_{e} / m_{e} \nu_{e n}$ is the diffusivity.

The potential and electron density are still non-linearly coupled. To linearize, assume that $n_{e}$ and $\partial \phi_{e} / \partial x$ are the sum of a large constant part and a small perturbation which varies in time and space.

$$
\begin{aligned}
n_{e} & =n_{0}+n(x, t) \\
\frac{\partial \phi_{e}}{\partial x} & =\frac{\partial \phi_{0}}{\partial x}+\frac{\partial \phi}{\partial x}(x, t)
\end{aligned}
$$

Without loss of generality, we assume that $\partial \phi_{0} / \partial x$ is non-negative. Substituting these expressions into (A.1) and (A.2) and discarding quadratic and higher terms for the perturbations, we get the following pair of linear equations.

$$
\begin{gathered}
\frac{\partial n}{\partial t}+\mu n_{0} \frac{\partial^{2} \phi}{\partial x^{2}}+\mu \frac{\partial \phi_{0}}{\partial x} \frac{\partial n}{\partial x}=\alpha \frac{\partial^{2} n}{\partial x^{2}} \\
\frac{\epsilon_{0}}{e} \frac{\partial}{\partial t} \frac{\partial^{2} \phi}{\partial x^{2}}=\frac{\partial n}{\partial t}
\end{gathered}
$$

If we substitute (A.3) into (A.4) we arrive at an equivalent system which is the one we will study. 


$$
\begin{aligned}
\frac{\partial n}{\partial t} & =-\mu n_{0} \frac{\partial^{2} \phi}{\partial x^{2}}-\mu \frac{\partial \phi_{0}}{\partial x} \frac{\partial n}{\partial x}+\alpha \frac{\partial^{2} n}{\partial x^{2}} \\
\frac{\epsilon_{0}}{e} \frac{\partial}{\partial t} \frac{\partial^{2} \phi}{\partial x^{2}} & =-\mu n_{0} \frac{\partial^{2} \phi}{\partial x^{2}}-\mu \frac{\partial \phi_{0}}{\partial x} \frac{\partial n}{\partial x}+\alpha \frac{\partial^{2} n}{\partial x^{2}}
\end{aligned}
$$

These are pointwise equations. Because our formulation of the algorithm is for the cell averages, we would like to have equations for the cell averages of $n$ and $\phi$. To do this, we introduce the cell averaging operator.

$$
\langle F(x, t)\rangle \equiv \frac{1}{\Delta t \Delta x} \int_{t^{n}}^{t^{n+1}} \int_{x^{i-1 / 2}}^{x^{i+1 / 2}} F(x, t) d x d t
$$

We apply this operator to the above equations and henceforth interpret the variables as cell averages. This gives a general form for the discretizations

$$
\begin{aligned}
\frac{n_{i}^{n+1}-n_{i}^{n}}{\Delta t} & =-\mu n_{0}\left\langle\frac{\partial^{2} \phi}{\partial x^{2}}\right\rangle-\mu \frac{\partial \phi_{0}}{\partial x}\left\langle\frac{\partial n}{\partial x}\right\rangle+\alpha\left\langle\frac{\partial^{2} n}{\partial x^{2}}\right\rangle \\
\frac{\epsilon_{0}}{e} \frac{\partial^{2} \phi_{i}^{n+1} / \partial x^{2}-\partial^{2} \phi_{i}^{n} / \partial x^{2}}{\Delta t} & =-\mu n_{0}\left\langle\frac{\partial^{2} \phi}{\partial x^{2}}\right\rangle-\mu \frac{\partial \phi_{0}}{\partial x}\left\langle\frac{\partial n}{\partial x}\right\rangle+\alpha\left\langle\frac{\partial^{2} n}{\partial x^{2}}\right\rangle
\end{aligned}
$$

where subscripts refer to cell locations and superscripts refer to time levels. The left hand side of the above equations become simple temporal differences of cell averages. The quantities in brackets on the right hand side represent numerical quadratures for the spatial derivatives. It is interesting to note that many different update schemes can be written in this form, differing only in the choice of quadratures for these terms.

\section{A.3 APM Algorithm}

The APM algorithm introduces a number of temporary quantities used in the final update of the state variables. In one dimension, some of them are :

$\hat{n}_{i}^{n+1} \equiv$ Cell average density approximation at time $t^{n+1}$.

$\hat{n}_{i+1 / 2}^{n+1 / 2} \equiv$ Right edge density approximation at time $t^{n+1 / 2}$. 
$\hat{n}_{i-1 / 2}^{n+1 / 2} \equiv$ Left edge density approximation at time $t^{n+1 / 2}$.

The following diagram illustrates the location of these quantities in time and space.

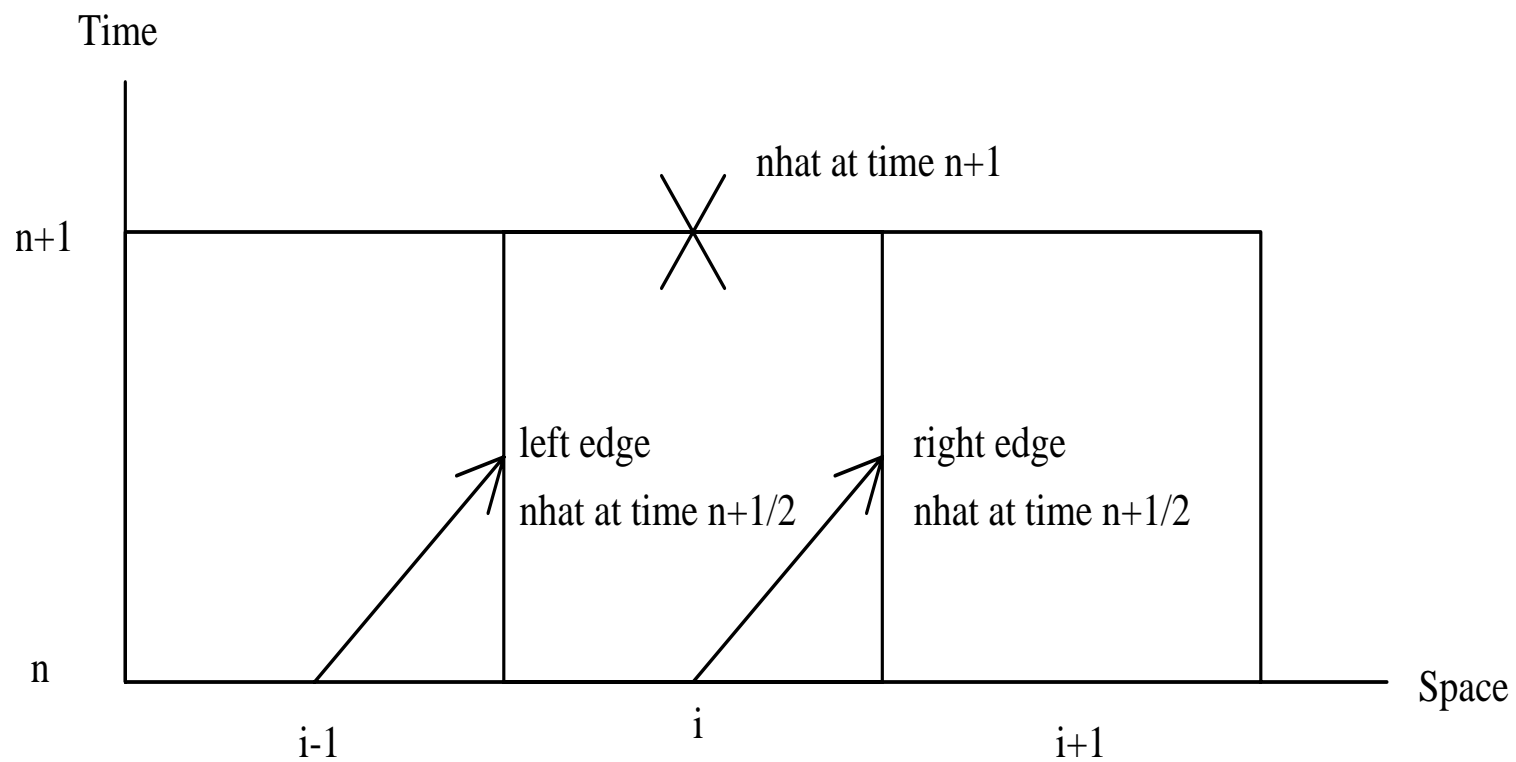

These explicitly calculated quantities are used to improve the accuracy of the update scheme without introducing any undesirable coupling between the state variables. First, $\hat{n}_{i+1 / 2}^{n+1 / 2}$ and $\hat{n}_{i-1 / 2}^{n+1 / 2}$ are explicit, upwind Taylor expansions for the edge densities at time $t^{n+1 / 2}$ given by

$$
\begin{gathered}
\hat{n}_{i+1 / 2}^{n+1 / 2}=n_{i}^{n}+\frac{\Delta x}{2}\left(1-\frac{\Delta t \mu \frac{\partial \phi_{0}}{\partial x}}{\Delta x}\right)\left(\frac{n_{i+1}^{n}-n_{i-1}^{n}}{2 \Delta x}\right)+ \\
+\frac{\Delta t}{2 \Delta x^{2}}\left(\alpha\left(n_{i+1}^{n}-2 n_{i}^{n}+n_{i-1}^{n}\right)-\mu n_{0}\left(\phi_{i+1}^{n}-2 \phi_{i}^{n}+\phi_{i-1}^{n}\right)\right) \\
\hat{n}_{i-1 / 2}^{n+1 / 2}=n_{i-1}^{n}+\frac{\Delta x}{2}\left(1-\frac{\Delta t \mu \frac{\partial \phi_{0}}{\partial x}}{\Delta x}\right)\left(\frac{n_{i}^{n}-n_{i-2}^{n}}{2 \Delta x}\right)+ \\
+\frac{\Delta t}{2 \Delta x^{2}}\left(\alpha\left(n_{i}^{n}-2 n_{i-1}^{n}+n_{i-2}^{n}\right)-\mu n_{0}\left(\phi_{i}^{n}-2 \phi_{i-1}^{n}+\phi_{i-2}^{n}\right)\right)
\end{gathered}
$$

Second, $\hat{n}_{i}^{n+1}$ is implicitely calculated by

$$
\frac{\hat{n}^{n+1}-n^{n}}{\Delta t}=\alpha\left(\frac{\hat{n}_{i+1}^{n+1}-2 \hat{n}_{i}^{n+1}+\hat{n}_{i-1}^{n+1}}{\Delta x^{2}}\right)
$$




$$
-\mu n_{0}\left(\frac{\phi_{i+1}^{n}-2 \phi_{i}^{n}+\phi_{i-1}^{n}}{\Delta x^{2}}\right)-\frac{\mu}{2} \frac{\partial \phi_{0}}{\partial x}\left(\frac{\hat{n}_{i+1 / 2}^{n+1 / 2}-\hat{n}_{i-1 / 2}^{n+1 / 2}}{\Delta x}\right)
$$

With these approximations, we can state the method used to obtain the quadratures mentioned above.

First, the potential must be solved implicitly in order avoid very strict timestep restrictions.

The average potential Laplacian is then approximated using a Backward Euler discretization

$$
\left\langle\frac{\partial^{2} \phi}{\partial x^{2}}\right\rangle \approx \frac{\phi_{i+1}^{n+1}-2 \phi_{i}^{n+1}+\phi_{i-1}^{n+1}}{\Delta x^{2}}
$$

The hyperbolic term is explicitely evaluated by upwind Taylor series approximations at the cell edges at the half time level

$$
\left\langle\frac{\partial n}{\partial x}\right\rangle \approx \frac{1}{2}\left(\frac{\hat{n}_{i+1 / 2}^{n+1 / 2}-\hat{n}_{i-1 / 2}^{n+1 / 2}}{\Delta x}\right) .
$$

For the diffusive term, we also apply Backward Euler

$$
\left\langle\frac{\partial^{2} n}{\partial x^{2}}\right\rangle \approx \alpha\left(\frac{\hat{n}_{i+1}^{n+1}-2 \hat{n}_{i}^{n+1}+\hat{n}_{i-1}^{n+1}}{\Delta x^{2}}\right) .
$$

\section{A.3.1 Fourier Analysis}

Fourier (or VonNeumann) analysis is used to look at the linear stability of this method. The equations are transformed from configuration space ( $x$ variable) to wavenumber space ( $k$ variable). The transformed variables, denoted by tildes, are functions of $k$ and $t$.

The growth of the transformed equations is equivalent to the growth of the real equations, in that Parseval's theorem holds for the discrete Fourier transform

$$
\int_{x \min }^{x \max }|f(x, t)|^{2} d x=\int_{-\infty}^{\infty}|\tilde{f}(k, t)|^{2} d k
$$

Having transformed the equations, we can analyze the APM algorithm for stability induced timestep restrictions. First, the transformed equation for the predicted electron densities is

$$
\frac{\tilde{\hat{n}}^{n+1}-\tilde{n}^{n}}{\Delta t}+\left(\frac{Z_{l a p} \mu n_{0}}{\Delta x^{2}}\right) \tilde{\phi}^{n}+\left(\frac{Z_{u p} \mu \frac{\partial \phi_{0}}{\partial x}}{\Delta x}\right) \tilde{n}^{n}=\left(\frac{Z_{l a p} \alpha}{2 \Delta x^{2}}\right)\left(\tilde{\hat{n}}^{n+1}+\tilde{n}^{n}\right)
$$


the modified Poisson equation is

$$
\left(\frac{Z_{l a p} \epsilon_{0}}{\Delta t \Delta x^{2} e}\right)\left(\tilde{\phi}^{n+1}-\tilde{\phi}^{n}\right)=-\left(\frac{Z_{l a p} \mu n_{0}}{2 \Delta x^{2}}\right)\left(\tilde{\phi}^{n+1}+\tilde{\phi}^{n}\right)-\left(\frac{Z_{u p} \mu \frac{\partial \phi_{0}}{\partial x}}{\Delta x}\right) \tilde{n}^{n}+\left(\frac{Z_{l a p} \alpha}{2 \Delta x^{2}}\right)\left(\tilde{\hat{n}}^{n+1}+\tilde{n}^{n}\right)
$$

and the continuity equation is

$$
\frac{\tilde{n}^{n+1}-\tilde{n}^{n}}{\Delta t}+\left(\frac{Z_{l a p} \mu n_{0}}{2 \Delta x^{2}}\right)\left(\tilde{\phi}^{n+1}+\tilde{\phi}^{n}\right)+\left(\frac{Z_{u p} \mu \frac{\partial \phi_{0}}{\partial x}}{\Delta x}\right) \tilde{n}^{n}=\left(\frac{Z_{l a p} \alpha}{2 \Delta x^{2}}\right)\left(\tilde{\hat{n}}^{n+1}+\tilde{n}^{n}\right)
$$

where $Z_{l a p}=(\exp (i \omega)-2+\exp (-i \omega)), Z_{u p}=(1-\exp (-i \omega))$ and $\omega=k \Delta x$ are phase factors corresponding to the Laplacian and upwind difference operators, and a unitless angular frequency, respectively. Equation (A.13) can be solved for $\tilde{\hat{n}}^{n+1}$ and this result can be substituted into (A.14) and (A.15). This results in a linear system relating the transformed state variables at the new and old times. The maximum eigenvalue of the resulting matrix determines the stability of the method. If the maximum eigenvalue has absolute value less than 1 , then the method is stable.

These eigenvalues were analyzed using material properties representative of the system of interest. In all cases, the condition on the stability of the method was the usual Courant condition

$$
\Delta t \leq \frac{\Delta x}{V_{0}}
$$

where $V_{0}=\mu \partial \phi_{0} / \partial x$.

The range of parameters studied included $\partial \phi_{0} / \partial x$ from $10^{-1}$ to $10^{5}$ volts per meter, $n_{0}$ from $10^{9}$ to $10^{13}$ particles per cubic centimeter, $k T_{e}$ from 0.2 to $20 \mathrm{eV}$, and $\Delta x$ from 0.00001 to 0.1 meters.

\section{A.4 Simplified Systems}

\section{A.4.1 Cold Electrons}

Some further simplification can be made. One is to ignore the diffusivity, or equivalently, consider cold electrons. In this case, the system to analyze is 


$$
\begin{aligned}
\frac{n_{i}^{n+1}-n_{i}^{n}}{\Delta t} & =-\mu n_{0}\left\langle\frac{\partial^{2} \phi}{\partial x^{2}}\right\rangle-\mu \frac{\partial \phi_{0}}{\partial x}\left\langle\frac{\partial n}{\partial x}\right\rangle \\
\frac{\epsilon_{0}}{e} \frac{\partial^{2} \phi_{i}^{n+1} / \partial x^{2}-\partial^{2} \phi_{i}^{n} / \partial x^{2}}{\Delta t} & =-\mu n_{0}\left\langle\frac{\partial^{2} \phi}{\partial x^{2}}\right\rangle-\mu \frac{\partial \phi_{0}}{\partial x}\left\langle\frac{\partial n}{\partial x}\right\rangle
\end{aligned}
$$

This system proves useful for the investigation of different approximations for the advective term. We would like to compare the stability using the second order method, which utilizes the predicted edge densities given by (A.9-A.10), to that using a first order method. The first order method simply uses the upwind cell centered densities at time $t^{n}$ for the edge predictions.

For both the first and second order cases, the discrete transform was taken and the eigenvalues analyzed. Again, we require that the absolute value of the eigenvalues be less than or equal to one. In both case, maximum growth occurs for the case $\omega=\pi$. In the first order case, the extreme eigenvalue is

$$
\lambda=\frac{\Delta x \epsilon_{0}-2 \epsilon_{0} \Delta t V_{0}}{\Delta x \epsilon_{0}+\Delta x \Delta \operatorname{ten}_{0} \mu}
$$

If we make the following definitions

$$
\begin{aligned}
\Delta t & =c \Delta x / V_{0} \\
\tau_{\text {dielectric }} & =\epsilon_{0} / e \mu n_{0} \\
\tau_{\text {transit }} & =\Delta x / V_{0} \\
\beta & =\tau_{\text {transit }} / \tau_{\text {dielectric }}
\end{aligned}
$$

we can write this eigenvalue as

$$
\lambda=\frac{1-2 c}{1+\beta c}
$$

Down to sheath scales, for parameters of interest, $\beta>>1$. Therefore the first order discretization results in an unconditionally stable method. 
For the second order method, the extreme eigenvalue is

$$
\lambda=\frac{4 \Delta x^{2} \epsilon_{0}-8 V_{0} \Delta t \Delta x \epsilon_{0}+4 V_{0} \mu \Delta t^{2} \Delta x e n_{0}}{4 \Delta x^{2}\left(\epsilon_{0}+\Delta t e \mu n_{0}\right)}
$$

which can be written as

$$
\lambda=\frac{1-2 c+\beta c^{2}}{1+\beta c}
$$

In this case, we require $c \leq 1$ for stability.

\section{A.4.2 Zero Mean Field}

In this case, we further simplify the model by assuming that the mean potential gradient is zero, and the perturbation is the entire field. While the previous system was useful for comparing treatments of the advective term, this system can be used to examine discretizations for the potential Laplacian term. Forward Euler, Backward Euler and Crank-Nicholson are compared.

This system is given by

$$
\begin{aligned}
\frac{n_{i}^{n+1}-n_{i}^{n}}{\Delta t} & =-\mu n_{0}\left\langle\frac{\partial^{2} \phi}{\partial x^{2}}\right\rangle \\
\frac{\epsilon_{0}}{e} \frac{\partial^{2} \phi_{i}^{n+1} / \partial x^{2}-\partial^{2} \phi_{i}^{n} / \partial x^{2}}{\Delta t} & =-\mu n_{0}\left\langle\frac{\partial^{2} \phi}{\partial x^{2}}\right\rangle
\end{aligned}
$$

For the Forward Euler discretization, the extreme eigenvalue is

$$
\lambda=1-c \beta
$$

For Backward Euler we have

$$
\lambda=\frac{1}{1+c \beta}
$$

For Crank-Nicholson we have

$$
\lambda=\frac{1-c \beta / 2}{1+c \beta / 2}
$$


The Forward Euler case has the severe dielectric relaxation timestep restriction. We must

use $\Delta t<2 \tau_{\text {dielectric }}$ for stability and we need $\Delta t<\tau_{\text {dielectric }}$ to prevent possible oscillations. The Backward Euler case is unconditionally stable and oscillation free. The Crank-Nicholson discretization results in an unconditionally stable method, but is not oscillation free (R-stable) unless $\Delta t<2 \tau_{\text {dielectric }}$. The concern about oscillations is that they may lead to non-linear instabilities which are neglected by the linear equations.

\section{A.5 Summary}

The electron/potential portion of the APM single grid update scheme has been linearized and the stability of this system has been analyzed. Numerical calculations show that the algorithm is stable for a large space of parameters provide the usual Courant condition is maintained $(\Delta t<$ $\Delta x / V)$. The cold electron approximation leads to another system which was investigated. Analytic formuli were developed which are useful if the cell transit time is large compared to the dielectric relaxation time (which it is for parameters of interest). In this case, the system is unconditionally stable if simple upwinding is used for the electron advection and is conditionally stable $(\Delta t<\Delta x / V)$ if second order advection is used. Finally a zero mean field system was analyzed and the effects of Forward Euler, Backward Euler and Crank-Nicholson discretizations for the potential Laplacian on the stability are discussed. This analysis illustrates the basis for the dielectric relaxation timestep constraint. 


\section{Appendix B}

\section{Derivation of the Fluid Equations}

\section{from the Boltzmann Equation}

The Boltzmann Equation involves the solution of an equation in a 7 dimensional space. An alternative approach to solving this equation is to solve a corresponding set of moment equations. These equations are obtained by multiplying the Boltzmann equation by various products of velocity components and integrating over all of velocity space. The idea behind this is that if you can satisfy these equations, your solution is likely to have the same velocity dependence as the real distribution function. For plasmas, typically 2 to 4 of these equations are solved and they have clear physical interpretations. The moments used in the model for this work, for example, correspond to the continuity, momentum and energy equations. The following derivation follows that found in [28].

\section{B.1 Velocity Distribution}

The velocity distribution is assumed to be a drifting Maxwellian. This allows the decomposition of the velocity $\vec{v}$ into a directed, drift component $\vec{u}$ and an isotropic, random thermal component $\vec{w}$. 


$$
\vec{v}(x, t)=\vec{u}(x, t)+\vec{w}(x, t)
$$

The velocity moments are defined by integrals, over all of velocity space, with the distribution function $f(\vec{v})$ as the kernal.

$$
\langle g\rangle \equiv \int g f(\vec{v}) d^{3} \vec{v}
$$

We have the following definition for the components of $\vec{u}$.

$$
u_{i}=\left\langle v_{i}\right\rangle
$$

Since the thermal component is isotropic, we have

$$
\left\langle w_{i}\right\rangle=0
$$

\section{B.2 Moments}

The basic equation from which we want to obtain moments is the magnetic field-free Kinetic Equation.

$$
\frac{\partial}{\partial t}(n f)+\frac{\partial}{\partial \vec{x}}(n f) \cdot \vec{v}+\frac{\partial}{\partial \vec{v}}(n f) \cdot\left(\frac{q}{m} \vec{E}\right)=\frac{\delta(n f)}{\delta t}
$$

Where $n=n(x, t)$ is the particle density, $f=f(x, v, t)$ is the particle probability density, and the $\delta$ symbol denotes changes due to collisional processes.

Following Golant [28], we look at the equation term by term. We want to calculate the moments for a number of velocity components, so we first calculate it for a generic moment $g$. We have for the moment corresponding to the first term.

$$
\left\langle g \frac{\partial}{\partial t}(n f)\right\rangle=\frac{\partial}{\partial t} n\langle g\rangle
$$


For the second term

$$
\left\langle g \frac{\partial}{\partial \vec{x}}(n f) \cdot \vec{v}\right\rangle=\frac{\partial}{\partial \vec{x}}(n\langle g \vec{v}\rangle)
$$

For the third term

$$
\left\langle g \frac{\partial}{\partial \vec{v}}(n f) \cdot\left(\frac{q}{m} \vec{E}\right)\right\rangle=-\frac{q}{m} n \vec{E}\left\langle\frac{\partial}{\partial \vec{v}} g\right\rangle
$$

Finally, the collisional term

$$
\left\langle g \frac{\delta(n f)}{\delta t}\right\rangle=\frac{\delta}{\delta t}(n\langle g\rangle)
$$

Putting these terms together, we arrive at the general form of the moment equations.

$$
\frac{\partial}{\partial t}\langle g\rangle+\frac{\partial}{\partial \vec{x}} n\langle g \vec{v}\rangle-\frac{q}{m} n \vec{E}\left\langle\frac{\partial}{\partial \vec{v}} g\right\rangle=\frac{\delta}{\delta t}(n\langle g\rangle)
$$

It remains to choose a representative set of values for $g$ in order to accurately represent the system. We choose to use $g=\left\{1, v_{i}, v_{i} v_{j}, v_{k} v_{k}\right\}$ which lead to the continuity, momentum and energy equations.

So, first take $\underline{g=1}$.

$$
\begin{aligned}
\langle 1\rangle & =1 \\
\left\langle v_{i}\right\rangle & =u_{i} \\
\left\langle\frac{\partial}{\partial \vec{v}} 1\right\rangle & =0
\end{aligned}
$$

Substituting into (B.6) gives the continuity equation.

$$
\frac{\partial}{\partial t} n+\frac{\partial}{\partial \vec{x}}(n \vec{u})=\frac{\delta}{\delta t} n
$$

In this case, the collisional process corresponds to an ionization source term. 
Now we look at the moment corresponding to $g=v_{i}$.

$$
\begin{aligned}
\left\langle v_{i}\right\rangle & =u_{i} \\
\left\langle v_{i} v_{j}\right\rangle & =\left\langle\left(u_{i}+w_{i}\right)\left(u_{j}+w_{j}\right)\right\rangle \\
\left\langle u_{i} w_{j}\right\rangle+\left\langle u_{j} w_{i}\right\rangle & \\
\left\langle\frac{\partial}{\partial \vec{v}} v_{i}\right\rangle & =e_{i}
\end{aligned}
$$

Before substituting these into (B.6), we can simplify the second of these quantities introducing the momentum flux tensor, the pressure tensor and the viscous stress tensor.

The momentum flux tensor is defined as follows.

$$
\Pi_{i j}=n m \int v_{i} v_{j} d \vec{v}=n m\left(\begin{array}{ccc}
\left\langle v_{1} v_{1}\right\rangle & \left\langle v_{1} v_{2}\right\rangle & \left\langle v_{1} v_{3}\right\rangle \\
\left\langle v_{2} v_{1}\right\rangle & \left\langle v_{2} v_{2}\right\rangle & \left\langle v_{2} v_{3}\right\rangle \\
\left\langle v_{3} v_{1}\right\rangle & \left\langle v_{3} v_{1}\right\rangle & \left\langle v_{3} v_{3}\right\rangle
\end{array}\right)
$$

This can also be written as

$$
\begin{aligned}
\Pi_{i j} & =n m\left\langle\left(u_{i}+w_{i}\right)\left(u_{j}+w_{j}\right)\right\rangle \\
& =n m\left[u_{i} u_{j}+\left\langle w_{i} w_{j}\right\rangle+u_{i}\left\langle w_{j}\right\rangle+u_{j}\left\langle w_{i}\right\rangle\right]
\end{aligned}
$$

The last two terms are zero due to the isotropy of $w_{i}$, so this simplifies to

$$
\begin{aligned}
\Pi_{i j} & =n m u_{i} u_{j}+n m\left\langle w_{i} w_{j}\right\rangle \\
& =n m u_{i} u_{j}+n m p_{i j}
\end{aligned}
$$

Where $p_{i j}$ is the pressure tensor. The pressure tensor can be considered to be sum of a normal scalar pressure $(p)$ and the viscous stress tensor $\tau$.

$$
p_{i j}=P \delta_{i j}+\tau_{i j}
$$


The Temperature can also be defined in terms of the thermal velocity.

$$
k T=\frac{1}{3} m\left\langle w_{k} w_{k}\right\rangle
$$

In our case, the thermal velocity is isotropic, so the viscous stress tensor is zero. Back to the moment equations, we see that we can make the substitution.

$$
\left\langle v_{i} v_{j}\right\rangle=u_{i} u_{j}+\frac{1}{n m} P \delta_{i j}
$$

Incorporating this into (B.6), we get the equation for the conservation of momentum.

$$
\frac{\partial}{\partial t}\left(n u_{i}\right)+\frac{\partial}{\partial \vec{x}}\left(n u_{i} u_{j}\right)+\frac{1}{m} \frac{\partial}{\partial \vec{x}} p_{i j}-\frac{q}{m} n E_{i}=\frac{\delta}{\delta t}\left(n u_{i}\right)
$$

In this case the collisional term corresponds to momentum robbing collisions with neutral particles.

Continuing to a moment equation for $v_{i} v_{j}$ would result in 9 additional equations. Instead, we derive an equation corresponding to the trace of this tensor. So, we take $g=v_{k} v_{k}$.

$$
\begin{aligned}
\left\langle v_{k} v_{k}\right\rangle & =\left\langle\left(u_{k}+w_{k}\right)\left(u_{k}+w_{k}\right)\right\rangle \\
& =\left\langle u_{k} u_{k}\right\rangle+\left\langle w_{k} w_{k}\right\rangle \\
& =u_{k} u_{k}+\frac{3 k T}{m} \\
\left\langle v_{k} v_{k} v_{i}\right\rangle & =\left\langle\left(u_{k}+w_{k}\right)\left(u_{k}+w_{k}\right)\left(u_{i}+w_{j}\right)\right\rangle \\
\left\langle\frac{\partial}{\partial \vec{v}} v_{k} v_{k}\right\rangle=2 u_{i} &
\end{aligned}
$$

Once again it is useful to recognize some of these terms as familiar macroscopic quantities. In this case we introduce the energy flux density and the heat flux vector.

The flux of energy transported by the particles in the i-th direction is represented by the energy flux vector. 


$$
\begin{aligned}
Q_{i} & =n \int \frac{1}{2} v_{k} v_{k} v_{i} f d \vec{v} \\
& =\frac{n m}{2}\left\langle\left(u_{i}+w_{i}\right)\left(u_{k}+w_{k}\right)\left(u_{k}+w_{k}\right)\right\rangle \\
& \left.=\frac{n m}{2}\left(u_{i} u_{k} u_{k}\right)+u_{i}\left\langle w_{k} w_{k}\right\rangle+\left\langle w_{i} w_{k} w_{k}\right\rangle+2 u_{k}\left\langle w_{i} w_{k}\right\rangle\right)
\end{aligned}
$$

With this definition we see that.

$$
\left\langle v_{i} v_{k} v_{k}\right\rangle=2 Q_{i} / n m
$$

Each of the four terms making up the energy flux vector has a clear physical interpretation.

$$
\begin{array}{llll}
\frac{n m}{2} u_{i} u_{k} u_{k} & =n u_{i} K & \Rightarrow & \text { transport of kinetic energy } \\
\frac{n m}{2} u_{i}\left\langle w_{k} w_{k}\right\rangle & =n u_{i} U \Rightarrow \text { transport of thermal energy } \\
\frac{n m}{2}\left(2 u_{k}\left\langle w_{i} w_{k}\right\rangle\right) & =u_{k} p_{i k} \Rightarrow \text { work done by the pressure force } \\
\frac{n m}{2}\left\langle w_{i} w_{k} w_{k}\right\rangle & =\vec{q} \quad \Rightarrow \text { the heat flux vector (random particle motion) }
\end{array}
$$

We can write the moment equation corresponding to conservation of energy. Noting that $U=3 k T / 2$ and multiplying (B.6) by $m / 2$ we obtain.

$$
\frac{\partial}{\partial t}[K+U]+\frac{\partial}{\partial \vec{x}}\left[u_{i}(K+U)\right]-\frac{\partial}{\partial \vec{x}}\left(p_{i k} u_{k}\right)-\frac{\partial}{\partial \vec{x}}\left(q_{i}\right)+q n E_{i}=\frac{\delta}{\delta t}(K+U)
$$

In this case the collisional processes correspond to external heating and inelastic collisions.

There is a problem with the last moment equation, however. We have assumed that the thermal velocity $w_{i}$ is isotropic. This is a good approximation, but it ignores the flow of heat due to spatial inhomogeneities in the temperature distribution. Without this anisotropy, the heat flux vector is zero. What we would like to do is to somehow include this effect without including the anisotropy in the other moment equations.

This problem is addressed in ([28]) by including a small perturbation to the assumed Maxwellian distribution.

$$
f=f_{\text {maxwellian }}\left(1-q_{k} w_{k} \frac{m}{n(k T)^{2}}\left(1-\frac{m w_{k} w_{k}}{5 k T}\right)\right)
$$




\section{Bibliography}

[1] A. S. Almgren. A Fast Adaptive Vortex Method Using Local Corrections. PhD thesis, University of California, at Berkeley, 1991.

[2] A. S. Almgren, J. B. Bell, P. Colella, and L. H. Howell. An adaptive projection method for the incompressible Euler equations. AIAA Computational Fluid Dynamics Conference, 1993.

[3] A. S. Almgren, J. B. Bell, P. Colella, and T. Marthaler. A Cartesian grid projection method for the incompressible Euler equations in complex geometries. Technical Report UCRL-JC-118091, Lawrence Livermore National Laboratory, Livermore, California, June 1994.

[4] A. S. Almgren, T. Buttke, and P. Colella. A fast adaptive vortex method in three dimensions. Journal of Computational Physics, 113(2), 1994.

[5] M. S. Barnes, T. J. Colter, and E. Elta. Large-signal time-domain modeling of low-pressure RF glow discharges. Journal of Applied Physics, 61:81, 1987.

[6] J. Bell, M. Berger, J. Saltzman, and M. Welcome. Three-dimensional adaptive mesh refinement for hyperbolic conservation laws. Siam Journal of Scientific Computing, 15(1):127-138, 1994.

[7] M. J. Berger. Adaptive mesh refinement for parallel processors. pages 182-194. SIAM Conference on Parallel Processing for Scientific Computing, 1987.

[8] M. J. Berger and S. H. Bokhari. A partitioning strategy for nonuniform problems on multiprocessors. IEEE Transactions on Computers, C-36(5):570-580, 1987. 
[9] M. J. Berger and P. Colella. Local adaptive mesh refinement for shock hydrodynamics. J. Comp. Phys., 82(1):64-84, 1989.

[10] M. J. Berger and J. Oliger. Adaptive mesh refinement for hyperbolic partial differential equations. Journal of Computational Physics, 53:484-512, 1984.

[11] A. Brandt. Multi-level adaptive solutions to boundary-value problems. Mathematics of Computation, 31(138), April 1977.

[12] J. D. Bukowski, D. B. Graves, and P. Vitello. Two-dimensional fluid model of an inductively coupled plasma with comparison to experimental spatial profiles. Journal of Applied Physics, 80, September 1996.

[13] J. D. Bukowski, R. A. Stewart, D. B. Graves, and P. Vitello. Modeling inductively coupled plasma tools with chlorine chemistry. Proceedings of the Electrochemical Society Meeting, May 1994.

[14] I. Chern and P. Colella. A conservative front tracking method for hyperbolic conservation laws. Technical Report UCRL-97200, Lawrence Livermore National Laboratory, Livermore, California, July 1987.

[15] Scott D. Cohen and Alan C. Hindmarsh. CVODE User Guide. Technical Report UCRL-MA118618, Lawrence Livermore National Laboratory, September 1994.

[16] Scott D. Cohen and Alan C. Hindmarsh. CVODE, a stiff/nonstiff ODE solver in C. Computers in Physics, 10(2):138-143, 1996.

[17] P. Colella. Multidimensional upwind methods for hyperbolic conservation laws. Journal of Computational Physics, 87:171-200, 1990.

[18] P. Colella and E. G. Puckett. Modern numerical methods for fluid flow. To be published by Cambridge University Press. 
[19] D. K. Coultas and J. H. Keller. British patent, January 1990.

[20] W. Y. Crutchfield and M. L. Welcome. Object oriented implementation of adaptive mesh refinement algorithms. Technical Report UCRL-JC-113502, Lawrence Livermore National Laboratory, Livermore, California, April 1993.

[21] G. Dahlquist and A. Bjorck. Numerical Methods. Prentice-Hall, Englewood Cliffs, New Jersey, 1974 .

[22] G. DiPeso and V. Vahedi. Analytic sheath resolution in high density plasma source simulations. 15th International Conference on the Numerical Simulation of Plasmas, September 1994.

[23] G. DiPeso, V. Vahedi, D. W. Hewett, and T. D. Rognlien. Two-dimensional self-consistent fluid simulation of radio frequency inductive sources. Journal of Vacuum Science, 12(4):1387-1396, July 1994.

[24] D. J. Economou, T. J. Bartel, R. S. Wise, and D. P. Lymberopoulos. Two-dimensional direct simulation monte carlo (DSMC) of reactive neutral and ion flow in a high density plasma reactor. IEEE Transactions on Plasma Science, 23(4):581-590, August 1995.

[25] Obtained from P. Vitello. Argon elastic scattering and ionization rates. Journal of Chemical Physics 56(12) 6068, 1972.

[26] A. Garcia. Personal communication.

[27] M. R. Gibbons. Low Frequency, Electrodynamic Simulation of Kinetic Plasmas with the DArwin Direct Implicite Particle-in-Cell (DADAPIC) Method. PhD thesis, University of California, at Davis, 1995.

[28] V. E. Golant, A. P. Zhilinsky, and I. E. Sakharov. Fundamentals of Plasma Physics. John Wiley and Sons, New York, 1980. 
[29] G. W. Hedstrom, G. H. Rodrigue, M. Berger, and J. Oliger. Adaptive Mesh Refinement for 1-Dimensional Gas Dynamics, pages 43-47. IMACS/North-Holland Publishing Company, 1983.

[30] R. Herman. Fusion - The Search for Endless Energy. Cambridge University Press, 1990.

[31] R. J. Hoekstra and M. J. Kushner. The effect of subwafer dielectrics on plasma properties in plasma etching reactors. Journal of Applied Physics, 77(8):3668-3673, April 1995.

[32] R. J. Hoekstra and M. J. Kushner. Predictions of ion energy distributions and radical fluxes in radio frequency biased inductively coupled plasma etching reactors. Journal of Applied Physics, 79(5):2275-2286, March 1996.

[33] J. D. Hoffman. NUMERICAL METHODS FOR SCIENTISTS AND ENGINEERS. McGrawHill, Inc., New York, 1992.

[34] J. Hopwood. Ion bombardment energy distributions in a radio frequency induction plasma. Applied Physics Letters, 62(9):940-942, March 1993.

[35] J. Hopwood, C. R. Guarnieri, S. J. Whitehair, and J. J. Cuomo. Electromagnetic fields in a radio-frequency induction plasma. Journal of Vacuum Science, 11(1):147-156, January 1993.

[36] L. H. Howell and V. E. Beckner. A discrete ordinates algorithm for domains with embedded boundaries. to appear in the Journal of Thermophysics and Heat Transfer.

[37] J. H. Ingold. Two-fluid theory of the positive column of a gas discharge. The Physics of Fluids, 15(1):75-85, January 1972.

[38] M. J. Kushner, W. Z. Collison, M. J. Grapperhaus, J. P. Holland, and M. S. Barnes. A three-dimensional model for inductively coupled plasma etching reactors: Azimuthal symmetry, coil properties, and comparison to experiments. Journal of Applied Physics, 80(3):1337-1344, August 1996. 
[39] R. J. LeVeque. NUMERICAL METHODS FOR CONSERVATION LAWS. BIRKHAUSER VERLAG, Basel, 1992.

[40] M. A. Lieberman and A. J. Lichtenberg. PRINCIPLES OF PLASMA DISCHARGES AND MATERIALS PROCESSING. JOHN WILEY AND SONS, New York, 1994.

[41] D. P. Lymberopoulos and D. J. Economou. Modeling and simulation of glow discharge plasma reactors. Journal of Vacuum Science and Technology, A, 4:1229-1236, 1994.

[42] D. M. Manos and D. L. Flamm. Plasma Etching: An Introduction. ACADEMIC PRESS, INC, 1989.

[43] D. F. Martin and K. L. Cartwright. Solving Poisson's equation using adaptive mesh refinement. Technical Report UCB/ERL M96/66 Electronics Research Laboratory Memorandum (unpublished). Code and documentation available at http://barkley.me.berkeley.edu/ martin/public_html/AMRPoisson.html, University of California, Berkeley, California, October 1996.

[44] J. S. Ogle. U. S. patent no. 4,948,458, August 1990.

[45] Computational Optics and Discharge Physics Group. Hybrid plasma equipment model (HPEM).

[46] R. B. Pember, P. Colella, and W. Y. Crutchfield. An adaptive Cartesian grid method for unsteady compressible flow in complex geometries. Technical Report UCRL-JC-115650, Lawrence Livermore National Laboratory, Livermore, California, November 1993.

[47] R. B. Pember, L. H. Howell, J. B. Bell, P. Colella, W. Y. Crutchfield, W. A. Fiveland, and J. P. Jesse. An adaptive projection method for the modeling of unsteady, low-mach number combustion. Western States Section of the Combustion Institute, Fall Meeting, 1997.

[48] I. Peres and M. J. Kushner. Spatial distributions of power and ion densities in rf excited remote plasma reactors. Plasma Sources Science and Technology, 5:499-509, 1996. 
[49] D. Quinlan. Amr++: A design for parallel object-oriented adaptive mesh refinement. Proceedings of the IMA Workshop on Structured Adaptive Mesh Refinement, Minneapolis, MN. March, 1997.

[50] T. J. Sommerer and M. J. Kushner. Monte carlo-fluid model of chlorine atom production in $\mathrm{Cl}_{2}, \mathrm{HCl}$, and $\mathrm{CCl}_{4}$ radio-frequency discharges for plasma etching. Journal of Vacuum Science and Technology, B, 10(5):2179-2187, September 1992.

[51] O. Steiner, M. Knolker, and M. Schussler. Solar Surface Magnetism, pages 441-470. Kluwer Academic Publishers, 1994.

[52] R. A. Stewart, P. Vitello, and D. B. Graves. Two-dimensional fluid model of high density inductively coupled plasma sources. Journal of Vacuum Science and Technology B, 12, January 1994.

[53] R. A. Stewart, P. Vitello, D. B. Graves, E. F. Jaeger, and L. A. Berry. Plasma uniformity in high-density inductively coupled plasma tools. Plasma Sources Science Technology, 4, 1995.

[54] G. Strang. On the construction and comparison of difference schemes. SIAM Journal of Numerical Analysis, 5:506-517, 1968.

[55] W. Tan, R. J. Hoekstra, and M. J. Kushner. A time dependent propagator method for long mean free path transport of neutral particles in plasma processing reactors. Journal of Applied Physics, 79(7):3423-3431, April 1996.

[56] N. Tishchenko. Personal communication.

[57] V. Vahedi. Modeling and simulation of RF discharges used for plasma processing. $\mathrm{PhD}$ thesis, University of California, at Berkeley, 1993.

[58] B. van Leer. Toward the ultimate conservative difference scheme II. monotonicity and conservation combined in a second order scheme. Journal of Computational Physics, 14:361-370, 1974. 
[59] J. VanDuzer and C. K. Birdsall. Plasma computer simulation, a bibliography for 1950-1970. Technical Report UCB/EECS QC718.4.A12 V3 1971, University of California, Berkeley, Berkeley, California, 1971.

[60] P. L. G. Ventzek, M. Grapperhaus, and M. J. Kushner. Investigation of electron source and ion flux uniformity in high plasma density inductively coupled etching tools using two-dimensional modeling. Journal of Vacuum Science Technoloty, B, 12(6):3118-3137, November 1994.

[61] P. L. G. Ventzek, R. J. Hoekstra, and M. J. Kushner. Two-dimensional modeling of high plasma density inductively coupled sources for materials processing. Journal of Vacuum Science Technology, B, 12(1):461-477, January 1994.

[62] P. Vitello. Personal communication.

[63] P. A. Vitello, R. A. Stewart, D. B. Graves, E. F. Jaeger, and L. A. Berry. Induct94: a twodimensional fluid model of high density inductively coupled plasma sources. Technical Report UCRL-MA-120465, Lawrence Livermore National Laboratory, Livermore, CA, 1995.

[64] J. VonNeumann and R. D. Richtmyer. A method for the numerical calculation of hydrodynamic shocks. Journal of Applied Physics, 21:232-237, 1950.

[65] P. Wainman, R. A. Stewart, M. A. Lieberman, D. B. Graves, and P. Vitello. Comparison of langmuir probe characterization and model predictions in a high density ICP source. Bulletin of the American Physics Society, 39(6), 1994.

[66] M. Welcome, B. Crutchfield, C. Rendleman, J. Bell, L. Howell, V. Beckner, and D. Simkins. Boxlib user's guide and manual. Software Documentation, 1994.

[67] H. Wu, B. W. Yu, M. L. Li, and Y. Yang. Two-dimensional fluid model simulation of bell jar top inductively coupled plasma. IEEE Transactions on Plasma Science, 25(1):1-6, February 1997. 
[68] D. P. Young, R. G. Melvin, M. B. Bieterman, F. T. Johnson, and S. S. Samant. A locally refined rectangular grid finite element method: Application to computational fluid dynamics and computational physics. Journal of Computational Physics, 92:1-66, 1991. 


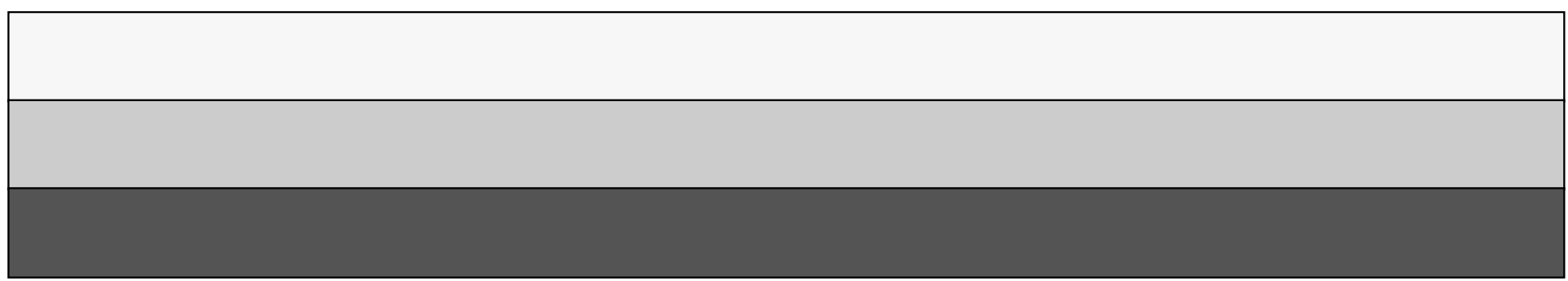

